\title{
Effects of type-I collagen fractional composition and pyridinium crosslink content on cortical bone strength in the human femur
}

Michael Blair Rice

West Virginia University

Follow this and additional works at: https://researchrepository.wvu.edu/etd

\section{Recommended Citation}

Rice, Michael Blair, "Effects of type-I collagen fractional composition and pyridinium crosslink content on cortical bone strength in the human femur" (2001). Graduate Theses, Dissertations, and Problem Reports. 1268.

https://researchrepository.wvu.edu/etd/1268

This Thesis is protected by copyright and/or related rights. It has been brought to you by the The Research Repository @ WVU with permission from the rights-holder(s). You are free to use this Thesis in any way that is permitted by the copyright and related rights legislation that applies to your use. For other uses you must obtain permission from the rights-holder(s) directly, unless additional rights are indicated by a Creative Commons license in the record and/ or on the work itself. This Thesis has been accepted for inclusion in WVU Graduate Theses, Dissertations, and Problem Reports collection by an authorized administrator of The Research Repository @ WVU. For more information, please contact research.repository@mail.wvu.edu. 


\title{
Effects of Type -I Collagen Fractional Composition and \\ Pyridinium Crosslink Content on Cortical Bone Strength in the Human Femur.
}

\author{
Michael Blair Rice \\ Thesis submitted to the Eberly College of Arts and Sciences \\ At West Virginia University \\ In partial fulfillment of the requirements for the degree of
}

\author{
Masterof Science \\ In \\ Cell and Molecular Biology \\ Phil Keeting, Ph.D., Chair \\ Ashok Bidwai, Ph.D. \\ Timothy Norman, Ph.D. \\ Department of Biology
}

Morgantown, West Virginia
2001

Keywords: Osteoporosis, Pyridinoline, Lysyl Oxidase 


\title{
Abstract \\ Effects of Type -I Collagen Fractional Composition and Pyridinium Crosslink Content on Cortical Bone Strength in the Human Femur.
}

\author{
Michael Blair Rice
}

Type-I collagen's role in the formation of bone and its inherent strength have been well documented by studies in genetic diseases such as Osteogenesis Imperfecta. Type-I collagen's role in healthy bone, and the changes that occur to collagen during aging, which may eventually lead to osteoporosis, is less understood. Changes that may occur include differences in collagen production and in its processing such as differences in glycosylation and the hydroxylation of specific amino acid residues. Changes in the reducible and non-reducible crosslink content, or changes in the ratio of crosslinks to total collagen may also occur. The present study investigated 1) type-I collagen content in cortical bone from the human femur, 2) the mature, non-reducible pyridinium crosslink content of this bone, and 3) assessed the potential relationships of these findings to fracture resistance and to the age and sex of the bone donor. It was hypothesized that higher amounts of type-I collagen and pyridinium crosslinks per dry weight of bone would correspond to increased force required for fracture.

The results indicate that the percentage of type-I collagen correlates to tension fracture resistance. Collagen content also appears to decrease with increasing age of the donor, although these data did not reach significance. A gender-based difference in collagen content of cortical bone was also observed. Pyridinium content did not correlate with either tension or shear fracture resistance in females, but a significant relationship was observed in male samples. Pyridinium content did not show a significant relationship to either age or sex, although the data suggest a larger sample size may reveal that pyridinium amount does decrease in females as they age. The ratio of pyridinium to collagen showed no relationship to fracture resistance or to age, but a gender-based difference was observed.

These studies suggest that type-I collagen content of bone is directly related to tension fracture resistance. Type-I collagen amount may also decrease during aging, and there are significant differences in the fractional composition of collagen in bone between males and females. The pyridinium content does not effect the fracture resistance except in the male sub-group; this may relate to a lower ratio of pyridinium to collagen in male cortical bone. The pyridinium amount may decrease in females as they age, although, to date only a trend toward significance could be demonstrated. 


\section{Acknowledgements}

I would like to thank Dr. Phil Keeting for the opportunity to further my education and to conduct this research. I would also like to acknowledge Dr. Keeting and Dr. Tim Norman for their input and ideas concerning this study. I would like to acknowledge Michael Kashon for his help in conducting the statistical analysis of the data. I would like to thank Pat Lutsie for her help with the computer hardware and software. Thanks to all committee members, Dr. Keeting, Dr. Norman, and Dr. Ashok Bidwai. Finally, I wish to thank all of the biology department staff for their patience and support. 


\section{Table Of Contents}

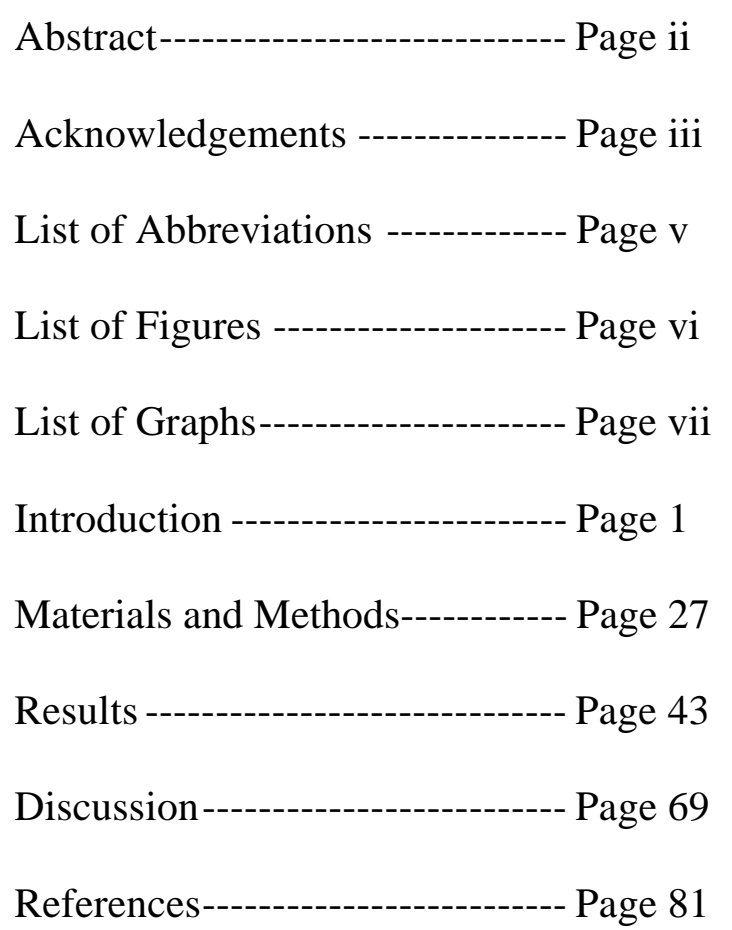




\section{List of Abbreviations}

BMD: $\quad$ Bone Mineral Density (volume)

BSA: $\quad$ Bovine Serum Albumin

DeH-HLNL: Dehydroxy-hydroxylysinonorleucine

Dpd.: $\quad$ Deoxypyridinoline

$\mathrm{Gi}_{\mathrm{c}}$ : $\quad$ Tension critical strain energy release rate

Gii $_{c}: \quad$ Shear critical strain energy release rate

GHYL: Galactosyl Hydroxylysine

HHMD: Dehydro-histidinohydroxymerodesmosine

HLKNL: Hydroxylysino-5-ketonorleucine

HPLC: $\quad$ High Performance Liquid Chromatography

Hyp: $\quad$ Hydroxyproline

NCPs: $\quad$ Non-collagenous Proteins

ODS: $\quad$ Octadecylsilane

O.I.: $\quad$ Osteogenesis Imperfecta

PITC: $\quad$ Phenylisothiocyanate

PNPP: $\quad$ P-nitrophenol-phosphate

PTC: $\quad$ Phenylthiocarbamyl

Pyr.: $\quad$ Pyridinoline

SLS: $\quad$ Segment Long Spacing crystallites

SRP: $\quad$ Signal Recognition Protein

TEA: Triethylamine 


\section{List of Figures}

Figure 1. Bone Remodeling Unit. page 4

Figure 2. Modified amino acids of type-I Collagen. page 8

Figure 3. Imino acids effect on the triple helix. page 10

$\begin{array}{lll}\text { Figure 4. Triple helix. } & \text { page } 13\end{array}$

Figure 5. Extrusion of collagen monomers. $\quad$ page 15

Figure 6. Role of lysyl oxidase. page 16

$\begin{array}{lll}\text { Figure 7. Formation of deoxypyridinoline. } & \text { page } 17\end{array}$

Figure 8. Pyr. and Dpd. and their attachment in collagen. page 19

Figure 9. Quarter-stagger array of collagen monomers. page 21

Figure 10. Hole zones and mineralization. page 24

Figure 11. Where bone slices are located on femur. page 29

Figure 12. Fracturing of bone slices. page 30

Figure 13. Formation of Phenylthiocarbamyl-hydroxyproline. page 34 


\section{List of Graphs}

Graph 1. Collagen content by location on femur. Page 48

$\begin{array}{lll}\text { Graph 2. Collagen content by tension fracture. } & \text { Page } 49\end{array}$

$\begin{array}{ll}\text { Graph 3. Collagen content by shear fracture. } & \text { Page } 50\end{array}$

Graph 4. Collagen content by age of donor. Page 51

Graph 5. Collagen content by sex of donor. Page 52

Graph 6. Pyridinium amount by tension fracture. Page 53

Graph 7-A. Pyridinium amount in males by tension fracture. Page 54

Graph 7-B. Pyridinium amount in females by tension fracture. Page 54

$\begin{array}{lll}\text { Graph 8. } & \text { Pyridinium amount by shear fracture. } 55\end{array}$

Graph 9-A. Pyridinium amount in males by shear fracture. Page 56

Graph 9-B. Pyridinium amount in females by shear fracture. Page 56

$\begin{array}{lll}\text { Graph 10. Pyridinium amount by sex of donor. } & \text { Page } 57\end{array}$

Graph 11. Pyridinium amount by location on femur. Page 58

$\begin{array}{lll}\text { Graph 12-A. Tension fracture by location on femur. } & \text { Page } 59\end{array}$

$\begin{array}{ll}\text { Graph 12-B. Shear fracture by location on femur. } & \text { Page } 59\end{array}$

Graph 13-A. Tension fracture by gender. $\quad$ Page 60

$\begin{array}{ll}\text { Graph 13-B. Shear fracture by gender. } & \text { Page } 60\end{array}$

$\begin{array}{lll}\text { Graph 14. Pyridinium amount by age of donor. } & \text { Page } 61\end{array}$

Graph 15-A. Pyridinium amount in males by age of donor. $\quad$ Page 62

Graph 15-B. Pyridinium amount in females by age of donor. Page 62

$\begin{array}{ll}\text { Graph 16. Collagen content by crystallinity. } & \text { Page } 63\end{array}$

$\begin{array}{ll}\text { Graph 17. Collagen content by } \% \text { mineral. } & \text { Page } 63\end{array}$

$\begin{array}{lll}\text { Graph 18-A. Pyridinium amount by crystallinity. } & \text { Page } 64\end{array}$

Graph 18-B. Pyridinium amount by \% mineral. $\quad$ Page 64 
Graph 19-A. Ratio (in moles) of pyridinium to collagen by tension fracture.

Page 65

Graph 19-B. Ratio (in moles) of pyridinium to collagen by shear fracture.

Page 65

Graph 20. Synergistic response of collagen and pyridinium to tension fracture.

Page 66

Graph 21. Ratio (in moles) by donor sex.

Page 67

Graph 22. Ratio (in moles) of pyridinium to collagen by donor age. Page 68 


\section{Introduction}

Osteoporosis is a disease characterized as having a low bone density and a deterioration of the microstructure of bone, leading to a decrease in bone strength and an increase in fracture risk (1-6). Factors that influence bone strength are split into two categories: material properties, and geometric properties (2,3,7-11). Material properties include collagen content, hydroxyapatite content, type and amount of non-collagenous proteins, and maturation of bone $(1,3)$. Geometric properties include bone mineral density, organization of collagen fibrils, osteon size and number, distribution of mass about the bone, and trabecular connectivity $(1-4,12,13)$.

It is generally accepted that there are two classifications of osteoporosis, referred to as Primary and Secondary Osteoporosis (3,14). Primary Osteoporosis is a condition of bone fragility found in some post-menopausal women and in some men and women as a consequence of age (3). Primary osteoporosis is further divided into two types $(14,15)$. Type- 1 is believed to be a consequence of the decrease of circulating estrogen seen in post-menopausal women, that allows an increased rate of osteoclast activity to degrade bone faster than it is replaced (2,3,7,16-22). Type-2 refers to osteoporosis involving older men and women that appears to be caused by a variety of events associated with aging, with fracture incidence exacerbated by an increased propensity to fall $(3,14,23)$. Secondary osteoporosis refers to bone loss and skeletal fragility due to disease in which the strength of bone has been compromised (3).

Fractures of the hip/femoral neck, vertebrae, and of the distal forearm (termed

Colle's fracture) account for almost $75 \%$ of all osteoporotic fractures $(6,14,24,25)$. Although 
the menopause is a primary risk factor for osteoporosis, there are additional risk factors including race, sex, and genetic predisposition, and environmental factors such as diet, attainment of peak bone mass, physical activity, and smoking $(3,11,14,15,19)$. It is estimated that roughly $40 \%$ of females of European descent over the age of fifty will eventually sustain some type of osteoporotic fracture $(1,6)$. Men over fifty and of European descent face a lifetime fracture risk of about $13 \%(1,6)$. In 1991 an estimated 7 to 10 billion dollars was spent on care for patients due to osteoporosis in the United States (14). With the increasing age of the population, this amount is sure to continue to rise.

Bone is a living tissue that protects the body's internal organs, provides sites of muscle attachment for locomotion, and serves physiological functions such as maintaining calcium and phosphate homeostasis in the body and providing a site for production of hematopoeitic tissues $(3,15,16,21,26-28)$. Bone is continuously remodeled throughout life; osteoclasts resorb bone while osteoblasts form new bone (3,11,14-16,19-22,26). This is a tightly coupled process that is essential in maintaining bone's physical and physiological functionalities $(3,19,21,29,30)$. Preosteoclasts are recruited to bone where they recognize proteins on the bone's surface. In a process not well understood, the preosteoclasts differentiate into osteoclast cells that respond to local, as well as systemic, signaling. The osteoclasts degrade old, damaged bone that is to be replaced by new bone. During degradation, signal molecules that became trapped in the bone are released and aid in the recruitment of preosteoblasts to the site. These cells then differentiate into osteoblasts to produce the new bone. As the body ages, resorption outpaces formation, the coupled interaction is no longer balanced, and bone is lost $(3,14,20,26)$. The menopause is believed to be the principle risk factor for type-I primary osteoporosis, since circulating 
estrogen, which is believed to suppress the osteoclast's activity, is no longer found at adequate levels $(3,7,16,18,22)$. Once this occurs, osteoclastic resorption of bone can further outpace osteoblastic formation of bone, which leads to a decrease in bone mass and consequently bone strength.

Osteoclasts are large multinucleated cells that are formed following the fusion of mononuclear pre-osteoclasts. In cortical bone the osteoclasts attach to the endosteal and periosteal surfaces of bone, especially in areas of bone that have been damaged. A site actively being degraded may have only one or two osteoclasts attached to the resorption site (20,27). A series of invaginations of the plasma membrane are formed where the osteoclast attaches to bone. These invaginations define the ruffled border, enclosing areas of the bone surface by the osteoclast's plasma membrane. The osteoclasts become polarized, with golgi apparati and lysozomes found between the nucleus and the ruffled border $(18,20,21)$. Lysozomal enzymes and hydrogen ions are secreted into the space between the bone and the osteoclast, causing the calcium crystals of bone to be dissolved and exposing the collagenous matrix. This is then degraded by collagenase and (possibly) cathepsins secreted by the osteoclast (18-21,27). The formation of new bone in adults only happens where the degradation of old bone has occurred; these are called remodeling sites (21) (Figure 1).

Bone is made up of an organic meshwork of proteins and an inorganic "cement" made primarily of hydroxyapatite calcium crystals. Both components are produced by the osteoblast. Of bone, $35 \%$ of its mass is the organic matrix. About $90 \%$ of this organic matrix is comprised of type-I collagen fibers, while the other $10 \%$ represents other collagen types and various non-collagenous proteins (NCP's) $(10,15,26,27,29-32)$. The 
Figure 1

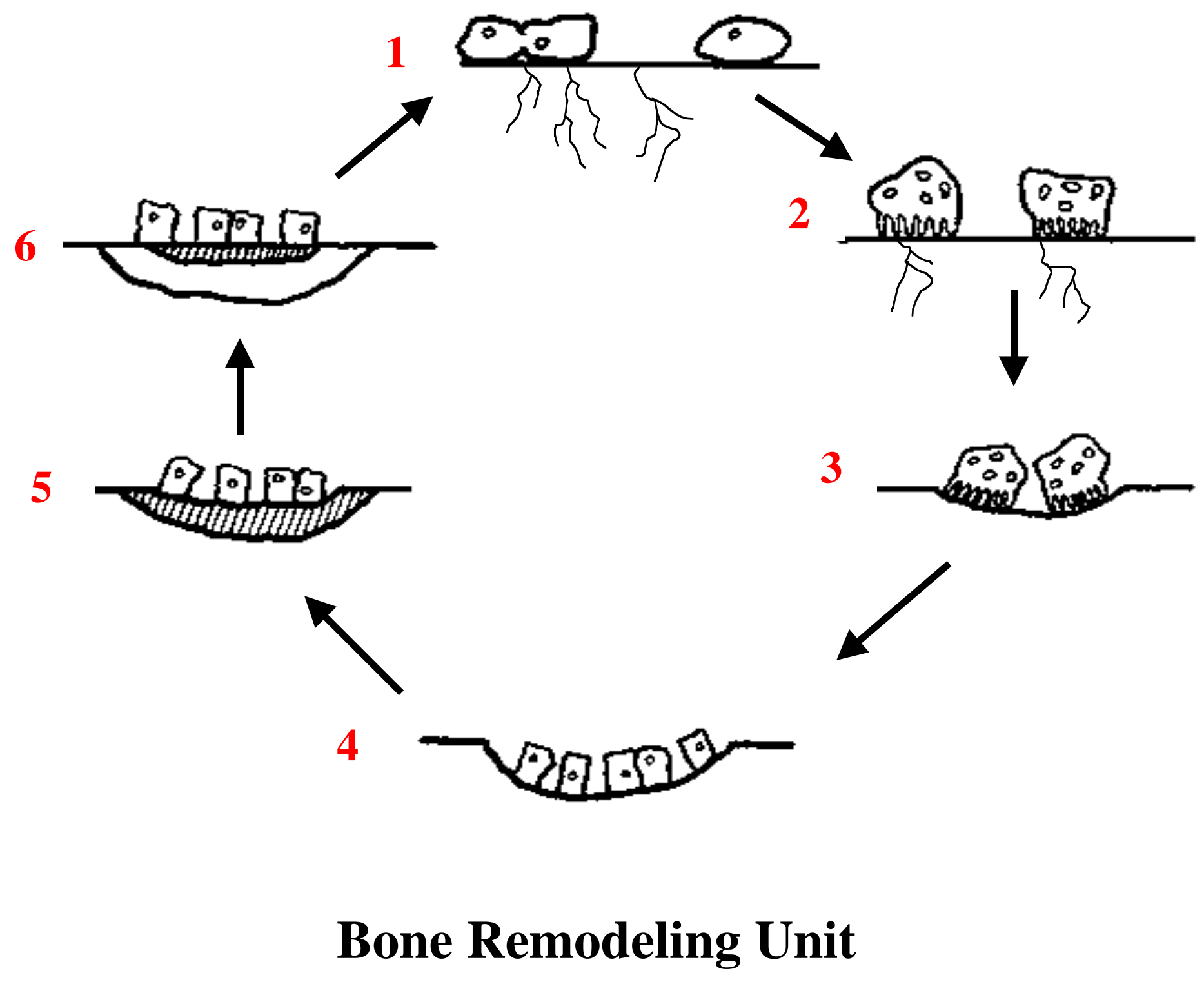

Figure 1. 1. Preosteoclasts are recruited to damaged bone where they fuse into multinucleated osteoclasts. 2. The osteoclasts form a ruffled border, effectively sealing an area of bone for degradation. 3. Osteoclasts acidify the sealed zone dissolving the hydroxyapatite, and release collagenase and other enzymes to destroy the protein matrix forming a resorption pit. 4. Preosteoblasts are recruited to the site possibly by signal molecules released during degradation. 5. Osteoblasts produce type-I collagen and noncollagenase proteins which form a new matrix, termed the osteoid. 6. The osteoid matures and becomes mineralized forming new bone, while the osteoblasts continue to produce more protein to be mineralized. 
meshwork of proteins gives bone tensile strength, allowing it to withstand the pulling forces that continuously act on bone $(5,10,28)$. The type-I collagen fibers are arranged in such a way as to produce a scaffold which becomes calcified. Mineral deposits account for about $65 \%$ of bone mass, mainly in the form of hydroxyapatite crystals $\left(\mathrm{Ca}_{10}\left(\mathrm{PO}_{4}\right)_{6}(\mathrm{OH})_{2}\right)$ (30). These crystals form parallel to the orientation of the meshwork of proteins, filling the available space and often embedding themselves into the collagen fibers. The calcium crystals give bone the strength to withstand the compression forces that are exerted on bone $(5,10,28)$. Throughout this hardened matrix, osteoblasts routinely become trapped and develop into osteocytes. Surrounding these osteocytes are fluid filled spaces known as lacunae. The osteocytes extend cellular projections that connect to other osteocytes and to osteoblasts on the surface of the bone; these projections are surrounded by fluid filled channels called canaliculae. The osteocytes may aid in maintaining mineral homeostasis or act as mechano-sensitive cells $(21,30)$. The network of lacunae and canaliculi is extensive, with an estimated surface area of $1000-5000 \mathrm{~mm}^{2}$ in an adult human skeleton. Approximately 1.0 - 1.5 liters of bone extracellular fluid (ECF) fill these periosteocytic spaces (27).

Collagen is the major constituent of the organic matrix in bone, and accounts for over $90 \%$ of the protein content. Several isotypes of collagens are found in bone,

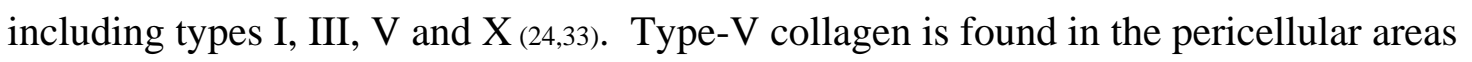
around the osteocytes, and in the canaliculi that surrounds the osteocyte's cellular projections, but it is not found in the scaffolding of the bone's extracellular matrix (24). In hyaline cartilage produced by chondrocytes that becomes mineralized, collagen type-X has been found to be produced immediately before calcification (33). Of all the collagen 
types, type-I collagen is the predominant form found in bone. Synthesis of the type-I procollagen sub-unit occurs in the osteoblast, where the protein goes through several cotranslational and post-translational modifications before folding into its tertiary structure, the triple helix. After the procollagen molecule is extruded from the cell and is cleaved of its terminal extensions, the individual triple helical collagen molecules go through a structured assembly into five-stranded units that form microfibrils. These microfibrils assemble into fibrils, which subsequently become organized as collagen fibers. These collagen fibers are arranged either into orientated layers on the bone, called lamella, or as loose bundles as is seen in woven bone.

Prior to the mineralization of the newly formed collagen matrix of the osteoid is a 5 to 10 day period of time during which the matrix matures. There are several theories as to what occurs during maturation, including the crosslinking of collagen fibers, the removal of a mineralization inhibitor, or the complex formation between collagenous and non-collagenous proteins. Following the maturation of the osteoid, hydroxyapatite crystal seeds are deposited, which grow to produce the new mineralized bone $(27,30,34)$.

The type-I procollagen molecule is made up of three sub-units, two alpha-1 subunits $(\alpha 1(\mathrm{I}))$ and one alpha-2 sub-unit $(\alpha 2(\mathrm{I}))$, together assembling into the triple helix. The genes for the $\alpha 1(\mathrm{I})$ sub-unit and the $\alpha 2$ (I) sub-unit are found on chromosomes 17 and 7 , respectively $(31,36,37)$. Of the fiber-forming collagens, all have similar genetic sequences within the exons that code for the amino acids of the triple helix-forming region of the proteins (33). An ancestral 54 base pair exon appears to have duplicated itself several times within the genes, as exons of 54 or 108 base pairs, are remarkably conserved throughout various species, and make up the lengthy triple helix region of the 
proteins (26,36,37). The conservation of the sequence of the 18 amino acid repeats specified by the 54 base pair repeats is critical to the structure of type-I collagen, and even a single point mutation within the repeat can have severe to lethal consequences $(33,36-42,47)$. Defining the helical domain of the procollagen's sub-units is the amino acid repeat of glycine-X-Y, where $\mathrm{X}$ is usually a proline (Pro), and $\mathrm{Y}$ is often hydroxyproline (Hyp) $(26,36,37,41)$. Thirty-three percent of type-I collagen's amino acids are glycine while another $15-30 \%$ begins as proline; the repeat of Gly-Pro-Hyp represents about $10 \%$ of each of the protein sub-unit's sequence $(33,36,43,44)$.

Translation of collagen's mRNA occurs on the rough endoplasmic reticulum $(32,33,36)$. Both of the procollagen chains $\alpha 1(\mathrm{I})$ and $\alpha 2(\mathrm{I})$ are roughly 160,000 Daltons in size. Both of the procollagen sub-units consist of a central helical domain (100,000 Da), a non-helical propeptide extension on the amino end $(25,000 \mathrm{Da})$ that includes a leader sequence, and a non-helical propeptide extension on the carboxy end $(35,000 \mathrm{Da})$ that contain cysteine residues for disulfide bonds that help control the folding of the collagen sub-units into their triple helix form $(26,31,33)$. The leader sequence is a short stretch of predominately hydrophobic amino acids that are recognized by a signal recognition particle (SRP) located on the surface of the rough ER. The SRP acts as a receptor and guides the protein being formed into the membranous rER where co-translational and post-translational processing occur (43).

During, and immediately after, translation of the procollagen sub-units, several enzymatic modifications of the protein occur. While translation is occurring, specific newly incorporated proline and lysine residues are recognized as hydroxylation 


\section{Figure 2.}
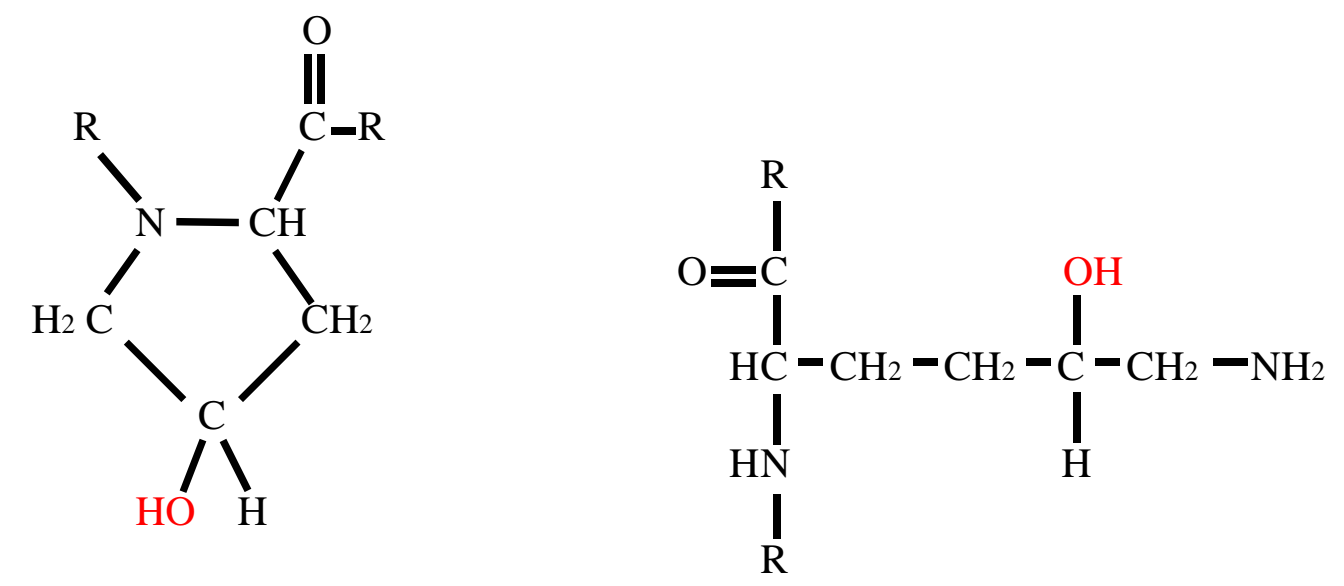

4-Hydroxyproline

5-Hydroxylysine

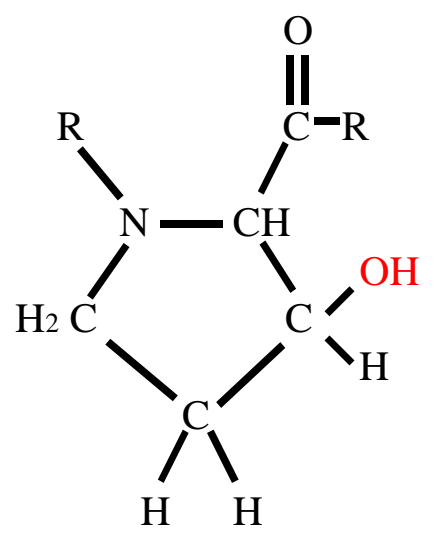

3-Hydroxyproline

Figure 2. The three modified amino acids that are found in type-I collagen. Both proline and hydroxyproline residues help form, and strengthen the triple helix while lysine and hydroxylysine residues may become glycosylated or form crosslinks between collagen monomers. 
candidates (26,36) (Figure 2). If the amino acid at the Y position is lysine, it can be hydroxylated to form 5-hydroxylysine and if the amino acid is proline, it can be hydroxylated to form 4-hydroxyproline. A few proline residues at the $\mathrm{X}$ position are hydroxylated forming 3-hydroxyproline, but this occurs only if the Y proline has been hydroxylated previously (36). Hydroxyproline (Hyp) is found in abundance in all collagen types and has a ratio of $0.14 \mu \mathrm{g} \mathrm{Hyp} / 1.0 \mu \mathrm{g}$ of type-I collagen (45). The hydroxylation of prolines and lysines are catalyzed by several types of prolyl- and lysyl-hydroxylases, respectively $(33,43,46)$. These hydroxylations are completed prior to triple helix formation and are required for the sub-units to fold into the triple helix; it appears that the formation of the triple helix may limit the amount of hydroxylation that occurs $(38,40,47,48)$. The importance of proline and hydroxyproline, which are more correctly identified as imino acids, is that their chemical structures help form and stabilize the helical structure of collagen. This occurs in two ways. The imino acids do not allow rotation between the nitrogen and the $\alpha$ carbon which aids in formation, and the ring structure of the residues confers rigidity and inflexibility, which limits the bending of the triple helix (41,44) (Figure 3). Hydroxyproline residues also may strengthen the triple helix by forming intramolecular hydrogen bounds involving water bridges $(40,43,44)$. An example of the functional importance of these modifications is shown when collagen is formed in the absence of an active prolyl hydroxylase. These resultant incompletely processed collagen molecules denature at $24^{\circ} \mathrm{C}$, while normal collagen denatures at $39^{\circ} \mathrm{C}(43)$. The hydroxylysine residues do not appear to contribute to the stability of the triple helix. However, hydroxylysine residues contribute to the stability on the collagen fiber as a 
Figure 3.

Glycine Hydroxyproline Hydroxylysine Glycine Proline

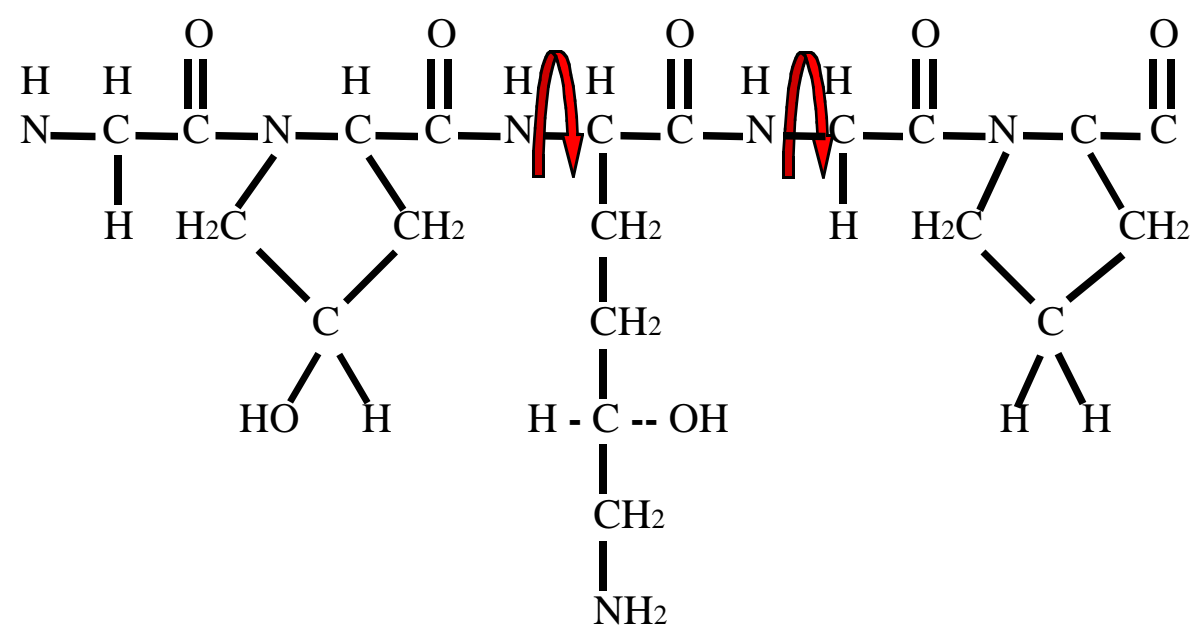

Figure 3. Imino acids in the primary structure of type-I collagen. The ring structure of the imino acids do not allow for rotation between the nitrogen and the $\alpha$ carbon of the imino acid's backbone as illustrated by the arrows circling the typical N-C bond. These rings also help stabilize the tertiary structure of the protein, the triple helix, by limiting the amount of bending of the collagen monomer. 
whole, because hydroxylysine and lysine residues are used to form intramolecular and intermolecular covalent crosslinks $(12,42,43,47,48)$. After the hydroxylation of certain lysine residues, carbohydrates become covalently bound to some of the hydroxylysine residues by galactosyltransferase and glucosyltransferase action $(26,33,36,41,49)$. The carbohydrates added are the monosaccharide galactose, and galactose-glucose disaccharides (33). The function of these sugars is not well understood but they may be involved with fibril assembly outside of the osteoblast, crosslinking of monomers, or the mineralization of the matrix. Their importance is shown by an increase in the amount of galactosylhydroxylysine in urine (GHYL) that has been reported to correspond to an increase in fractures (5). Another carbohydrate side chain is found in the C-terminal propeptide, attached to an asparagine residue. This carbohydrate side chain is composed of Nacetylglucosamine and mannose and may aid in the alignment of the sub-units prior to formation of the triple helix (26). These covalent modifications occur during translation of the mRNA, while the protein subunit still remains attached to the ribosome. Removal of the $\mathrm{N}$-terminal leader sequence precedes release of the polypeptide from the ribosome. After their release, while still within the ER, the three procollagen sub-units align so that intrachain and interchain disulfide bonds are formed between cysteine residues found in the C-terminal propeptide extensions $(26,33,38,41,43)$. The proper alignment of the $\mathrm{C}$-terminal propeptide extensions, and the amino acid composition of each sub-unit, provides for the spontaneous formation of the triple helix $(26,31,33,38,41)$. The amino propeptide has intrachain, but no interchain, disulfide bonds (36). Found in the triple helical region of each chain is the Gly-X-Y repeat. The triple helix has so little room within its interior that glycine's R-group, hydrogen, is the only one small enough to fit inside the triple 
helix (43). Due to this constraint the sub-units of the helix must be staggered so that glycines' R-groups from each of the sub-units face the interior. This causes the glycine from one sub-unit to align opposite to the $\mathrm{X}$ from another sub-unit, and a hydrogen bond is formed from the $\mathrm{N}-\mathrm{H}$ of glycine and the carbonyl oxygen of the $\mathrm{X}$ residue $(40,43)$. It has been proposed that the hydroxyproline residues may form interchain hydrogen bonds. Intrachain hydrogen bonding involving water bridges and hydroxyproline are believed to be formed. The number of hydrogen bonds for each Gly-X-Y repeat remains unclear $(33,40)$.

The way in which the helix is folded also strengthens the fiber as a whole. The individual sub-units have a left-handed coil with approximately 3.3 residues per turn and 8.7 A between glycine residues (33,36,43). The sub-units in the collagen triple helix structure have a rise per residue of $2.9 \AA$ as compared to the $1.5 \AA$ rise per residue for the alpha helix, a common secondary structure of many proteins $(33,43,44)$. This large rise per residue probably does not allow for intrachain hydrogen bonds to be formed directly between residues (as is seen in the alpha helix), but intrachain hydrogen bonds may be formed involving water bridges (33). The three left-handed helical sub-units form a righthanded tertiary structure, the triple helix $(36,40,43,44)$. The opposing twists of the individual sub-units and the triple helix insure that collagen can withstand tension without pulling out the twists (43) (Figure 4).

After the folding of the procollagen sub-units, the soluble procollagen molecule is transported to the golgi apparatus via microsomal lumen (38). In the golgi, mannose-rich carbohydrate extensions are added to the molecule, which is then packed into vesicles 
Figure 4

\section{The Triple Helix}

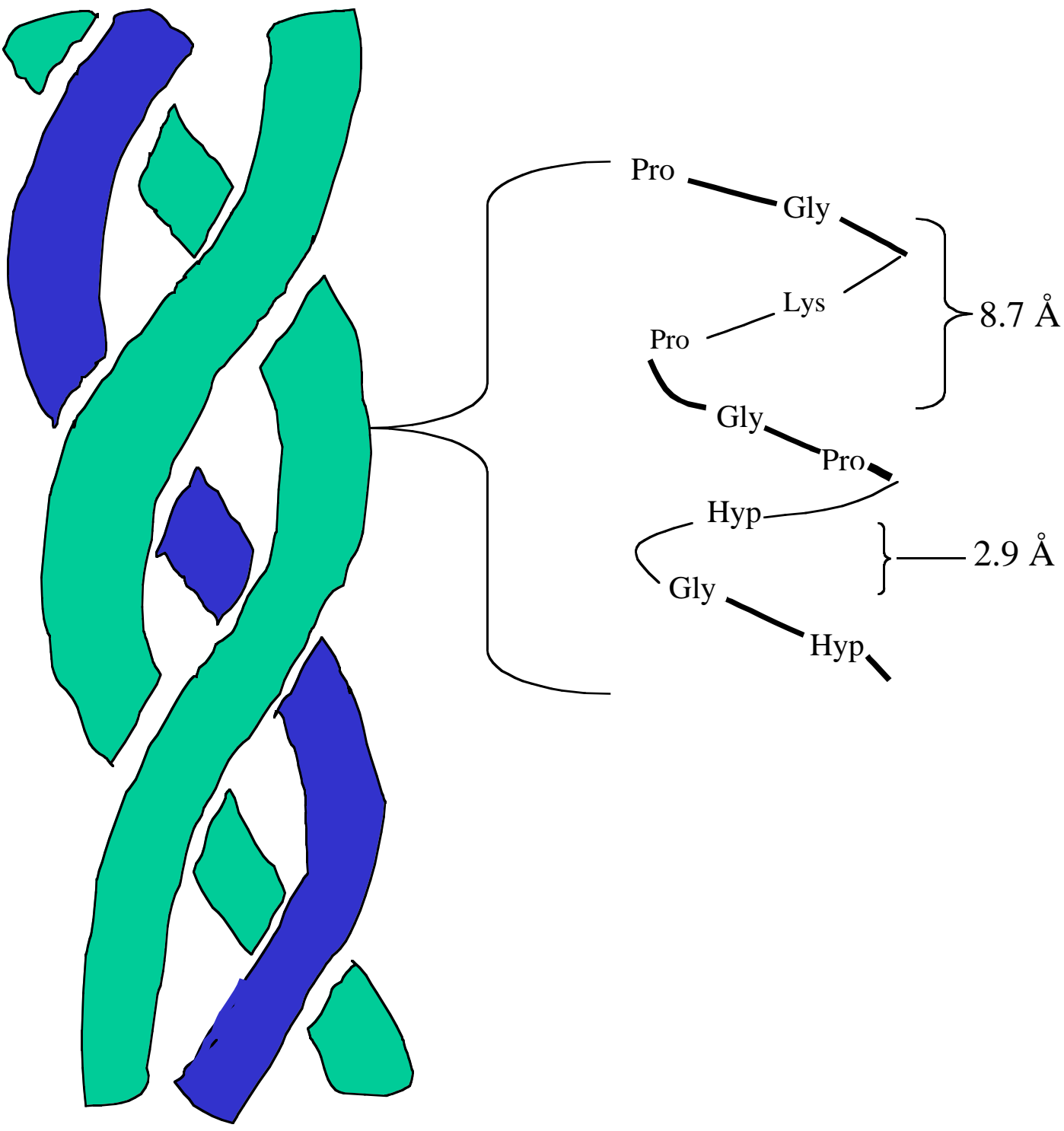

Figure 4. Collagen's tertiary structure is formed from three left-handed coiled subunits that form a right-handed supercoil, termed the triple helix. On the individual subunits each amino acid residue is $2.9 \AA$ apart with 3.3 residues completing a full turn. This large rise per residue does not allow intrachain hydrogen bonding to occur except (possibly) by water bridges. In the interior of the triple helix interchain hydrogen bonding occurs between glycine's nitrogen-hydrogen and the carbonyl oxygen form another sub-units " $\mathrm{X}$ " residue. 
for secretion from the cell $(32,33)$. In the vesicles, the resultant tropocollagen molecules condense laterally into what is called Segment Long Spacing (SLS) crystallites (33,51). Fibrillogenesis does not occur in the vesicles possibly due to the non-helical propeptide extensions (31,33). As the vesicles exit the cell, aminopeptidases cleave the aminopropeptide extension, and then carboxypeptidases cleave the C-terminal propeptide extension $(26,33,36,43)$. Both the amino and the carboxy propeptides are found within the bone matrix in a phosphorylated form, and may have a function in remodeling, along with other trapped proteins, upon release by bone degradation $(16,21,26,52,53)$. Tropocollagen's solubility is immensely decreased once trimmed of the non-helical ends, and this may aid in the spontaneous fibrillogenesis of the collagen monomers (41) (Figure 5). Once out of the cell and trimmed of its non-helical ends, the molecule is considered a collagen monomer.

In the extracellular environment the newly trimmed collagen monomers are acted on by the enzyme lysyl oxidase, an extracellular amine oxidase $(33,36,54,55)$. Lysyl oxidase converts some of the lysine and hydroxylysine residues into aldehydes by catalyzing the oxidative deamination of the amino acid's R-group (33,56,57) (Figure 6). The enzyme probably uses molecular oxygen as a co-substrate and a hydrogen acceptor (33). The enzyme is believed to act on collagen monomers or aggregates of microfibrils because once fiber formation occurs, the enzyme, which is $30,000 \mathrm{Da}$ or multiples thereof, would not be able to reach into the interior of the fiber (33). Several types of crosslinks are formed; reducible types include hydroxylysino-5-ketonorleucine (HLKNL), dehydrohydroxylysinonorleucine (DeH-HLNL), and dehydro-histidinohydroxymero desmosine 


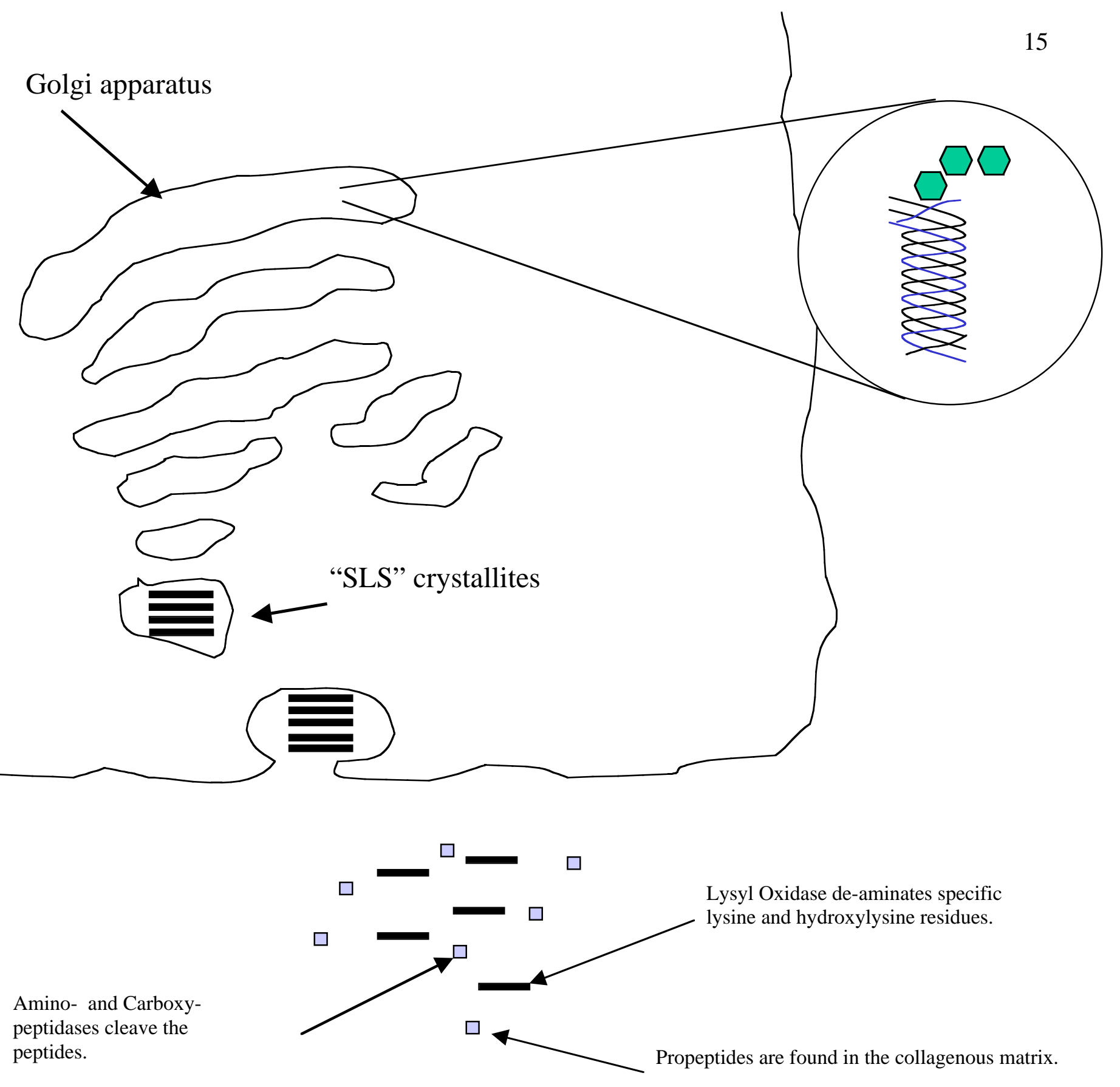

Amino- and Carboxypeptidases cleave the

Figure 5. The triple helix passes through the Golgi apparatus where it is further modified. From the Golgi, the tropocollagen molecules are packed into secretory vesicles for extrusion from the cell. Inside the vesicles the tropocollagen molecules form segment long spacing "SLS" crystallites. Upon extrusion from the cell, amino and carboxy peptidases cleave the propeptides and the resultant collagen molecules are acted on by lysyl oxidase and can now form the 5-stranded unit. 
Figure 6. Oxidative deamination of hydroxylysine by lysyl oxidase. The resulting aldehyde can spontaneously react with another hydroxylysine to form a bivalent crosslink termed Lysinonorleucine. This reacts with another lysine or hydroxylysine to form the trivalent non-reducible pyridinium crosslinks.

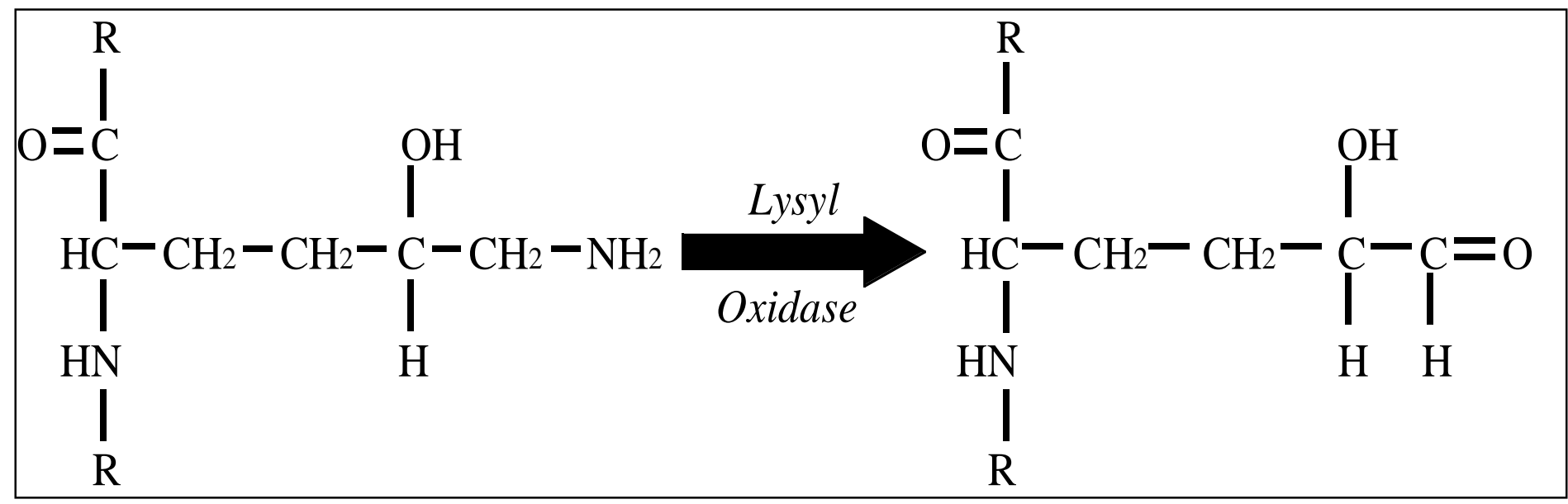

(HHMD), and the non-reducible pyridinium crosslinks, pyridinoline and deoxypyridinoline. The DeH-HLNL and the HHMD are lysine-aldehyde derived crosslinks, and have been detected in early stages of the bone collagen matrix formation. As the matrix matures and mineralization begins, the hydroxylysine-aldehyde derived crosslinks, pyridinoline and deoxypyridinoline, predominate $(48,57,66)$. Several studies have found an increase in lysine hydroxylation in osteoporotic bone, yet do not agree whether the increase in hydroxylysine is on the $\alpha 2$ (I) chain or the $\alpha 1$ (I) chain, or both $(5,12,48,58)$. The increase in hydroxylysine did not cause a change in pyridinium crosslink content in osteoporotic bone, but a decrease in HLKNL was noted (48). Also, an increase in hydroxylysine content in collagen has been correlated to a decrease in trabecular bone volume.

From the aldehyde derivatives of the hydroxylysine residues, two types of nonreducible trivalent crosslinks are formed, 3-hydroxylysylpyridinoline, formed from three hydroxylysine residues, and lysylpyridinoline, formed from two hydroxylysines and one 
Figure 7.<smiles>[R]NC(CCC([2H])C=O)C([R])=O</smiles>

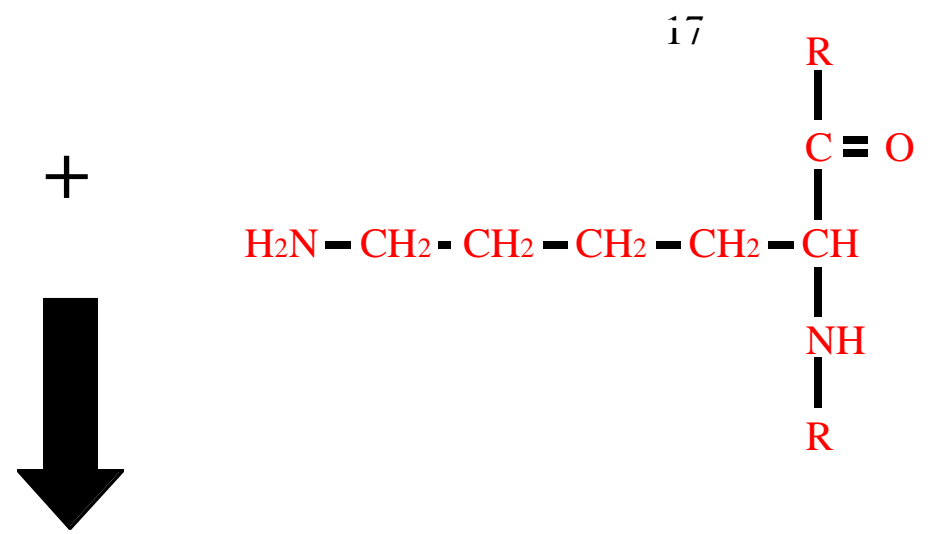

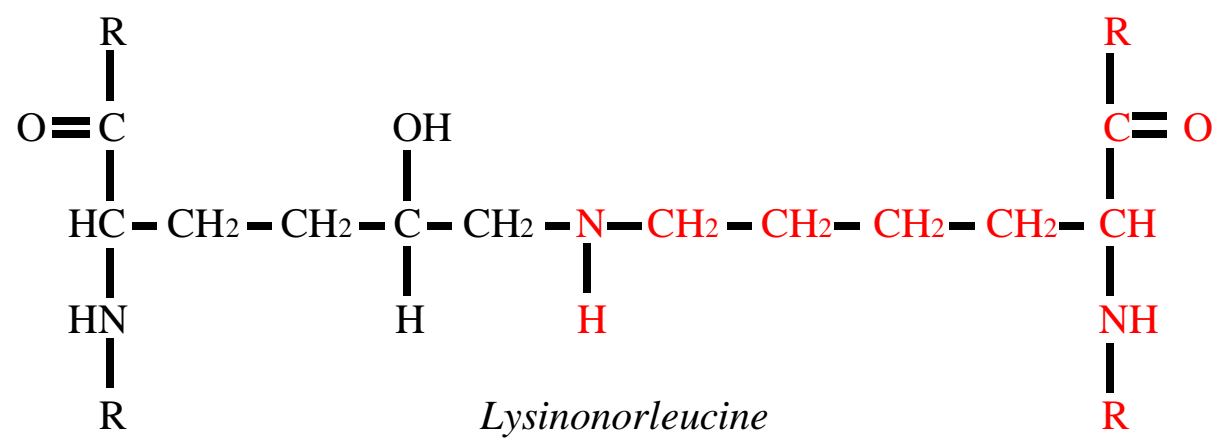<smiles>[R]NC(CCCCn1cc(O)c(CC(N[R])C([R])=O)c1CCC(N[R])C([R])=O)C([R])=O</smiles>

Formation of deoxypyridinoline from two hydroxylysines and one lysine. 
lysine residue $(12,26,33,56,57)$ (Figure 7). These crosslinks are commonly termed pyridinolines and deoxypyridinolines, respectively. These non-reducible crosslinks connect three collagen monomers together and help to stabilize the fibril $(26,31,59,62)$. The crosslinks are found at two sites in the helical region of the collagen molecule. One crosslink has residues in the aminotelopeptide regions of two molecules that form a covalent bond at or near residue 930 in the helical domain of the third monomer. The second crosslink has residues in the carboxytelopeptide regions of two molecules linked to residue 87 in the helical domain of the third molecule $(42,50)$ (Figure 8$)$. The telopeptide regions have been shown to be involved in the proper nucleation and fibril elongation, in vitro (60). This nucleation and fibril formation may involve crosslinks properly aligning the collagen monomers. During bone turnover and collagen breakdown, the pyridinolines are excreted as free and peptide bound crosslinks that can be monitored along with other markers to predict bone turnover that may indicate fracture risk $(5,15,16,19,26,31,33,53-57,59,61,62)$. Pyridinoline appears to be ubiquitous within the body's collagen, while the deoxypyridinoline crosslink is found primarily in mineralized tissues, such as dentin and bone $(15,19,55-57,59,62,63)$. It is estimated that $22 \%$ of the crosslinks in bone are deoxypyridinoline, and the combined concentration of crosslinks is estimated to be 0.3 moles / 1.0 mole of type- 1 collagen $(26,57,63$,$) . It is estimated that for every three$ type-I collagen monomers there is one pyridinium crosslink. As the crosslinks are being formed, fibrillogenesis is also occurring. The five-stranded microfibril aggregate appears to be the next structure formed. This five stranded structure proposed by Smith (64), satisfies the quarter staggered theory (discussed below), and X-ray microscopy appears to 
support this structure $(33,43,44,51,64,65)$. In non-mineralized bone, each monomer is $300 \mathrm{~nm}$ long, and when one is laid down next to another monomer it overlaps it by 3/4 of its length, leaving about $68 \mathrm{~nm}$ non-overlapped at each end. The placement of three more monomers along side the first two, each at a quarter stagger, forms one unit, with each monomer $68 \mathrm{~nm}$ displaced from the next, and the tail of the first monomer spaced about $40 \mathrm{~nm}$ from the head of the fifth. This $40 \mathrm{~nm}$ "hole" region, where only four of the five monomers overlap, is rich in carbohydrates attached to hydroxylysine residues, and these sugars may play a role in forming this pre-fibril unit, and later during mineralization $(43,44,49)$. One aspect under scrutiny questions if charges along the length of the collagen monomers aid in the staggering of the monomers themselves $(33,44)$. Stacking unit upon unit forms a single fibril, which along side thousands of other fibrils, forms the tough collagen fibers. Between fibrils crosslinking also occurs, further strengthening the fiber (Figure 9).

In the normal production of bone, the orientation of the collagen fibers produced by the osteoblast is parallel in each layer of collagen, or lamella. Between lamella, the orientation of the fibers occurs at angles to increase the amount of collagen per volume of bone (27). The lamellae are laid in sheets on top of each other as seen on the flat surfaces of bone, such as the periosteum, or deposited in concentric circles, such as around a Haversian canal or canaliculi (27). Several studies have indicated that collagen fiber orientation, in response to load bearing, play a significant role in bone strength $(10,11)$. Instances occur where the fibers are produced rapidly and deposited in unorganized bundles called woven bone. Woven bone is made at times of fracture healing, bone 


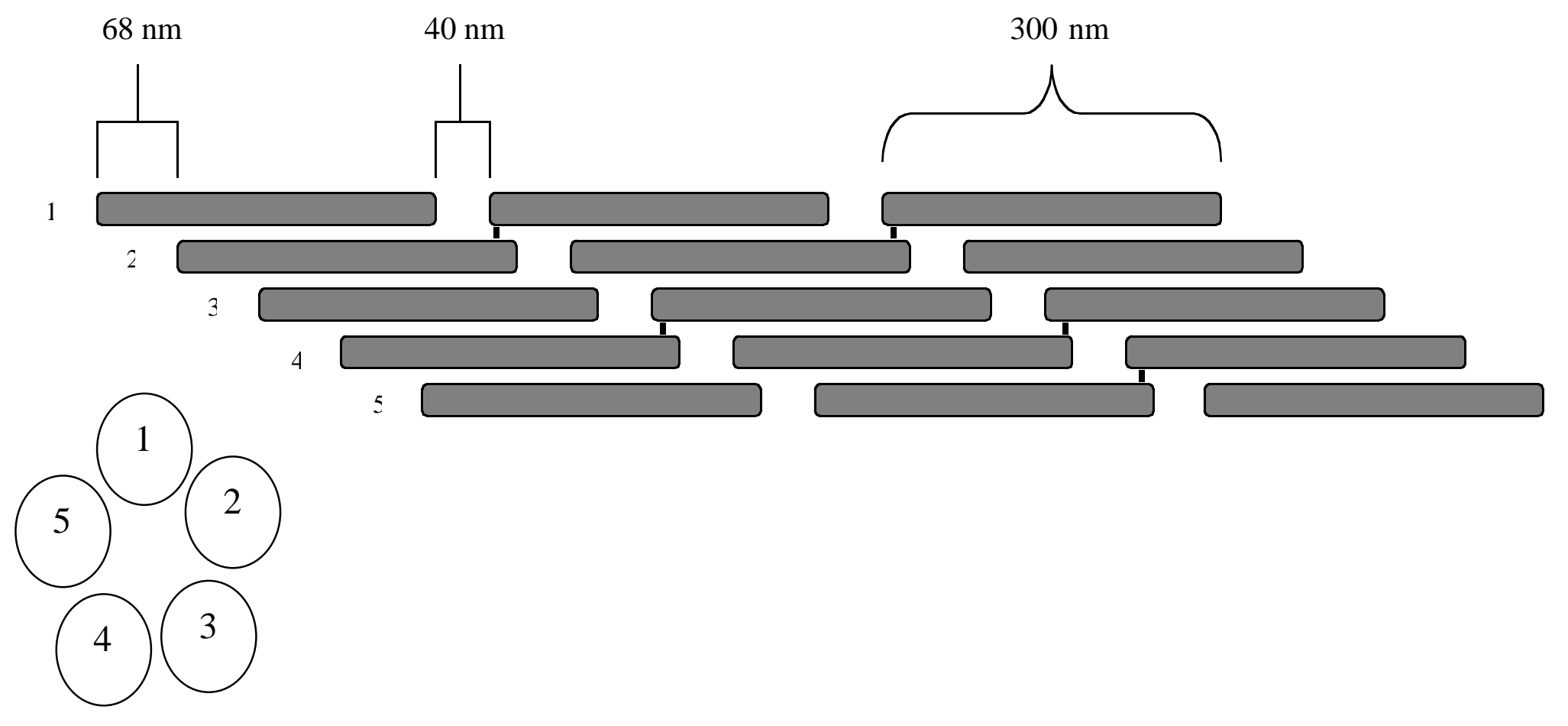

Figure 9. Each collagen monomer is displaced by about one quarter of it's length along the side of another monomer. Five monomers "quarter-staggered" together form a 5stranded microfibril that when stacked upon each other leave a $40 \mathrm{~nm}$ "hole" between the carboxy end of one monomer and the amino end of the next. This hole regions are rich in carbohydrates and sites of mineral deposition 
elongation of children, and during specific metabolic diseases (27,52). In fracture healing, the woven bone is eventually remodeled and mature lamellar bone is deposited. During bone growth in children, the woven bone allows space for hematopoeitic bone marrow to form. Soon after the formation of this bone marrow, the woven bone is remodeled and replaced with mature lamellar bone.

Mineralization of the matrix is also a function of the osteoblast, and occurs only after matrix maturation. Osteoid maturation and mineralization occurs over a period of about 5 to 10 days in humans $(27,30,66)$. The maturation of the matrix is not well understood, fibrils fuse into collagen fibers, non-collagenous proteins interact with the matrix, while the surrounding microenvironment becomes conducive to mineralization; however, the signals for mineralization to begin have not yet been identified. The signal may include the chemical crosslinking of the collagen microfibrils, the removal of a mineralization inhibitor, or the synthesis and addition of non-collagenous proteins, such as osteocalcin or osteonectin, to the matrix $(12,21,30,34,42,65-69,71)$. Of the non-collagenous proteins, osteocalcin and osteonectin are considered probable participants in the mineralization process. Osteocalcin contains gamma-carboxyglutamic acid residues, whose two negative charges can bind a calcium ion, though it remains unresolved as to how much osteocalcin the human bone contains $(15,21,26,29,52,68)$. Also, the bones of warfarin treated animals, which blocks the $\gamma$-carboxylation of glutamic acid in osteocalcin, appear to be normal in all aspects with the exception of decreased levels of osteocalcin retained in bone $(29,52)$. Warfarin treated rats also show a decrease in osteoclast recruitment and bone resorption (29). Osteocalcin knockout mice show a decrease in crystal size but an increase in mineral content in their bone (84). This suggests 
that osteocalcin may be involved in resorption-formation coupling and that its presence in bone may be due to the hydroxyapatite to which it binds. Osteonectin is believed to be involved, in part due to its high affinity for both hydroxyapatite and calcium ions and for collagen $(29,31,69)$. The removal of a mineralization inhibitor, such as pyrophosphate or the glycoprotein decorin, may play a part. Decorin has been shown to be in the uncalcified osteoid, but not in areas that are mineralized (34).

There are two pathways by which the mineralization of bone can occur, vesicular mineralization and non-vesicular mineralization $(21,31)$. In both processes, the calcium crystals grow and elongate parallel to the collagen fibers $(11,34)$. In calcified cartilage and in woven bone, the osteoblast produces membrane bound vesicles that exocytose from the plasma membrane, migrate to the matrix, and mineralize from the inside out. As the enclosed hydroxyapatite crystal grows, it ruptures the vesicle, and continues to accumulate in size until inhibited $(31,34)$. Mineralization of the crystals appears to be inhibited by pyrophosphate, adenosine triphosphate (ATP), and by acidic noncollagenous proteins that are also produced by the osteoblast (31).

In lamellar bone, which has the collagen fibers laid at angles on top of each other, the mineralization does not use vesicles, probably due to space restrictions (31). Here the mineralization occurs between the collagen fibers and at junctions of collagen and noncollagenous proteins. The mineralization occurs within fibers and expands around them, oftentimes distorting the collagen fibers (65). The $40 \mathrm{~nm}$ "hole" region is also an area of mineralization, due to the fact that there is available space and possibly due to the high amount of carbohydrates found covalently attached to the hydroxylysine residues $(10,31,51)$. The "holes" are thought to form continuous channels that allow for a seam of mineral to 

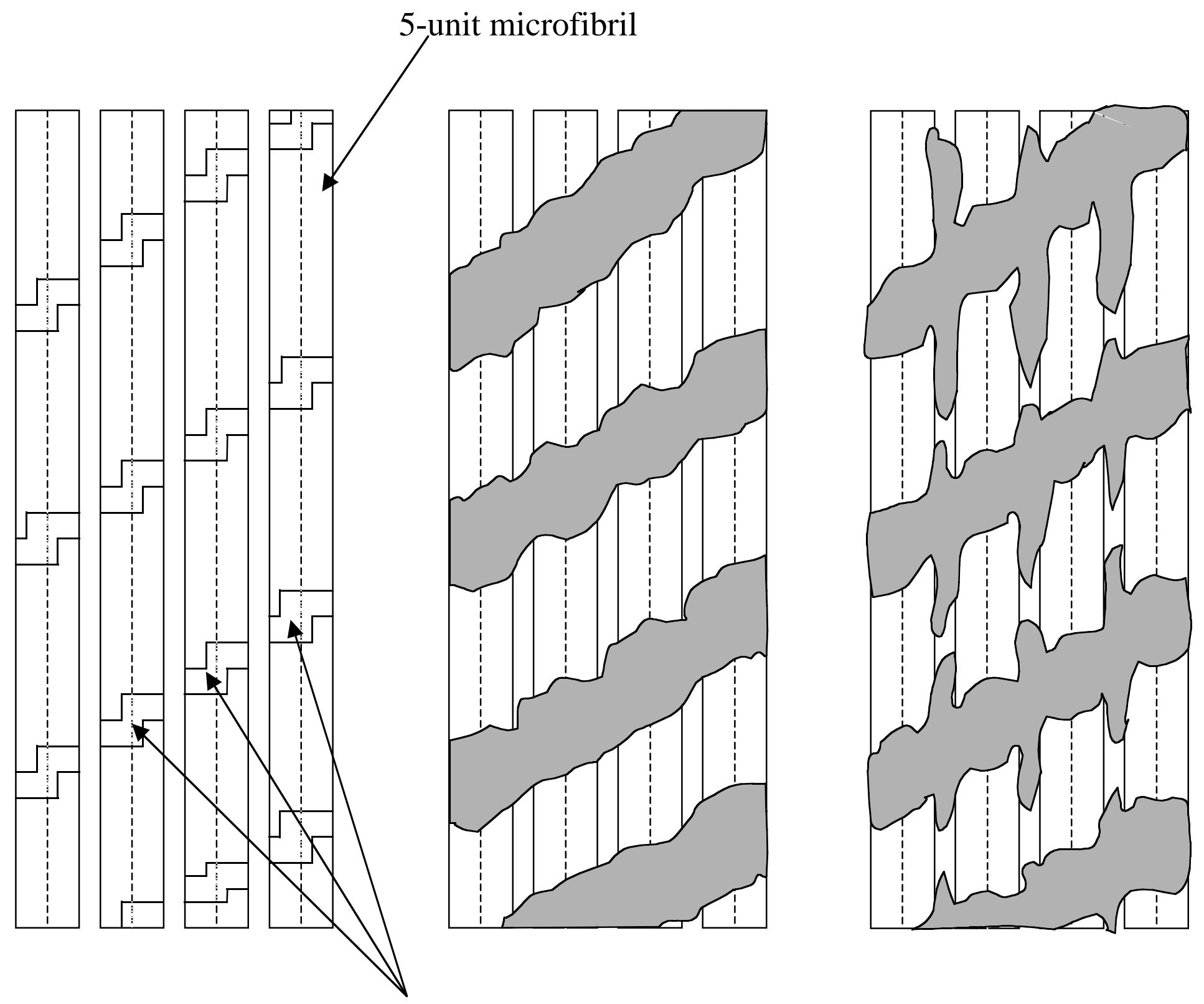

Formation of continuous channel by "hole" zones

Figure 10. The hole zones of microfibrils align to form seams where mineral crystals are deposited and grow. The crystals enlarge and push against the fibrils, distorting them and sometimes embedding themselves into the fibrils. What initiates crystal deposition and growth are unknown. 
be deposited and grow (28,70). (Figure 10) The stimulus for mineralization in lamellar bone is not known, but non-collagenous proteins probably play a role since purified collagen is a poor initiator of mineralization. This may be why collagen fibrils band together to form fibers prior to mineralization. Acidic non-collagenous proteins appear to inhibit mineralization of lamellar bone, but available space must also a contributing factor $(31,34)$. Interestingly, osteonectin, one of the non-collagenous proteins thought to be involved in mineralization, may also be involved in the inhibition of mineralization. When bound to collagen, osteonectin promotes the deposition of hydroxyapatite onto the scaffold surface, but when in solution, osteonectin saturates mineralization sites and prevents further mineralization possibly by competing with bone for calcium ions (52). This scenario is also being investigated for other proteins involved in bone, including osteocalcin and phosphoproteins (67,71).

The aim of the present study is to assess which biochemical and morphological characteristics of bone are determining factors for bone strength. Morphological features include osteon size and number, bone density, and microdamage to bone, while hydroxyapatite crystallinity, mineral content, collagen content, osteocalcin content and pyridinium crosslink content are all biochemical features. Some of these issues have been evaluated in companion studies done by collaborators (72). The work described within this thesis is focused on the analysis of type-I collagen fractional content and on the amount of pyridinium crosslinking found in the collagen. Quantification of type-I collagen, and the pyridinium markers, are then correlated to both tension and shear fracture resistance. This is done in an attempt to identify the most important factors of bone strength. The morphological and biochemical characteristics, along with the 
measured fracture resistance, are also correlated to age to determine which, if any, characteristics change throughout life. Differences in bone based on gender will also be analyzed, since previous studies have found that bone differs biochemically between male and female. 


\section{Materials and Methods:}

Materials:

HPLC grade water, acetonitrile, ethanol, methanol, acetic acid, and TEA were obtained from Fisher Scientific (Pittsburgh, PA, USA). Protein sequencing grade PITC was purchased from Sigma (St. Louis, MO, USA). One ml ampules of constant boiling hydrochloric acid and amino acid standard solutions were from Pierce (Rockford, IL, USA). The crystallized form of trans-4-hydroxyproline standard was from Sigma. Bovine serum albumin was obtained from Sigma. Airgas (Charleston, WV, USA) supplied the nitrogen and helium gases. $1 \mathrm{X}$ phosphate buffered saline $(8 \mathrm{~g} \mathrm{NaCl}, 0.2 \mathrm{~g}$ $\mathrm{KCl}, 1.44 \mathrm{~g} \mathrm{Na}_{2} \mathrm{HPO}_{4}$, and $0.24 \mathrm{~g} \mathrm{KH}_{2} \mathrm{PO}_{4}$ in $1.0 \mathrm{~L} \mathrm{H}_{2} \mathrm{O}$, pH 7.4) was made as needed and stored at $4^{\circ} \mathrm{C}$. Metrasystems provided the Pyrilinks ${ }^{\mathrm{TM}}$ enzyme-linked immunosorbent assay kits (Mountainview, CA, USA). 


\section{Bone Sample Selection:}

Cortical bone samples were removed from the tibia and the femur of cadavers donated by WVU Health Science / Ruby Memorial Hospital within three days post mortem. Few restrictions were placed on donors of bone samples. Donors were restricted to European descent. This was done in an attempt to better reflect the demographics of the state of West Virginia. African Americans represent only 3-5\% of the state's population while native Americans and Oriental Americans represent 1\% or less. Donors with long term disease, such as cancer or diabetes, were identified by death records and labeled to further examine sub-populations of the study, although none were identified in the present studies. Donor population included both sexes with ages ranging from 50 to 94 years old; median age of the sampled population was 79 , from a sample size of 13 .

Bone samples were taken from the medial and lateral cortices of the middiaphysis from both the tibia and the femur (Figure 11). Three pieces of bone were taken from each site, two for shear and tension fracture tests, and one from between them for analysis of its biochemical and morphological characteristics. Those samples used in biochemical analysis were cleansed of soft tissue and trabecular bone. The cortical bone pieces were then placed in acetone for 8-12 hours and shaken to extract any remaining lipids. The sample specimens were then freeze dried and milled to a fine powder under liquid nitrogen in a Spex percussion mill. The powdered bone samples were labeled according to age and sex of the donor, type of bone, location on the bone surface and from which leg. Samples were then stored at $-80^{\circ} \mathrm{C}$ until used. 
Figure 11

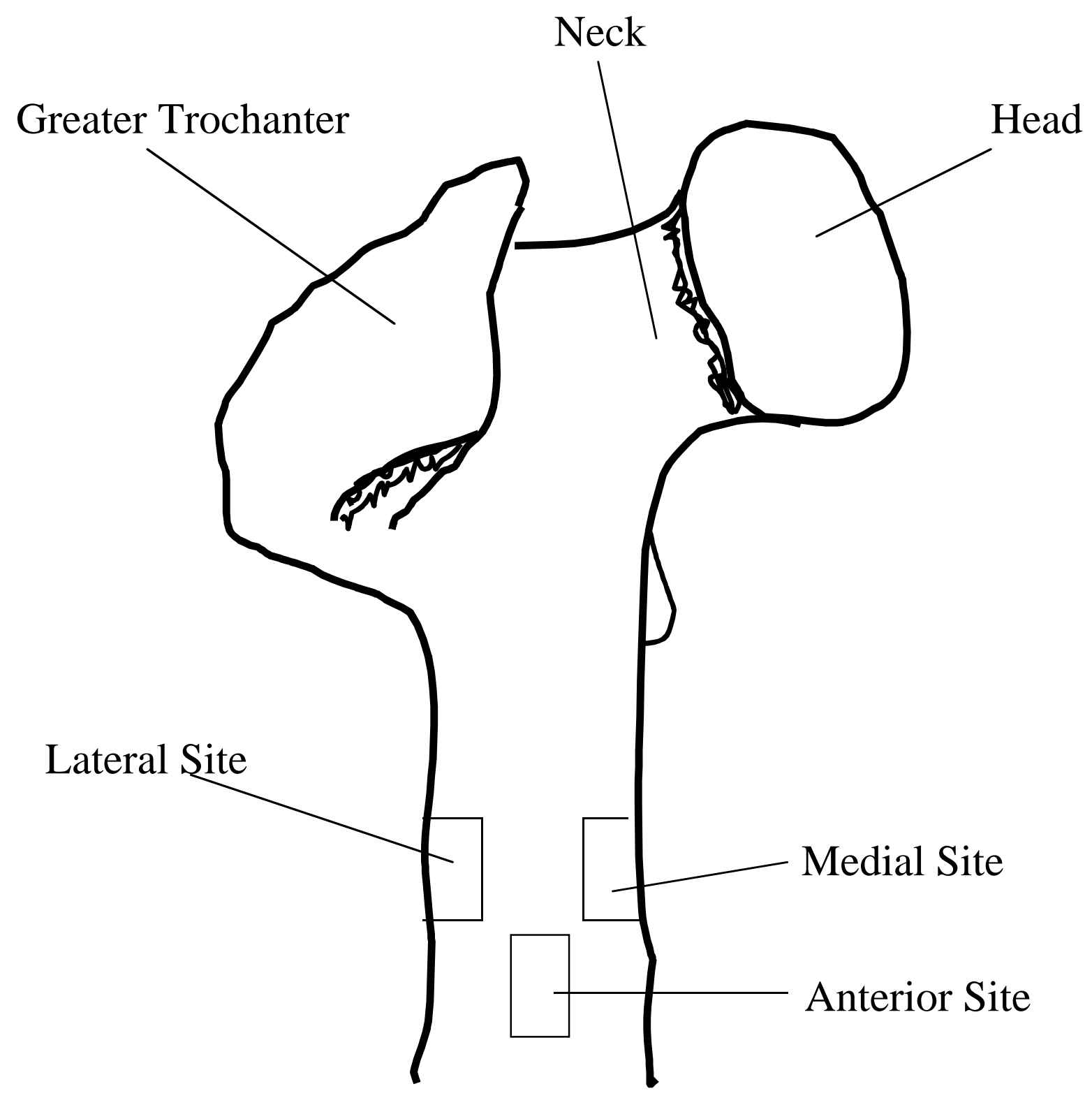

Figure 11. Cortical bone samples were taken from anterior, lateral, and medial sites of the mid-diaphysis from human femurs. 


\section{Bone Fracture:}

The inability of bone to effectively resist fracture is the essence of osteoporosis. In this study, two types of mechanically induced fractures were propagated on the bone slices, tension and shear (Figure 12). From these fractures, two indices of bone strength were obtained; specimen compliance, $(\mathrm{C})$ and the critical load, $(\mathrm{Pq})$. These indices were then used to calculate the critical strain energy release rate $\left(G_{c}\right)$, which correlates to bone's ability to resist fracture. The bone slices had a V-notch cut into them with a Vnotch saw blade; this is where the crack will develop. The slices were then saturated in $0.9 \% \mathrm{NaCl}$ while mounted on either a tension testing or shear-testing device. A linear variable displacement transducer and a load cell monitored the displacement and load, respectively. Crack development is measured by a traveling microscope attached to the load frame, and by a crack propagation strain gauge. Loading is performed at a rate of $0.002 \mathrm{~cm} / \mathrm{min}$. The results for specimen compliance and critical load were reported in the Ph.D. thesis by Yeni (72). The calculated critical strain energy release rates $\left(G_{c}\right)$ were then used in comparisons to results obtained from the present studies measuring type-I collagen amount and pyridinium crosslink content.

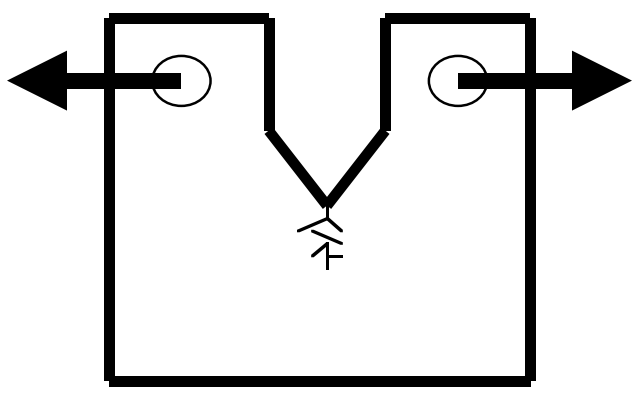

Tension

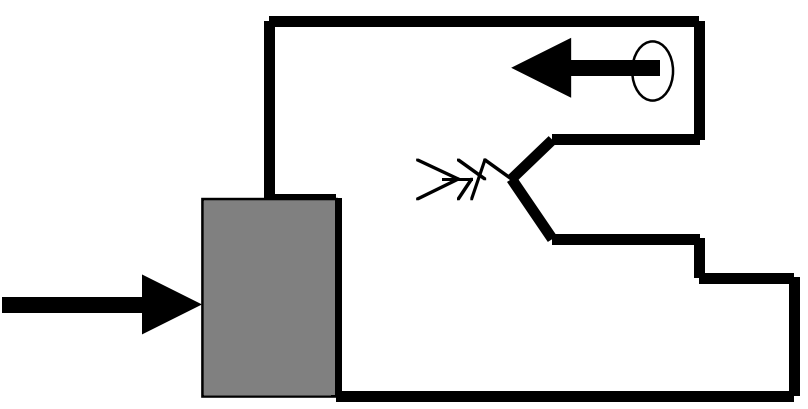

Shear 


\section{Hydrolysis of Bone:}

Samples were hydrolyzed by methods according to Valais et al. $(45,66)$. Ten mgs of powdered cortical bone was placed in a Pierce hydrolysis tube along with $1.0 \mathrm{ml}$ of $6 \mathrm{~N}$ hydrochloric acid. Oxygen gas was removed for a clean hydrolysis by alternating five rounds of vacuum pressure and nitrogen gas. Hydrolysis tubes were sealed under a reduced pressure of $\sim 600 \mathrm{~mm} \mathrm{Hg}$ with an Emerson Compressor / Vacuum pump. The samples were then heated to $108^{\circ} \mathrm{C}$ for 20 hours in a Pierce Reacti-therm heating module in the absence of light. After the samples were hydrolyzed, two $300 \mu$ l aliquots were taken and dried under a nitrogen stream. One sample was used in the quantification of pyridinium crosslinks in bone, and the other in the determination of the fractional composition of type-I collagen per bone sample. 


\section{Derivitization and Quantification of Type-I Collagen:}

High performance liquid chromatography (HPLC) is a technique used to separate molecules by sending them, in solution, through a column packed with particles at a high pressure. These experiments utilized reverse phase HPLC using octadecylsilane (ODS) beads to separate phenylthiocarbamyl-labeled amino acids from hydrolyzed cortical bone. The ODS beads packed throughout the column will separate the derivitized amino acids based on size and shape as specified by the "R" group. The solvent system used was composed of two solutions: the aqueous mobile phase, and a washing buffer. Derivitized bone hydrolysates were redissolved and diluted in the mobile phase and injected through a port into the HPLC. The ODS beads separated amino acids within the sample as the mobile phase carried them across the column at a high pressure. After separation, a detector using UV light absorbance records the PITC-derivitized amino acids by elution time, and this is matched to the known elution times of specific amino acids. The amount of the light absorbed and the period of time that the light is absorbed is proportional to the amount of amino acid present, and this is monitored by the chart recorder. Following this, the solvent is switched to the washing buffer to elute any residual sample left on the column, thereby regenerating the column for additional usage. In these studies, the column was typically replaced following two hundred sample injections. A standard curve was used to determine an amino acid's amount in an unknown sample. Hydroxyproline, the amino acid used to determine collagen content, elutes at $\sim 4.5$ minutes as determined by injecting hydroxyproline alone, and also when mixed with bovine serum albumin (BSA) hydrolysates. The addition of hydroxyproline to BSA, a 
protein that does not contain hydroxyproline, tested whether hydroxyproline's elution time was changed in the presence of other PITC-labeled amino acids in order to confirm that hydroxyproline could be accurately detected.

The derivitization of the free amino acids from the acid hydrolyzed bone was performed according to the methods described by Bidlingmeyer et al. (74). The nitrogen dried residue of the $300 \mu \mathrm{l}$ aliquot of hydrolyzed bone was dissolved in $10 \mathrm{mls}$ of waterethanol-triethylamine (TEA) (2:2:1 ratio), and filtered through a Corning 0.20 micron cellulose acetate membrane to remove any large mineral crystals that might have impeded the flow through the column. Five $50 \mu \mathrm{l}$ samples of the redissolved specimens were taken and dried in a Savant ISS 100 Speed-Vac Concentrator. Each sample was then redissolved with $50 \mu 1$ of PITC-solution (ethanol-water-TEA-phenolisothiocyanate (PITC) (7:1:1:1 ratio). The amino acids in this solution react to produce phenylthiocarbamyl derivatives of each amino acid (Figure 13). PITC was used as the derivitizing agent for its efficiency in enzyme kinetics, derivative stability, and sensitivity in the detection of amino acids from protein hydrolysates at the pmol level (74). The derivitization reaction of the samples was run for 30 minutes at room temperature, and then dried again using the Savant Concentrator and stored at $4^{\circ} \mathrm{C}$ until analysis.

For quantification, the PITC derivitized amino acids were resuspended in the mobile phase of the HPLC (140 mM sodium acetate solution-water-acetonitrile [90:4:6] with $0.5 \mathrm{ml}$ TEA added per $1.0 \mathrm{~L}$ of solution, then titrated to a $\mathrm{pH}$ of 6.35 ). Glacial acetic acid was used to adjust the $\mathrm{pH}$ of the mobile phase. The mobile phase was degassed for 30 minutes under reduced pressure $(\approx 600 \mathrm{~mm} \mathrm{Hg})$, and then constantly helium sparged to remove any remaining larger air bubbles that could "crack" the column or impede the 
flow of the mobile phase. A flow rate of $0.8 \mathrm{ml} /$ minute was used to separate the specific PTC-amino acids with a pressure across the column between 2000-2500 PSI. After the sample had passed the detector, the column was cleansed with an acetonitrile-water solution [60:40] for twenty minutes. Following this, the column was equilibrated by the mobile phase for twenty minutes prior to the next injection. The HPLC used was a Waters 600 E System Controller with a Nova-pak column (300 mm by $3.9 \mathrm{~mm}$ ) with a pore size of $60 \AA$ and a particle size of $4 \mu \mathrm{m}$. The PTC-amino acids were detected with a Waters 486 Tunable Absorbance Detector using a deuterium lamp set at a wavelength of $254 \mathrm{~nm}$ and AUFS set at 0.1. A Waters 746 Data Module recorded the deflection of absorbance and the elution times of samples.

\section{Phenolisothiocyanate}

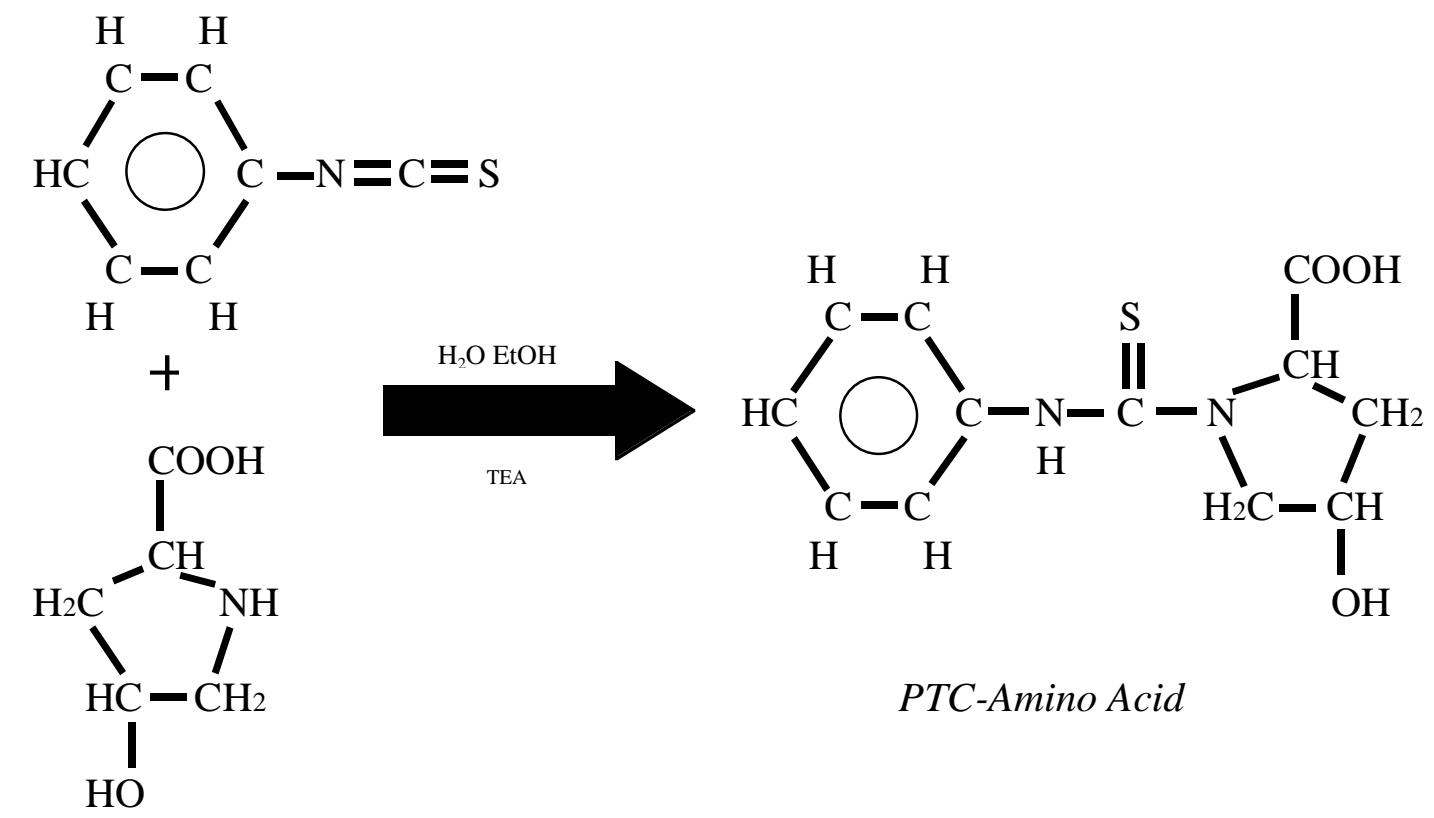

\section{4-Hydroxyproline}

Formation of phenylthiocarbamyl hydroxyproline 


\section{Standards:}

Known amounts of PITC derivitized hydroxyproline were separated by HPLC to establish a concentration curve for each HPLC based determination of hydrolyzed bone hydroxyproline content. Amounts of PTC-Hyp as low as 3.0 nanograms were reproducibly detected, and a linear absorbance range between 10 nanograms and 50 nanograms was used to produce the standard curves. The correlation coefficient $\left(\mathrm{r}^{2}\right)$ of the standard curves was typically 0.95 or better. The determination of hydroxyproline had assay variabilities between $0.0 \%$ and $12.7 \%$ for intraassay $(\mathrm{N}=19$; mean of $3.89 \%$; median $2.40 \%)$ and between $1.6 \%$ and $20.8 \%$ for interassay $(\mathrm{N}=10$; mean of $8.88 \%$; median of $7.55 \%)$.

Hydroxyproline was added to a bovine serum albumin (BSA) solution. BSA is a protein that does not contain hydroxyproline and the sampled was hydrolyzed and derivitized as described. This control experiment was performed to determine if other amino acids affected the detectability, reproducibility, or the retention time of PTChydroxyproline. It's detectability and the reproducibility of the assays were unchanged. The PTC-hydroxyproline was recorded at similar retention times irrespective of the BSA, however slight variations of the elution times of all amino acids did occur. These variations appear to have been the product of column pressure that fluctuated slightly from day to day for unknown reasons rather than effects from the presence of other amino acids. Bone samples were diluted prior to injection to an estimated 30 nanograms of hydroxyproline per $10.0 \mu \mathrm{l}$ injection. Appropriate dilutions were estimated from the reports that bone is $35 \%$ organic matrix, $90 \%$ of which is type-I collagen, and assuming 
that the proportionality constant of hydroxyproline is $0.14 \mu \mathrm{gs}$ for $1.0 \mu \mathrm{g}$ of type-I collagen (45). 


\section{Calculations:}

Hydroxyproline

The amount of hydroxyproline per ten milligrams of bone was determined by the formula:

\section{$\left(\mathrm{N} / 1 \times 10^{-5}\right) \times 14.667=\mathrm{H}$}

Where " $\mathrm{N}$ " is the number of nanograms of hydroxyproline in a $10 \mu$ injection as determined by a standard curve.

" $1 \times 10^{-5}$ " is the amount injected (in liters) into the HPLC.

"14.667" is the dilution factor.

" $\mathrm{H}$ " is the amount of hydroxyproline in 10.0 milligrams of cortical bone.

\section{Type-I Collagen}

The amount of type-I collagen per ten milligrams of bone was then determined by the formula:

\section{$\mathrm{H} / \mathbf{0 . 1 4}=\mathrm{C}$}

Where "H" is the amount of hydroxyproline in 10.0 milligrams of bone.

" 0.14 " is the proportionality constant of hydroxyproline to type-I collagen.

"C" is the amount of type-I collagen in 10.0 milligrams of cortical bone. 


\section{$\underline{\text { Pyridinium }}$}

Pyridinium content in the bone samples was measured by EIA as described in a following section. The amount of pyridinium per 10 milligrams of cortical bone was determined by the formula:

$$
X * 6.67=P
$$

Where " $\mathrm{X}$ " is the amount of pyridinium in nanomoles as determined by the standard curve.

"6.67" is the dilution factor for the EIA.

"P" is the nanomoles of pyridinium per 10 milligrams of cortical bone.

\section{$\underline{\text { Ratio of Pyridinium to Type-I Collagen }}$}

The ratio of pyridinium crosslinks to collagen content is determined by the equation:

\section{C / 300,000 = moles of Type-I Collagen.}

Where "C" is the amount of collagen in milligrams.

"300,000" is the molecular weight of type-I collagen.

\section{moles of Pyridinium / moles of Type-I collagen = Ratio.}

This ratio has been estimated to be about 0.3 moles pyridinium per 1.0 mole collagen in calcified bone $(26,57,63,75)$. 


\section{Critical Strain Energy Release Rate}

Fracture resistance for the samples used in the present studies were measured as described by Yeni (72). Bone's ability to resist fracture is measured as the critical strain energy release rate for both tension $\left(\mathrm{G}_{\mathrm{Ic}}\right)$ and shear $\left(\mathrm{G}_{\mathrm{IIC}}\right)$. This is determined by measuring the compliance $(\mathrm{Cm})$ as the inverse of the slope of the unloading-reloading load (P) and the deflection (d) expressed by the formula:

\section{$\mathbf{C m}=\mathrm{d} / \mathrm{P}$}

The compliance measurements are located at machined designated rate locations 0.254 $\mathrm{mm}$ apart from each other. Linear regression analysis was used to calculate compliance from the unloading-reloading curves by determining the intersection with a line whose slope is 0.95 . The compliance is then used to define the critical strain energy release rate by the formula used in LEFM:

$$
G_{\text {Ic }}=\frac{P_{Q}{ }^{2}}{2 B} \frac{d C m}{d a}
$$

Where "B" is the specimen thickness, " $\mathrm{P}_{\mathrm{Q}}{ }^{2 "}$ is the critical load corresponding to crack growth, and "dCm / da" is the slope of the curve for compliance vs. crack growth at specific crack positions. For the femur and tibia, "a" is $8.0 \mathrm{~mm}(72) . \mathrm{G}_{\mathrm{Ic}}$ and $\mathrm{G}_{\mathrm{IIC}}$ are used to describe resistance to tension and shear fracture, respectively, and were related to collagen and pyridinium amounts found in cortical bone in the present studies. 


\section{Quantification of Pyridinoline and Deoxypyridinoline Crosslinks:}

A dried $300 \mu \mathrm{l}$ sample of hydrolyzed bone was redissolved in $1.0 \mathrm{ml}$ of filtered Phosphate Buffered Saline (PBS) shielded from light with an aluminum foil wrap, and stored at $-80^{\circ} \mathrm{C}$ until used. Free pyridinoline and deoxypyridinoline crosslinks were measured using the Metra Biosystems Pyrilinks ${ }^{\mathrm{TM}}$ assay that has a detection limit of 1.1 nM. Although the Pyrilinks assay is designed to be used clinically to detect pyridinium in urine, no problems were anticipated in the assay's ability to detect free crosslinks from hydrolyzed bone, since approximately $70 \%$ of pyridinium in urine is peptide bound and an acid hydrolysis step is needed to generate free crosslinks. The acid hydrolysis step for urine uses $6 \mathrm{~N}$ hydrochloric acid and similar times and temperatures as were used in the hydrolysis of bone. In the hydrolysis of urine, destruction levels of crosslinks have been found to vary between $20-60 \%$ (75). It is unknown if any degradation of pyridinium crosslinks occurs by the direct hydrolysis of powdered bone. Both crosslinks have a combined estimated concentration of 0.3 moles / 1.0 mole of type-I collagen $(26,57,63)$. The Pyrilinks kit is a competitive inhibition assay that uses alkaline phosphotase conjugated pyridinoline crosslinks with P-nitrophenol-phosphate (PNPP) as the substrate. Pyridinium crosslinks from the bone samples irreversibly bind to the antibodies on the plate and remain after washing. An excess of alkaline phosphotase conjugated crosslinks are then added and binds to all unbound antibodies. After washing off the unbound enzyme conjugated crosslinks, PNPP is added to the wells and the alkaline phosphotase activity produces a color change that is measured using the BioRad model 450 microplate reader. Absorbance was measured at a wavelength of $405 \mathrm{~nm}$. Each sample's absorbance 
was compared with a standard curve $\left(r^{2} \geq 0.97\right)$ produced for each assay. From these measurements, the pyridinium content for 10.0 milligrams of cortical bone was calculated. 


\section{Statistical Analysis:}

Statistical analysis of standard curves and predicted values for type-I collagen and pyridinium crosslinks were determined using a simple linear regression analysis performed by JMP software (SAS Institute, Cary, N.C.). Standard curves in the HPLC hydroxyproline assays had a $\mathrm{r}^{2}$ value of 0.95 or greater. Analysis of collagen concentration and pyridinium content, and their relation to fracture resistance, was done using a linear and multiple regression analysis by JMP software and Excel software. Significance was set at Prob. $>\mathrm{F}=0.05$. Linear regression was used to determine compliance and LEFM for fracture resistance (72). Intra-assay and inter-assay variabilities for collagen and pyridinium amounts were determined with each sample's predicted value, along with standard errors. 


\section{Results:}

Type-I collagen's role in bone formation is well documented, although its relationship to bone strength has not yet been fully determined. Pyridinium crosslinks in collagen have been found to be excellent markers for bone turnover, but their effect on bone strength is unclear. In this study, cortical bone from the human femur was tested for the amount of type-I collagen present in 10.0 milligrams of bone, and the pyridinium crosslink content of that collagen. Type-I collagen and pyridinium amounts were compared against bone's resistance to both tension and shear fracture (measured previously by Yeni (72)), to better understand their roles in bone strength. Type-I collagen and pyridinium amounts were also analyzed against the age and sex of the bone donor to determine if any age-related or sex-related differences could be determined; however, the number of samples per decade varied widely and results should be cautiously interpreted. Although size, shape, and mass of cortical bone varies by position around the femur, cortical bone samples from the anterior, lateral and medial sites on the femur were pooled for collagen amounts. Lateral and medial sites were pooled for pyridinium amounts, and for tension and shear fracture resistance (9). This group-wise, location pooled data comparisons were done because the fractional composition of collagen and pyridinium content in these powdered cortical bone samples were the same $(\mathrm{p}=0.26 ; \mathrm{N}=13, \mathrm{p}=$ $0.16 ; \mathrm{N}=23$; Graph 1; collagen and pyridinium respectively). Data were also analyzed by sex to determine if the sex of the donor related to the force needed to fracture.

A significant relationship between type-I collagen content and the force needed for tension fracture was demonstrated $(\mathrm{p}=0.046 ; \mathrm{N}=8$; Graph 2). No significant 
relationship between type-I collagen content and shear fracture resistance was identified $(\mathrm{p}=0.13 ; \mathrm{N}=8 ;$ Graph 3).

Type-I collagen content was not significantly related to the donors age $(p=0.12$; $\mathrm{N}=13$; Graph 4), although additional data may alter this conclusion. A previous study showed that age did significantly correlate with the force needed for both tension and shear fractures $(\mathrm{p}=0.04 ; \mathrm{N}=28$; and $\mathrm{p}=0.006 ; \mathrm{N}=31$, respectively) (72). A model comparing the interaction of the percentage of type-I collagen and the age of donor failed to show any effect on the force needed for either tension or shear fracture. The relationship of type-I collagen and age combined, and their effect on tension fracture resistance and shear fracture resistance were not significant $(\mathrm{p}=0.15 ; \mathrm{N}=8 ; \mathrm{p}=0.71 ; \mathrm{N}$ $=8$ respectively).

Male donor derived bone exhibited a higher amount of type-I collagen per ten mg cortical bone $(2.61 \pm 0.08 \mathrm{mg}, \mathrm{N}=7)$ than did female derived samples $(2.34 \pm 0.08 \mathrm{mg}$, $\mathrm{N}=6)(\mathrm{p}=0.038 ; \mathrm{N}=13$; Graph 5). When the combined effect of type-I collagen amount and the sex of the donor was tested, no influence on fracture resistance was found. P values for these combined effects (percent collagen and sex of donor) on tension fracture and on shear fracture were $0.40(\mathrm{~N}=8)$, and $0.25(\mathrm{~N}=8)$, respectively. The amount of pyridinium could not predict the force needed for tension fracture in pooled samples $(\mathrm{p}=0.32 ; \mathrm{N}=19 ; \mathrm{Graph} 6)$. However, when sub-dividing the samples based on sex, pyridinium concentration was predictive for tension fracture resistance in males $(\mathrm{p}=0.048 ; \mathrm{N}=10)$, but not in females $(\mathrm{p}=0.91 ; \mathrm{N}=9$; Graph 7-A, 7-B). Interestingly, the combined effects of pyridinium concentration and sex had no significant effect on bone's ability to resist tension fracture $(\mathrm{p}=0.24 ; \mathrm{N}=19)$. Bone-site 
related pyridinium concentrations also showed no relationship to tension fracture resistance (medial site: $\mathrm{p}=0.16 ; \mathrm{N}=8$ ); lateral site: $\mathrm{p}=0.85 ; \mathrm{N}=11$ ).

Relating pyridinium concentration to shear fracture gave similar results to those obtained for tension fracture. No correlation existed between pyridinium concentration and force needed for shear fracture in the pooled samples $(\mathrm{p}=0.15 ; \mathrm{N}=20$; Graph 8). However, in male bone, pyridinium concentration could predict shear fracture resistance $(\mathrm{p}=0.0287 ; \mathrm{N}=10)$, but not in females $(\mathrm{p}=0.71 ; \mathrm{N}=10 ;$ Graph 9-A, 9-B). No combinatorial effects of sex and pyridinium concentration on shear fracture were found $(\mathrm{p}=0.60 ; \mathrm{N}=20)$. Bone-site pyridinium concentration could not predict shear fracture force. Medial site samples gave a $\mathrm{p}=0.136(\mathrm{~N}=9)$, while the lateral site gave a $\mathrm{p}=0.26$ $(\mathrm{N}=11)$.

Pyridinium concentration did not relate to the sex of the donor $(\mathrm{p}=0.24 ; \mathrm{N}=23)$. The measured value of pyridinium in female samples was 939 nanomoles $\pm 72.5 \mathrm{~nm} /$ 10.0 milligrams of bone $(\mathrm{N}=10)$ and in males was 823 nanomoles $\pm 63.5 \mathrm{~nm} / 10.0$ milligrams of bone $(\mathrm{N}=13)($ Graph 10$)$.

Medial and lateral bone sample pyridinium concentrations were compared. Pyridinium content in the lateral sample was $945 \pm 68$ nanomoles and in the medial site was $808 \pm 65$ nanomoles $(\mathrm{p}=0.16 ; \mathrm{N}=23$; Graph 11$)$. When comparing whether the site from which the bone slice was removed effected fracture resistance, the results showed that the force needed for tension fracture was equal between the bone from the lateral or medial site, (Graph 12-A). Force needed for shear fracture, however, was measured for the lateral site $\left(\mathrm{Gii}_{\mathrm{c}}=1657 \pm 242 ; \mathrm{N}=15\right)$, and for the medial site $\left(\mathrm{Gii}_{\mathrm{c}}=\right.$ 
$2296 \pm 234 ; \mathrm{N}=16$ ). These data on site-specific force needed for shear fracture only approached significance $(\mathrm{p}=0.07 ; \mathrm{N}=31$; Graph 12-B).

Force needed to fracture was donor-sex related for tension fractures $(\mathrm{p}=0.04 ; \mathrm{N}$ $=28)$, but not for shear fracture $(\mathrm{p}=0.12 ; \mathrm{N}=31$; Graph 13-A, 13-B). The male specimens were more resistant to tension fracture forces (72).

No correlation was evident between donor age and bone pyridinium concentration for all samples $(\mathrm{p}=0.63 ; \mathrm{N}=23$; Graph 14$)$. No correlation was seen in the male samples $(\mathrm{p}=0.95 ; \mathrm{N}=13$; Graph $15-\mathrm{A})$; the data for female bone suggested that pyridinium concentration may decrease with donor age, although a significant relationship could not be demonstrated $(\mathrm{p}=0.11 ; \mathrm{N}=10$; Graph 15-B). Sample site analysis also revealed no effect of age to pyridinium concentration.

Collagen and pyridinium content were compared against percent mineral and crystallinity, two components of the inorganic fraction of bone. Collagen content was significantly correlated to crystallinity (an indice of crystal size and perfection) $(\mathrm{p}=0.04$; $\mathrm{N}=7$; Graph 16). Collagen content did not relate to percent mineral content $(\mathrm{p}=0.135$; $\mathrm{N}=7$; Graph 17). Pyridinium did not relate to crystallinity $(\mathrm{p}=0.92 ; \mathrm{N}=9)$ nor to percent mineral $(\mathrm{p}=0.42 ; \mathrm{N}=9)(\mathrm{Graph} 18-\mathrm{A}, 18-\mathrm{B})$. For both tension and shear types of fracture, the ratio of pyridinium to collagen showed no ability to predict bone strength for tension fracture $(\mathrm{p}=0.96 ; \mathrm{N}=8)$; nor for shear fracture $(\mathrm{p}=0.27 ; \mathrm{N}=8)($ Graph 19A, 19-B). However, when the combined effect of collagen and pyridinium were compared against fracture resistance, an indication of a relationship appeared for tension fracture ( $\mathrm{p}=0.066 ; \mathrm{N}=8$; Graph 20). This relationship was not seen in shear fracture ( $\mathrm{p}$ $=0.126 ; \mathrm{N}=8$ ). Comparing the pyridinium:collagen ratio to the sex of the donors also 
provided an indication of a potentially significant correlation $(\mathrm{p}=0.06 ; \mathrm{N}=10)$ with the ratio in females equal to $0.117 \pm 0.012$, and in males $0.083 \pm 0.01$ (Graph 21). The age of the donor had no effect on the ratio of pyridinium to collagen $(p=0.20 ; N=10 ;$ Graph 22). 


\section{Collagen Fractional Amount by Location on Femur}

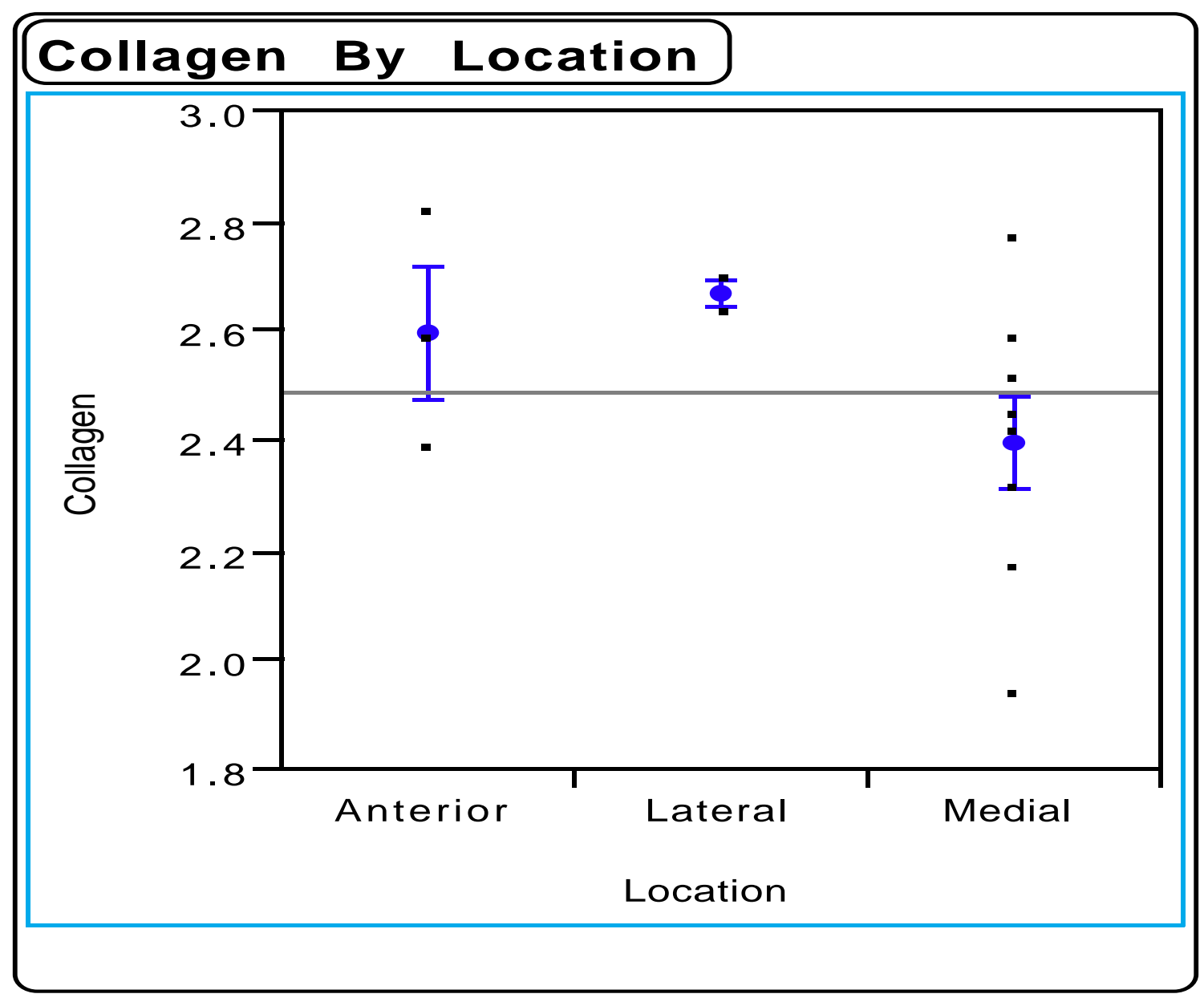

Graph 1. Collagen fractions (mgs/ $10.0 \mathrm{mgs}$ cortical bone) did not vary significantly by site from which the bone was removed $(p=0.26$; $\mathrm{N}=13$ ). 


\section{Type-I Collagen Content by Tension Fracture}

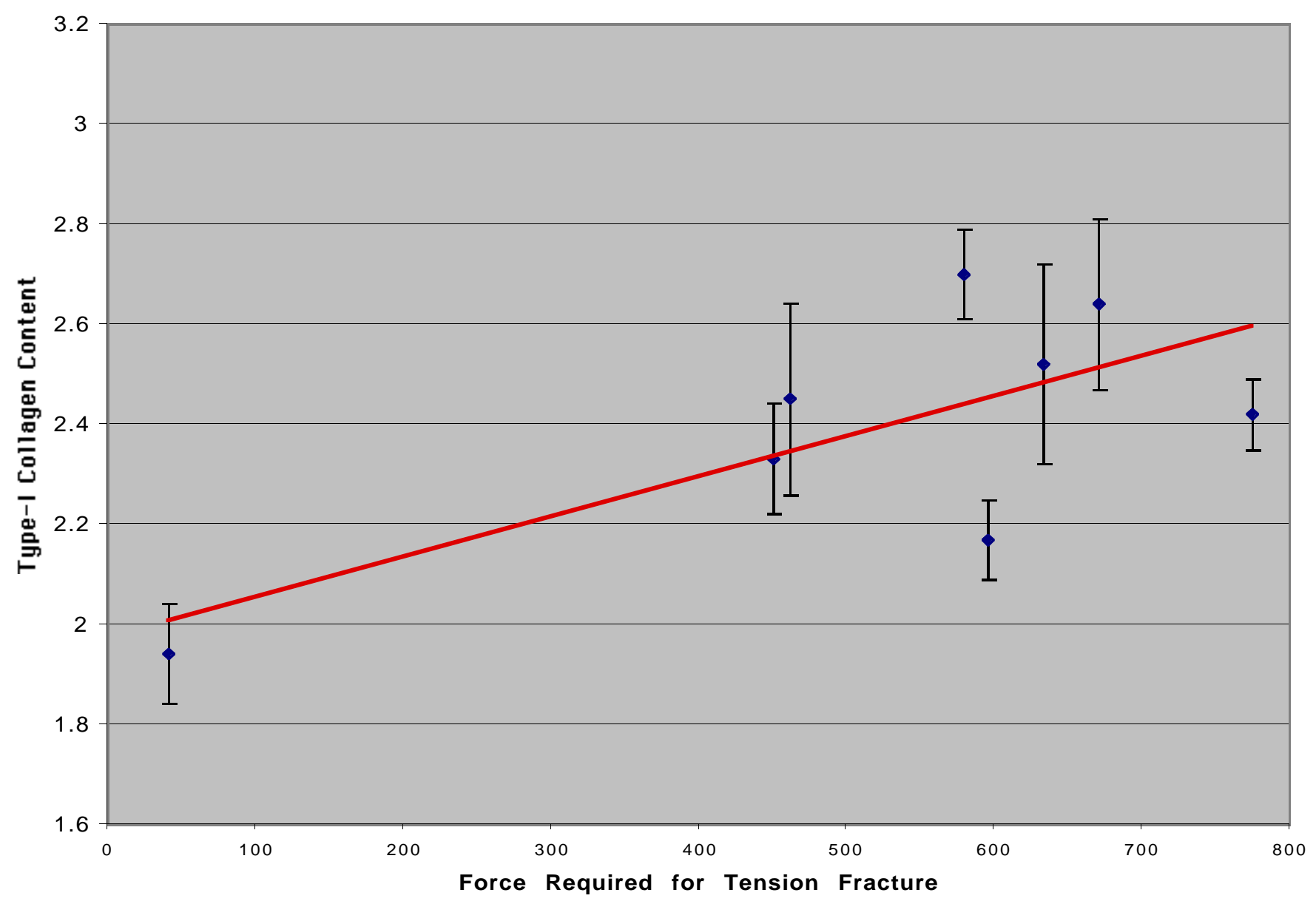

Graph 2. Type-I collagen (mgs/ $10.0 \mathrm{mgs}$ bone) and its effect on force required for tension fracture. The data were statistically significant $(\mathrm{p}=$ $0.046 ; \mathrm{N}=8$ ). 


\section{Type-I Collagen Content by Shear Fracture}

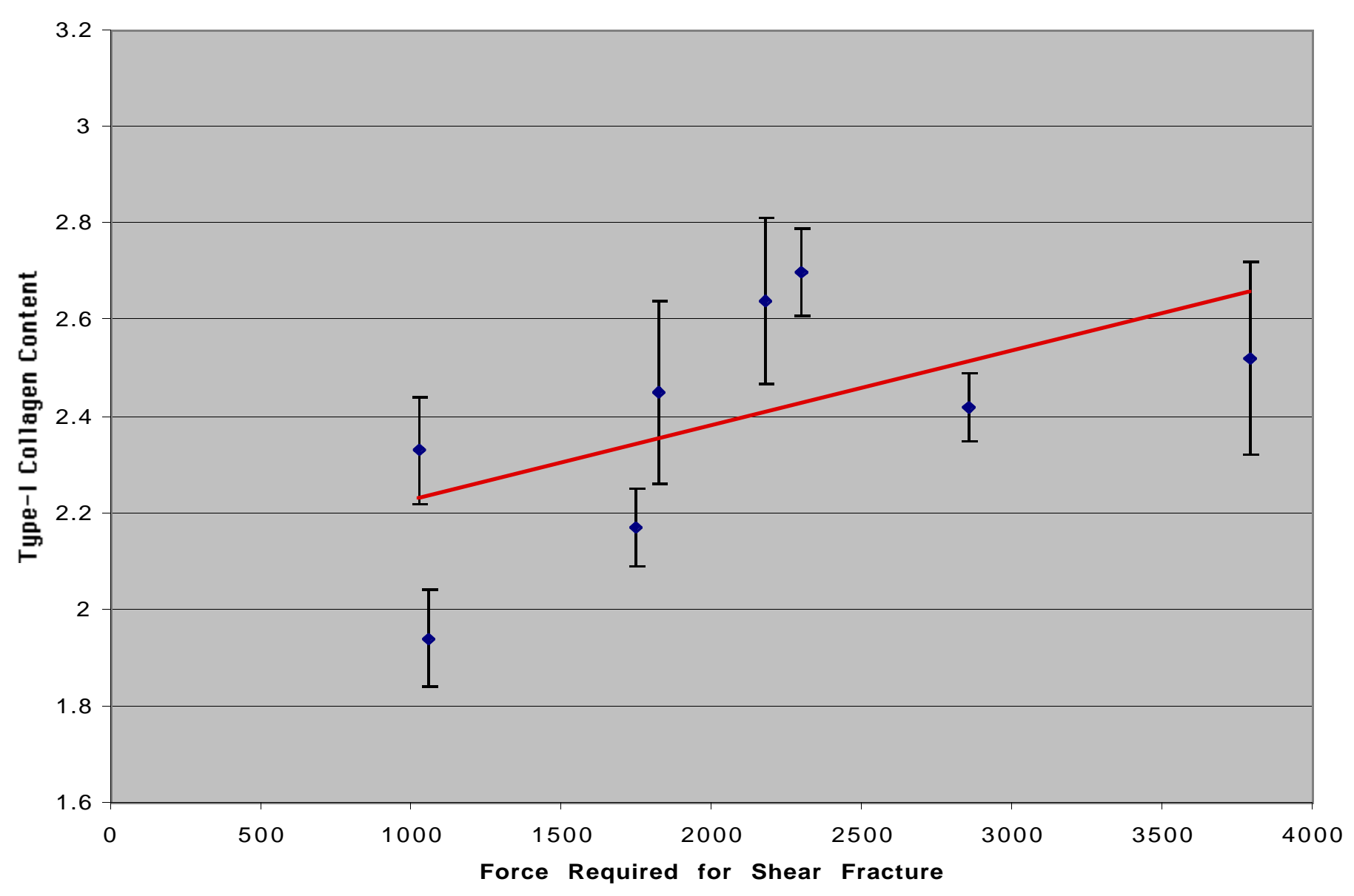

Graph 3. Type-I collagen (mgs/ $10.0 \mathrm{mgs}$ bone) and its effect on force required for shear fracture. The relationship was not significant $(\mathrm{p}=0.138$; $\mathrm{N}=8$ ). 


\section{Type-I Collagen Content by Age}

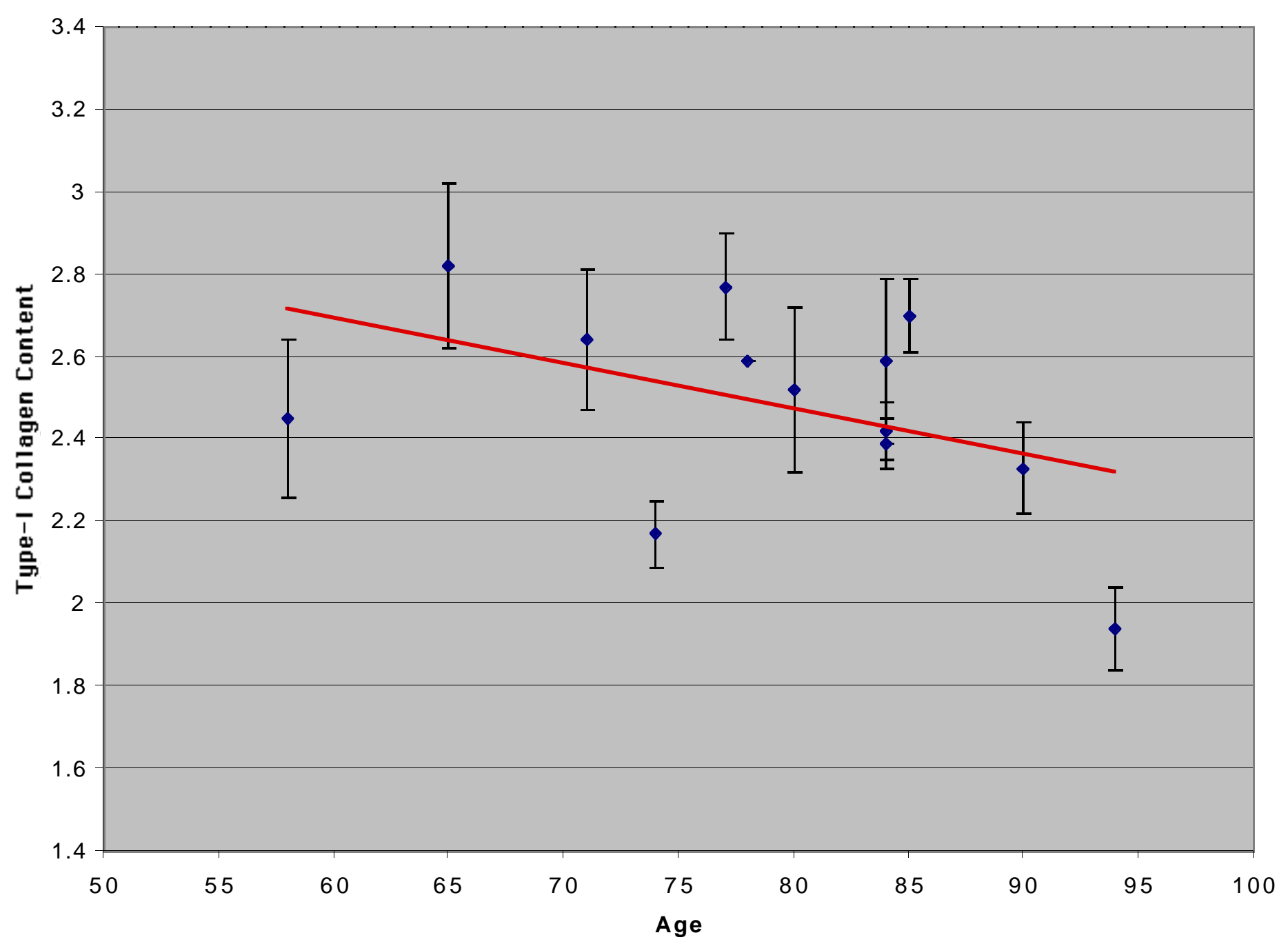

Graph 4. With increasing age, the fractional amount of type-I collagen in the femoral cortical bone appears to decrease. However, the data only approached significance $(\mathrm{p}=0.12 ; \mathrm{N}=13)$. 


\section{Type-I Collagen Content by Sex}

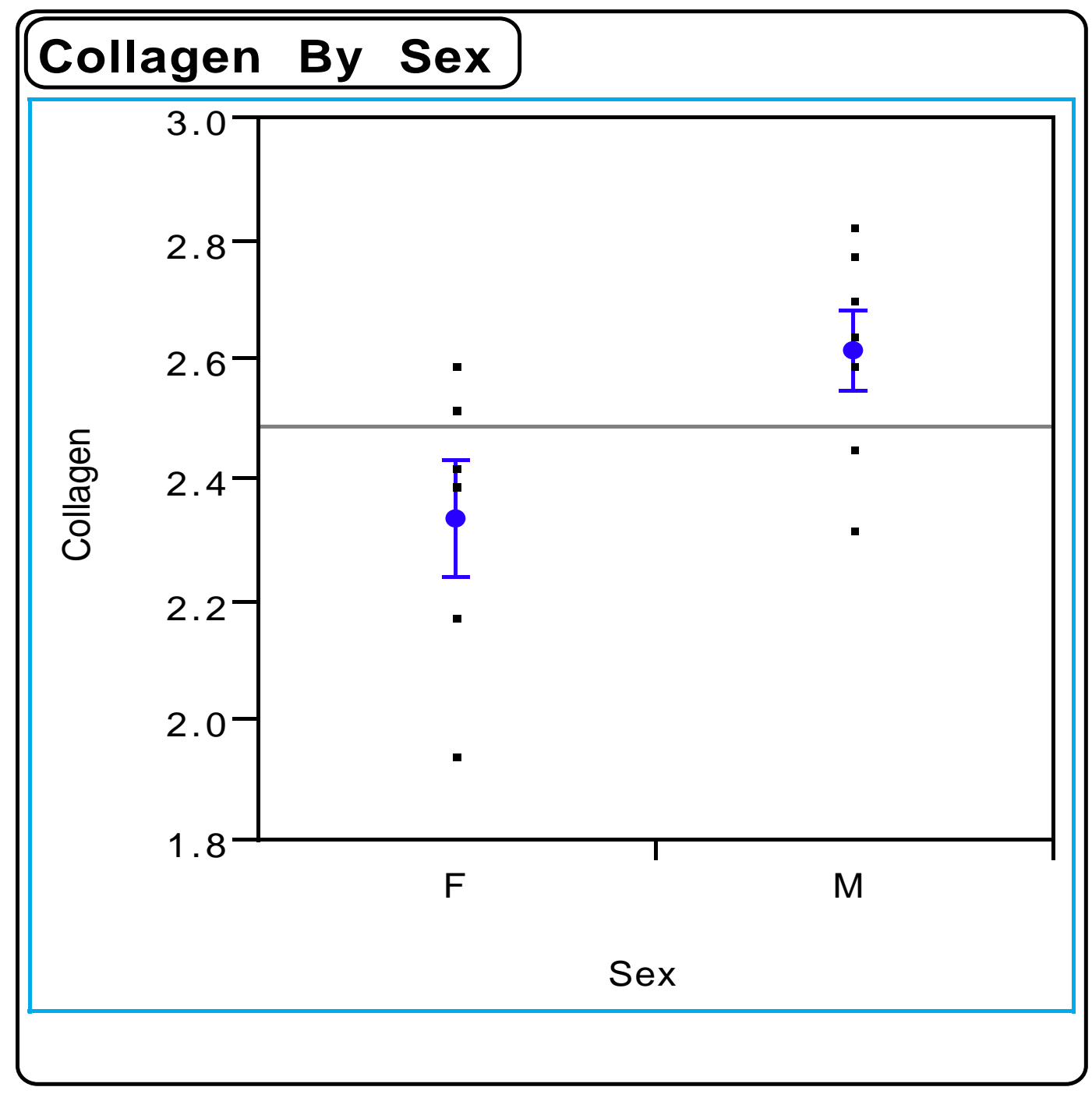

Graph 5. Type-I collagen content (mgs/ $10.0 \mathrm{mgs}$ of cortical bone) from the femur was significantly related to the sex of the donor $(\mathrm{p}=0.038 ; \mathrm{N}=13)$. 


\section{Pyridinium Content by Tension Fracture}

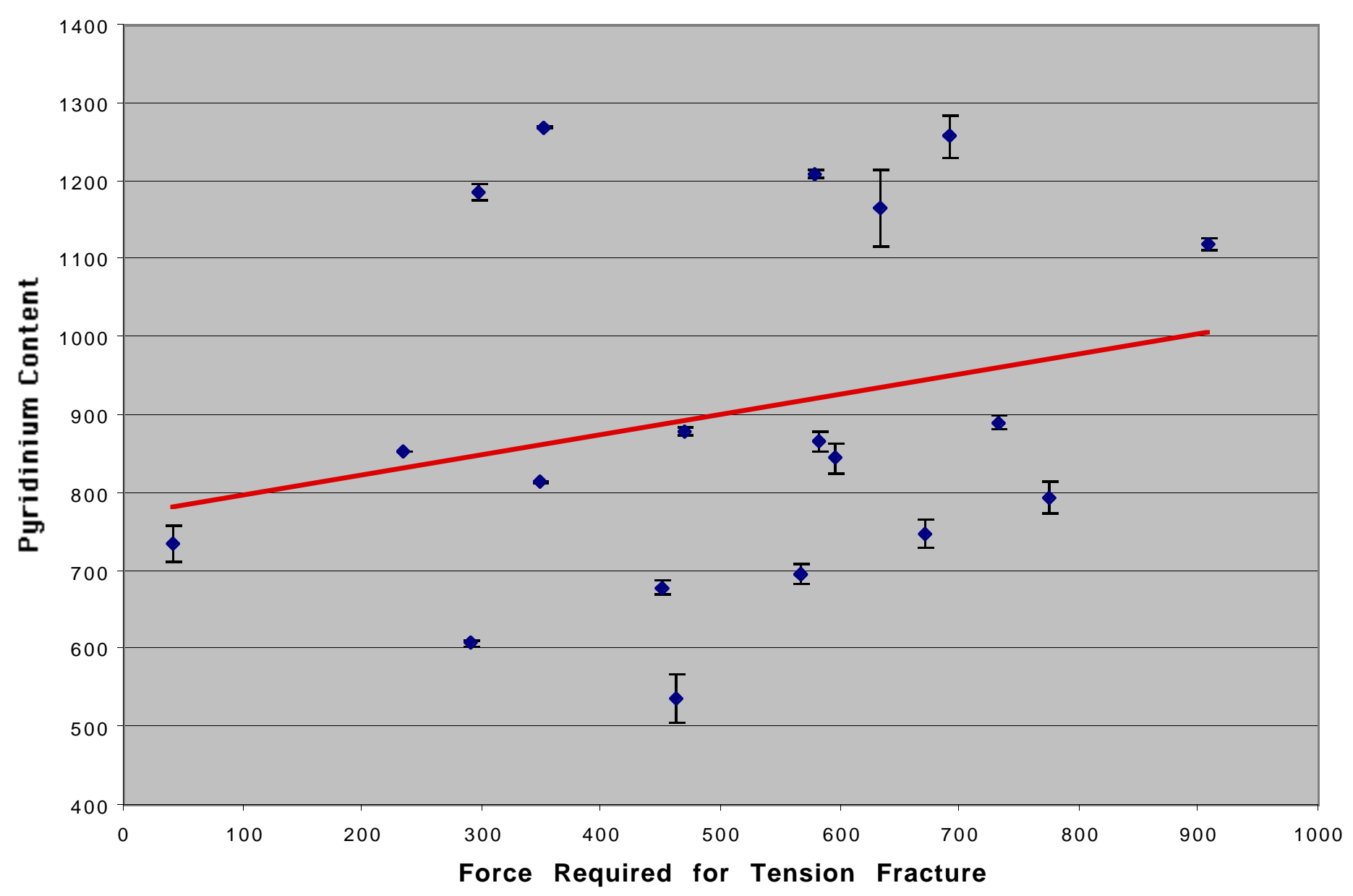

Graph 6. Pyridinium content ( $\mathrm{nmol} / 10.0 \mathrm{mgs}$ bone) showed no correlation to the force required for tension fracture in cortical bone of the femur shaft. Data represents all donors sampled $(\mathrm{p}=0.32 ; \mathrm{N}=19)$. 


\section{Pyridinium Content verse Tension Fracture by Gender}

\section{Graph 7-A}

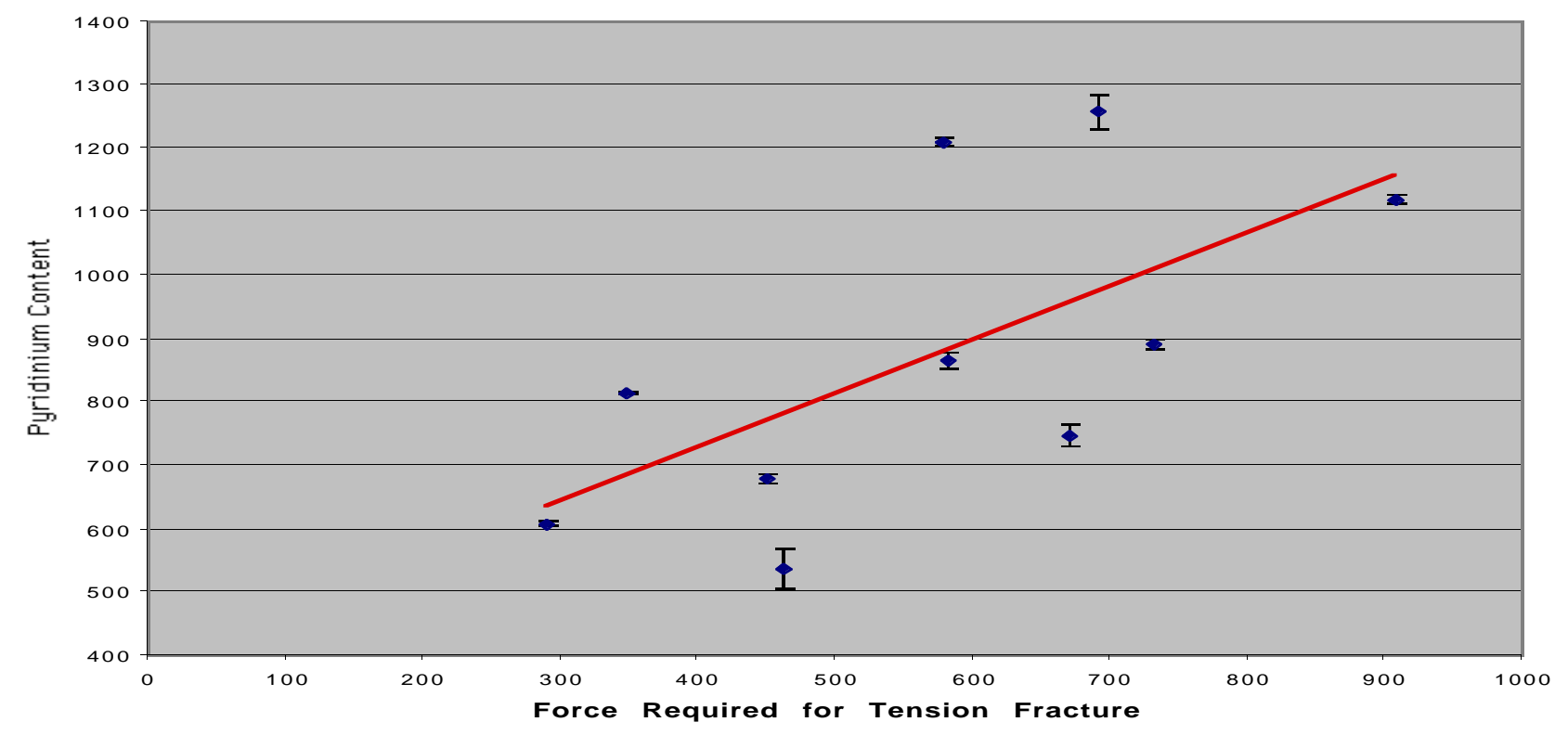

\section{Graph 7-B}

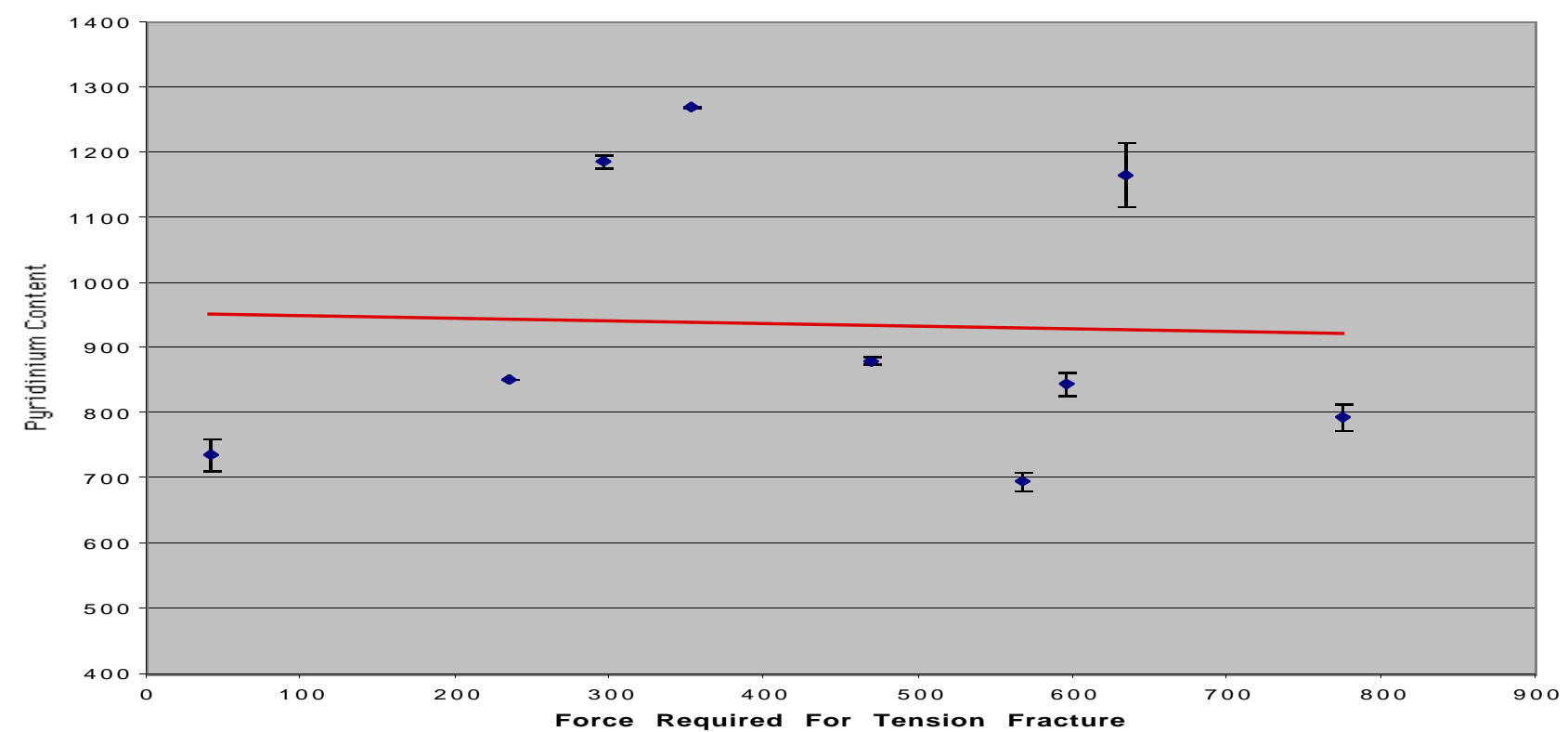

Graph 7-A. Pyridinium content (nmol/ $10.0 \mathrm{mgs}$ bone) predicted tension fracture in males donors ( $\mathrm{p}=0.048 ; \mathrm{N}=10)$, but not in females donors (Graph 7-B) $(\mathrm{p}=.91 ; \mathrm{N}=13)$. 


\section{Pyridinium Content by Shear Fracture}

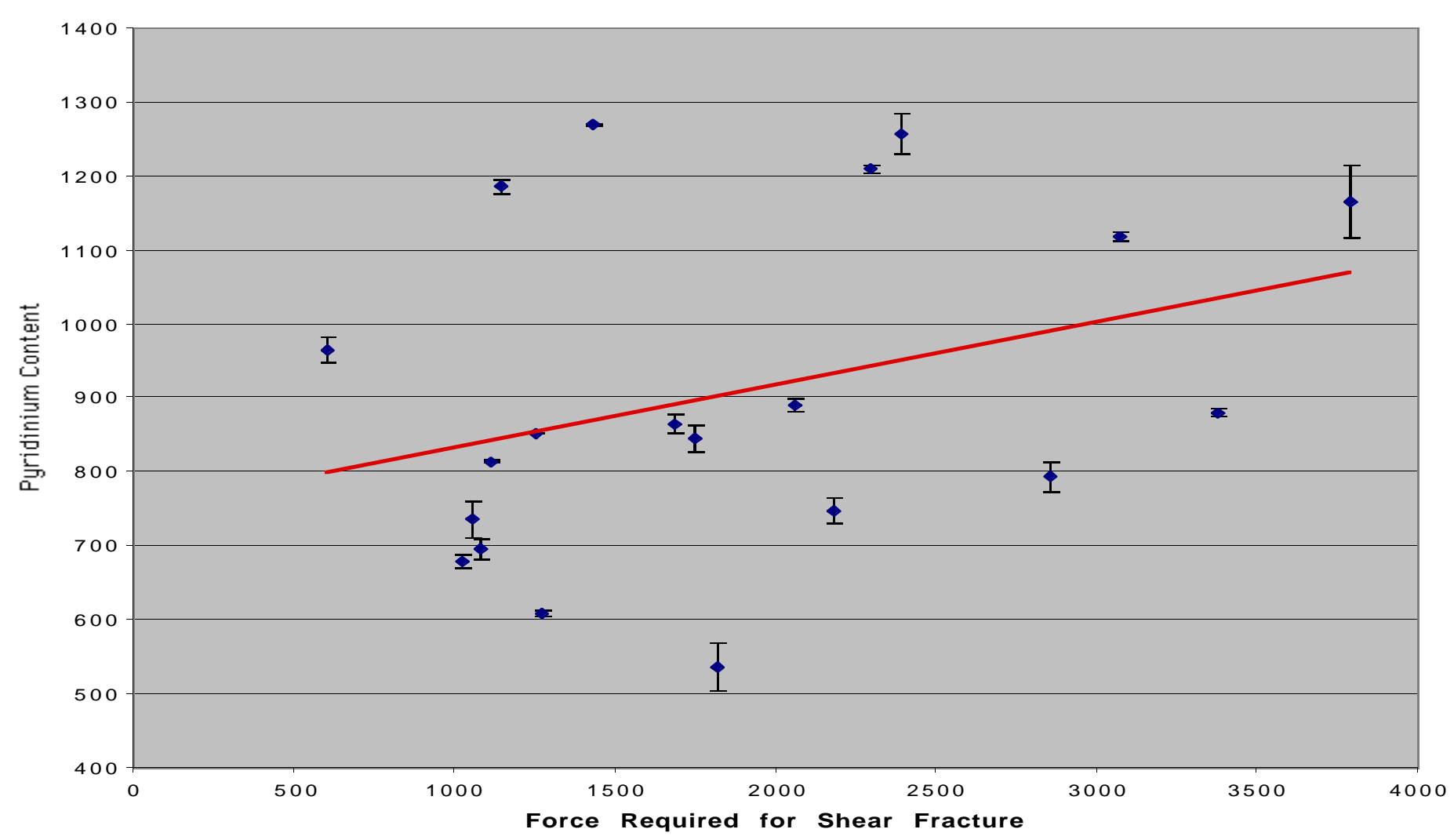

Graph 8. Pyridinium content ( $\mathrm{nmol} / 10.0 \mathrm{mgs}$ bone) showed no correlation to force required for shear fracture in all samples $(\mathrm{p}=0.15 ; \mathrm{N}=20)$. 


\section{Pyridinium Content verse Shear Fracture by Gender}

\section{Graph 9-A}

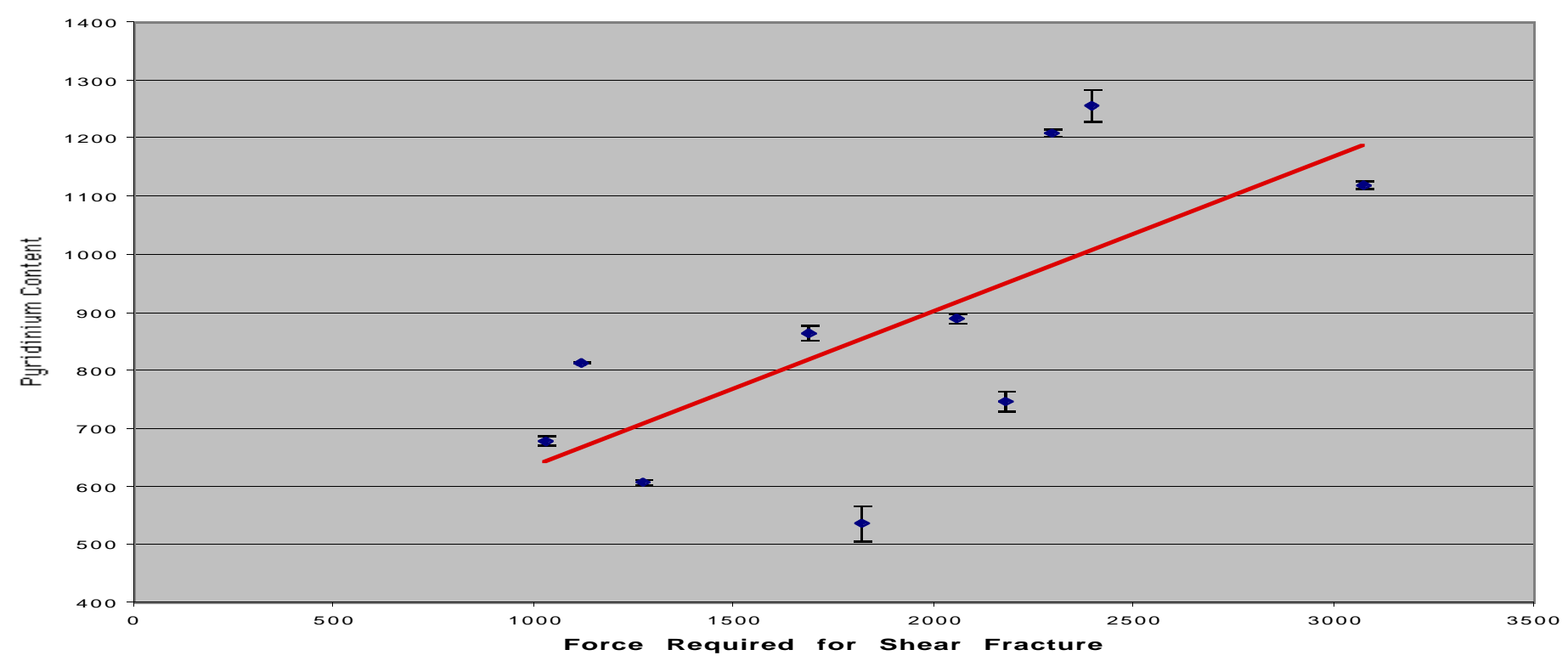

\section{Graph 9-B}

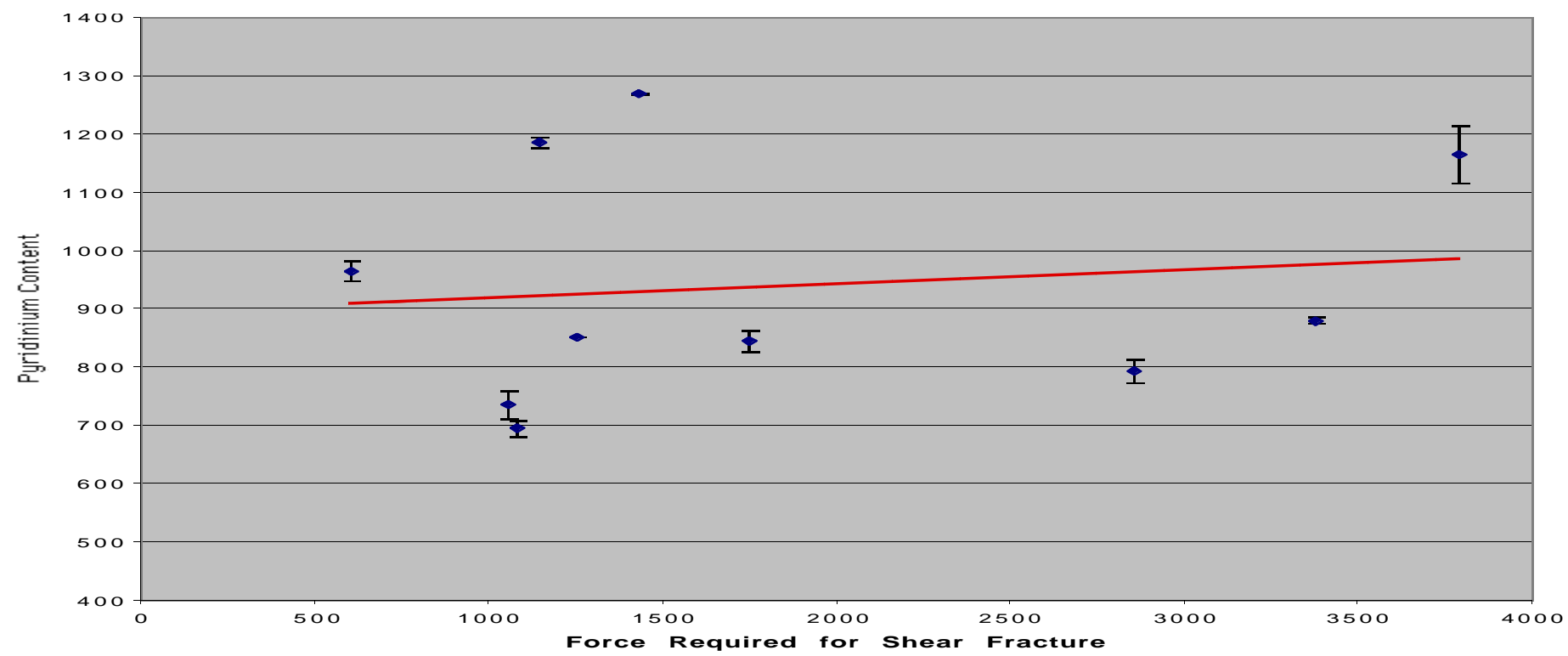

Graph 9-A. Pyridinium content ( $\mathrm{nmol} / 10.0 \mathrm{mgs}$ bone) predicts fracture resistance in the males sub-group $(\mathrm{p}=0.029 ; \mathrm{N}=10)$, but not in the female subgroup $($ Graph 9-B) $(\mathrm{p}=0.71 ; \mathrm{N}=10)$ 


\section{Pyridinium Content by Sex}

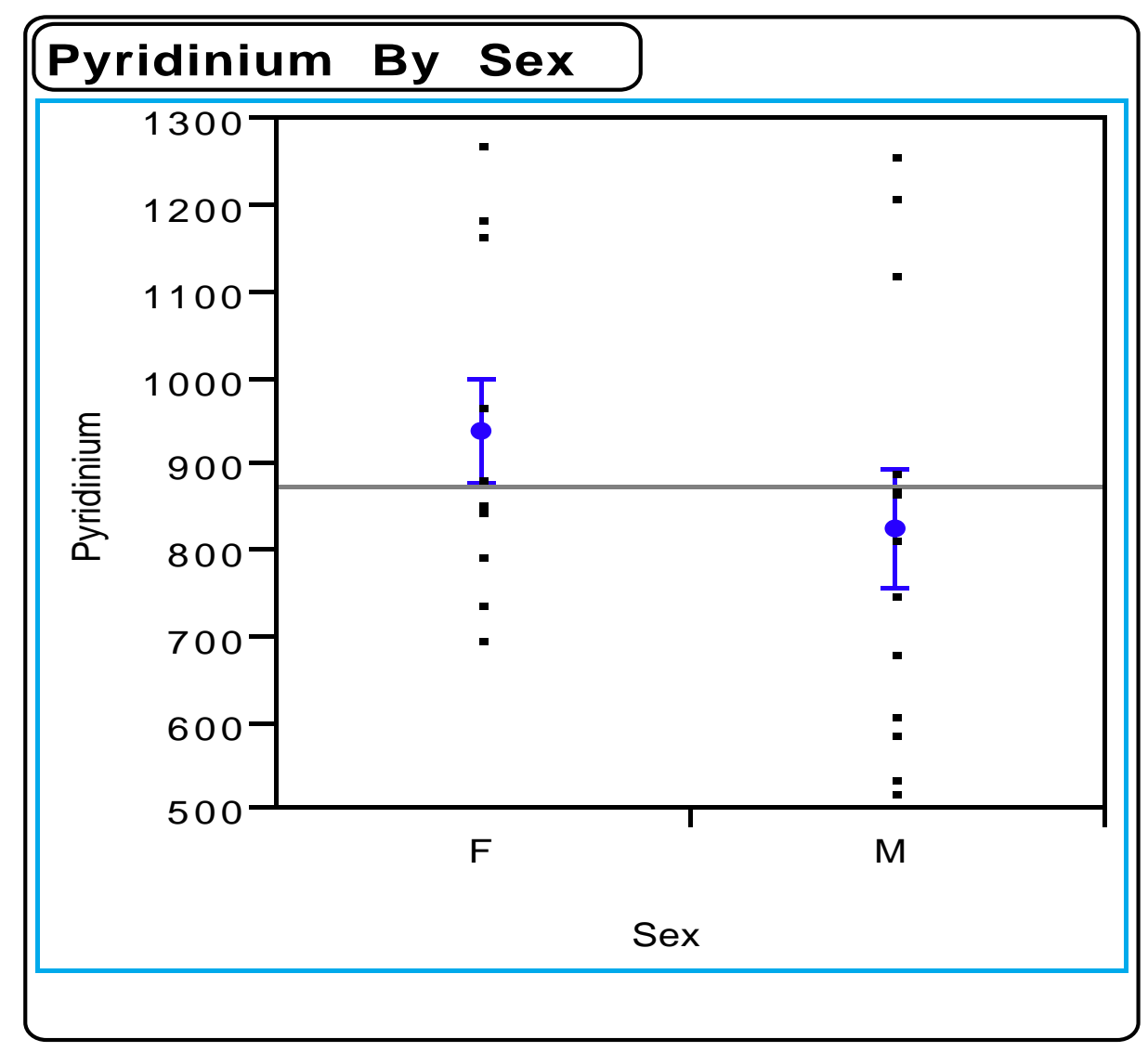

Graph 10. Pyridinium crosslink content (nmol/ $10.0 \mathrm{mgs}$ bone) showed no significant correlation to the sex of the donor $(\mathrm{p}=0.24 ; \mathrm{N}$ $=23$ ). 


\section{Pyridinium Content by Location on Femur}

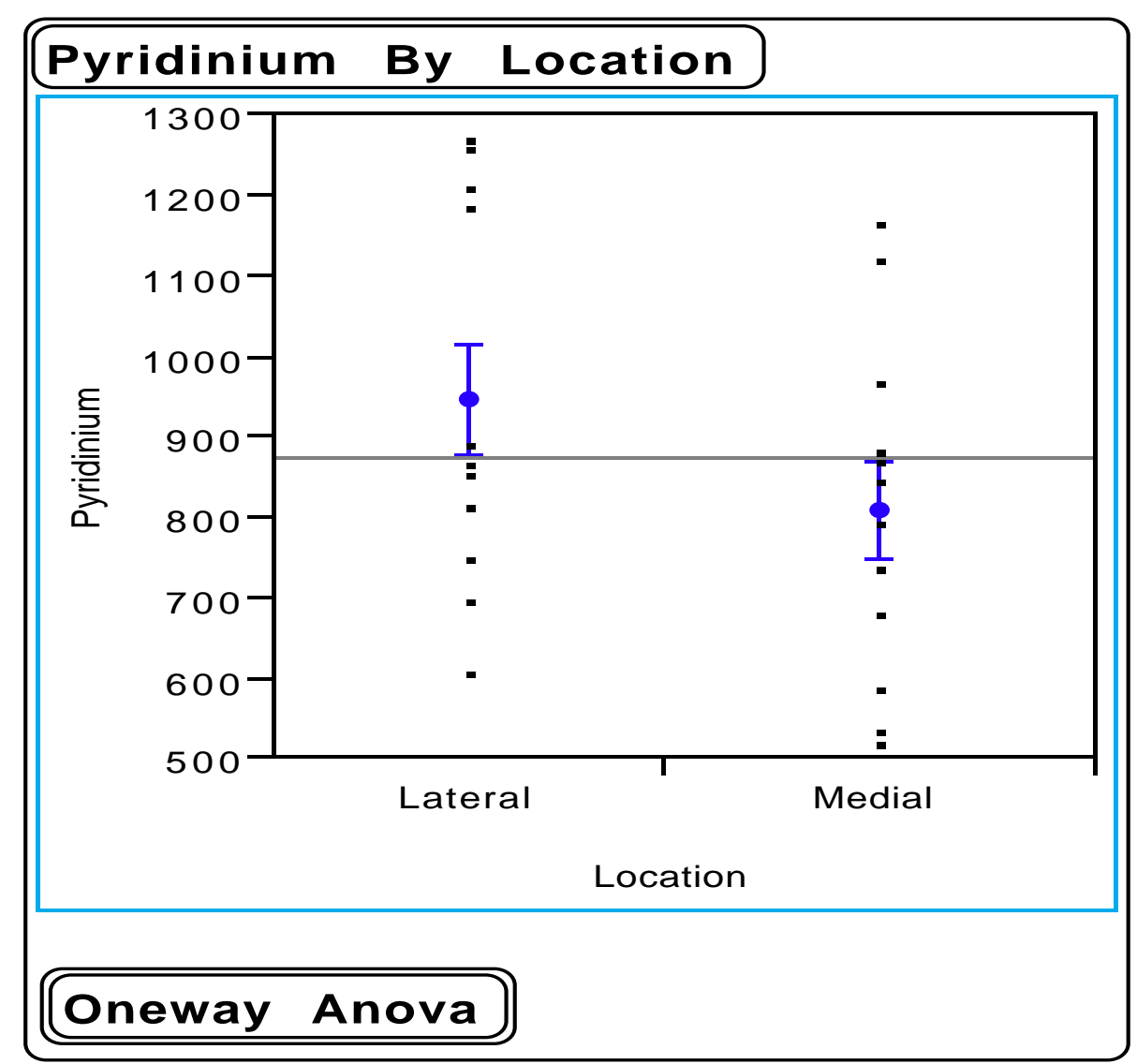

Graph 11. The location of the bone sample from the femur did not correlate to pyridinium crosslink content $(\mathrm{p}=0.16 ; \mathrm{N}=23)$. The anterior site had a significantly lower pyridinium content (nmol/ 10.0 mgs bone) (data not shown). 


\section{Bone Fracture by Location on Femur}

\section{Graph 12-A}

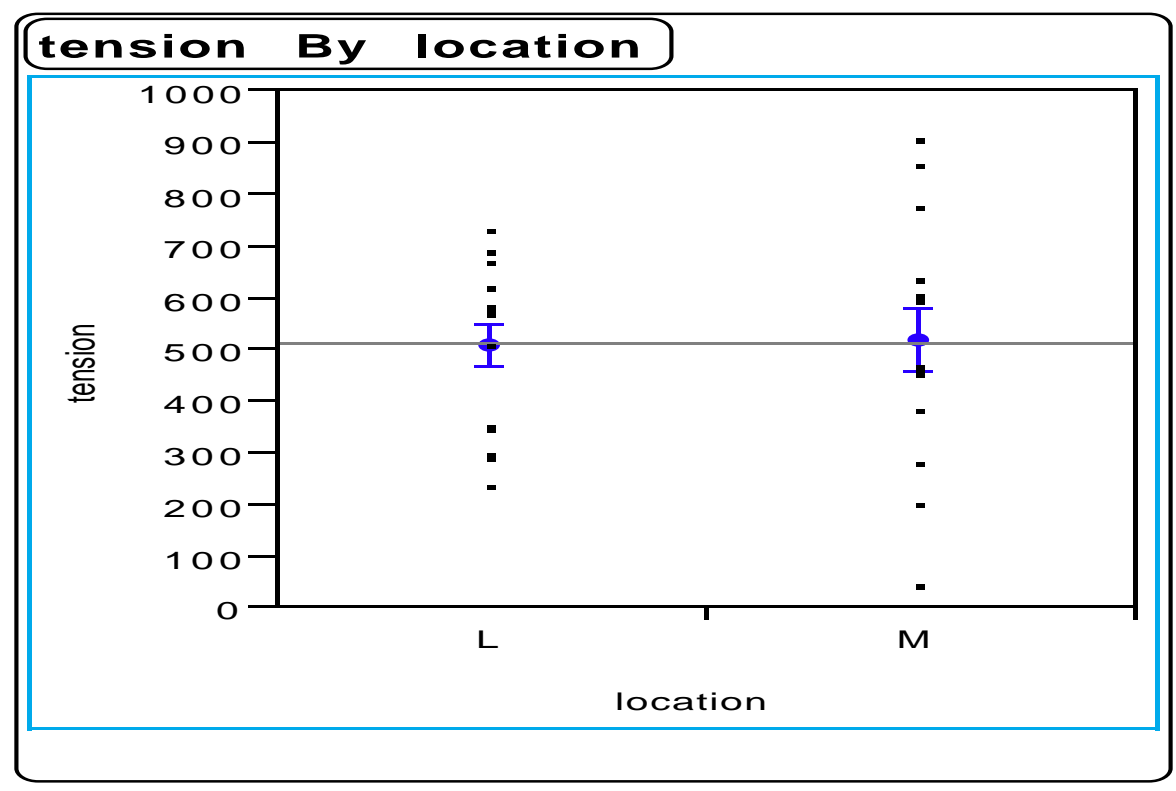

\section{Graph 12-B}

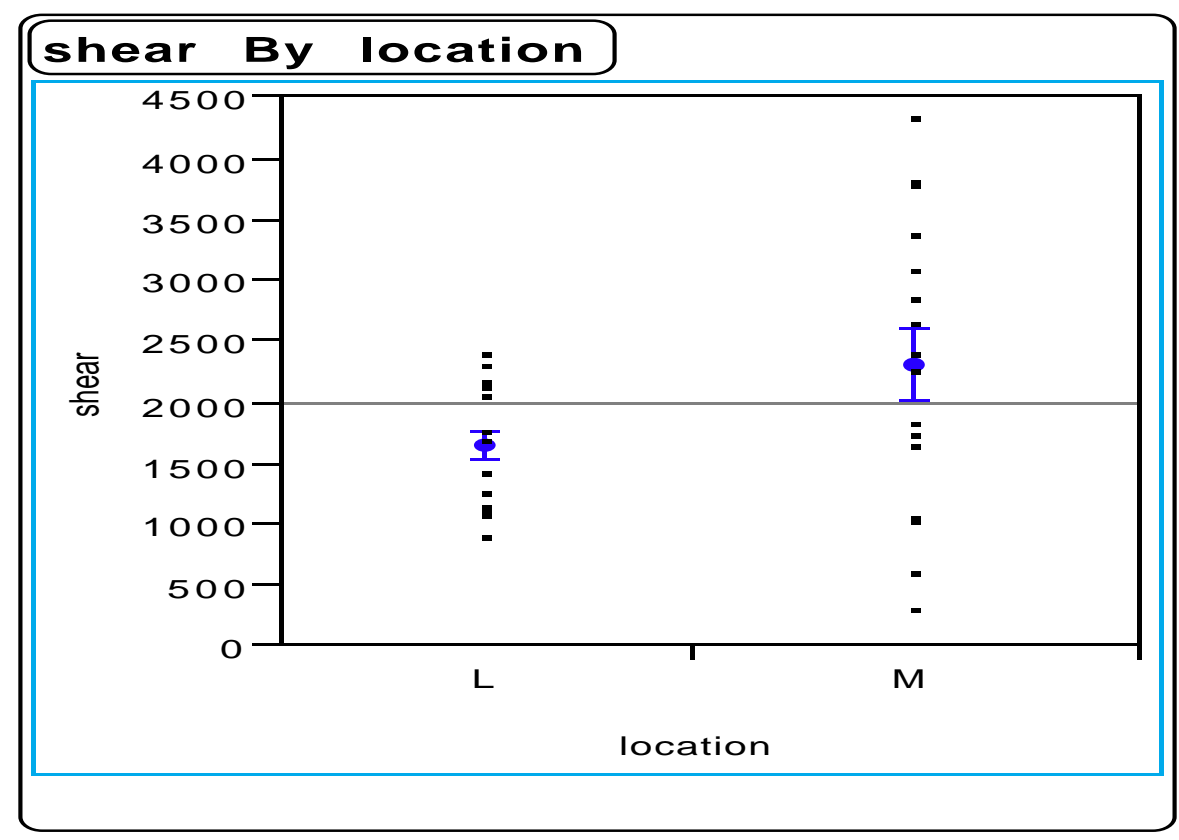

Graph 12-A. Location of bone sample had no effect on tension fracturing. However, for shear fracture the lateral site had a much lower force requirement for fracture which approached significance $(\mathrm{p}=0.07 ; \mathrm{N}=30)$. The cause for this is unknown. 


\section{Fracture Resistance by Gender}

\section{Graph 13-A}

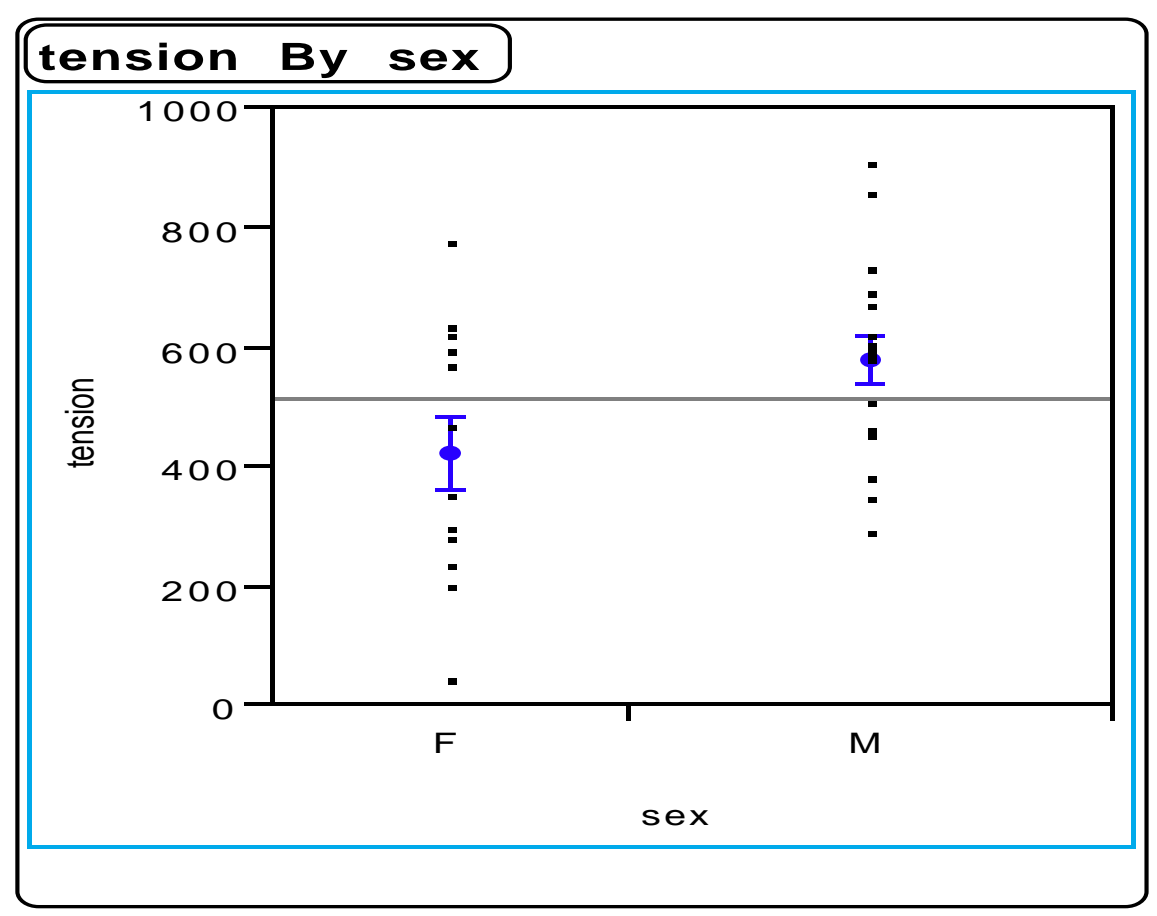

\section{Graph 13-B}

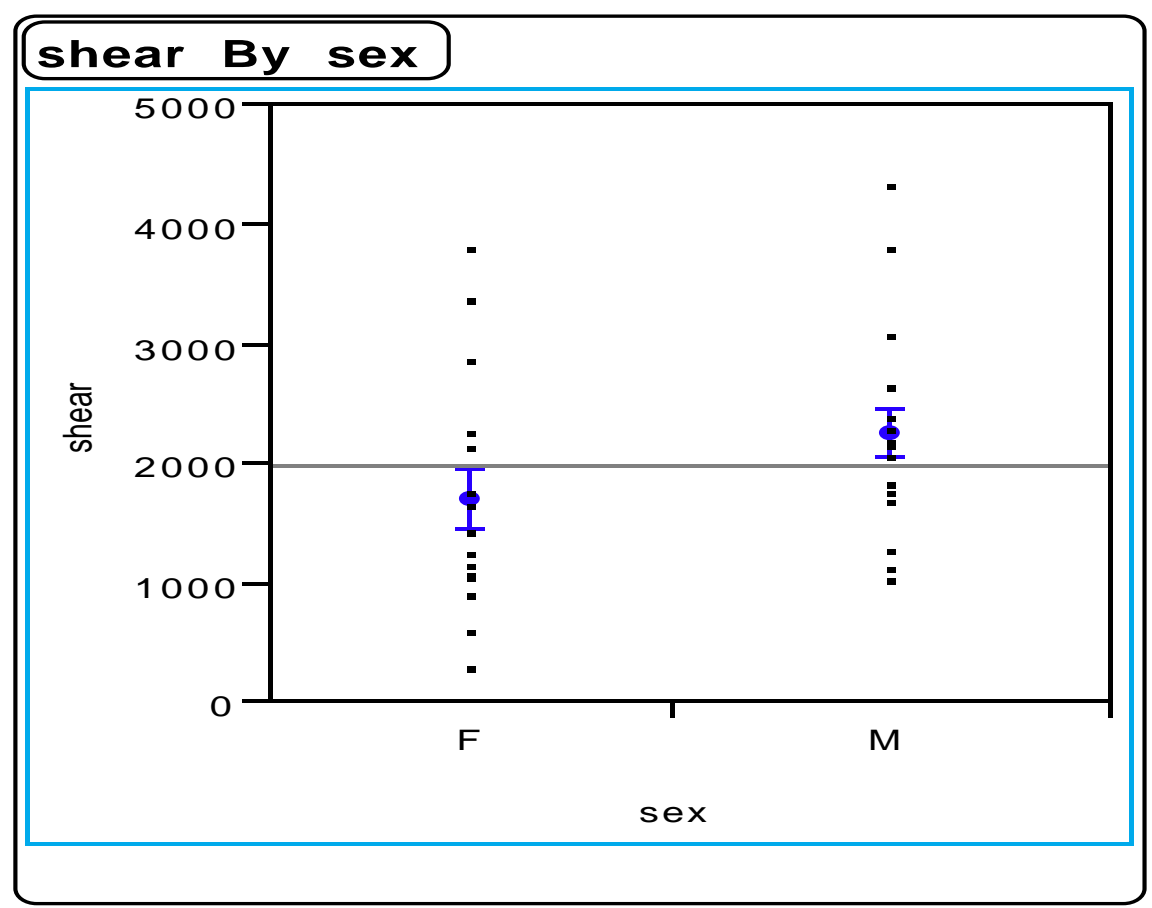

Graph 13-A Males require more force for tension fracture the bone than females $(p=0.04 ; N=28)$. The data did not reach significance when shear fracture was tested $(\mathrm{p}=0.12 ; \mathrm{N}=31)($ Graph 13-B). 


\section{Pyridinium by Age of Donor}

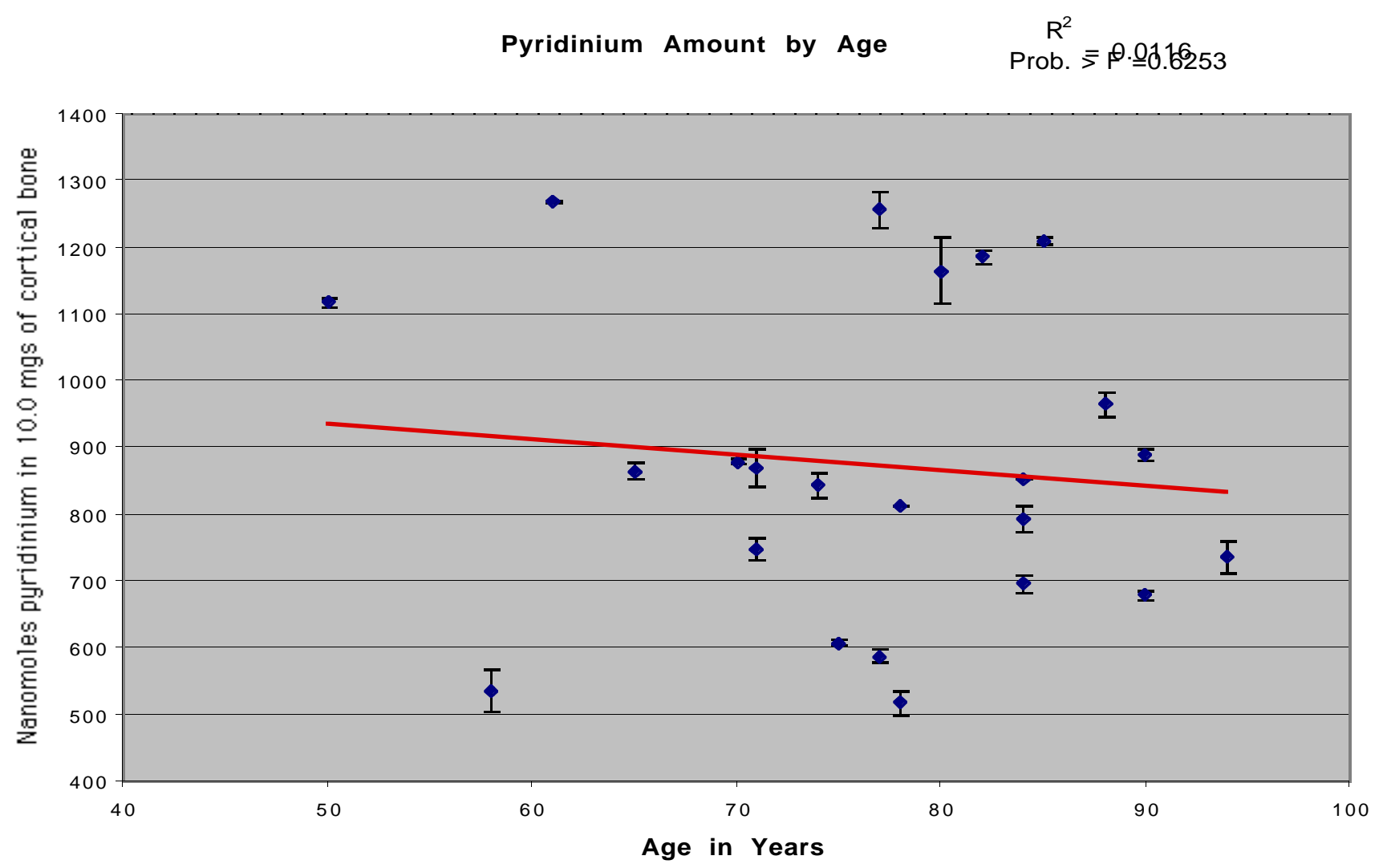

Graph 14. Pyridinium content (nmol/ $10.0 \mathrm{mgs}$ bone) showed no relationship to the age of the donor $(\mathrm{p}=0.63 ; \mathrm{N}=23)$. 


\section{Graph 15-A}

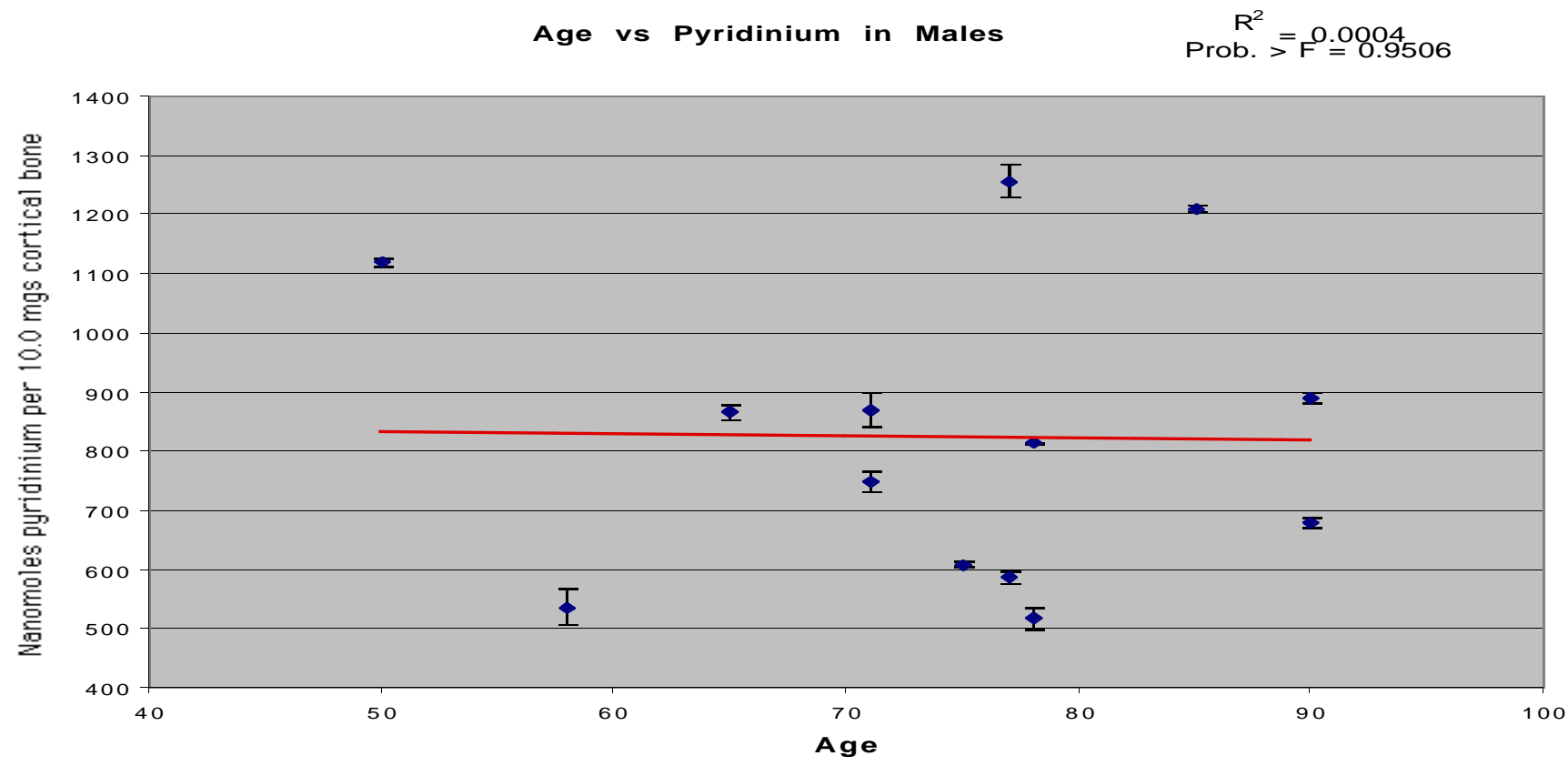

\section{Graph 15-B}

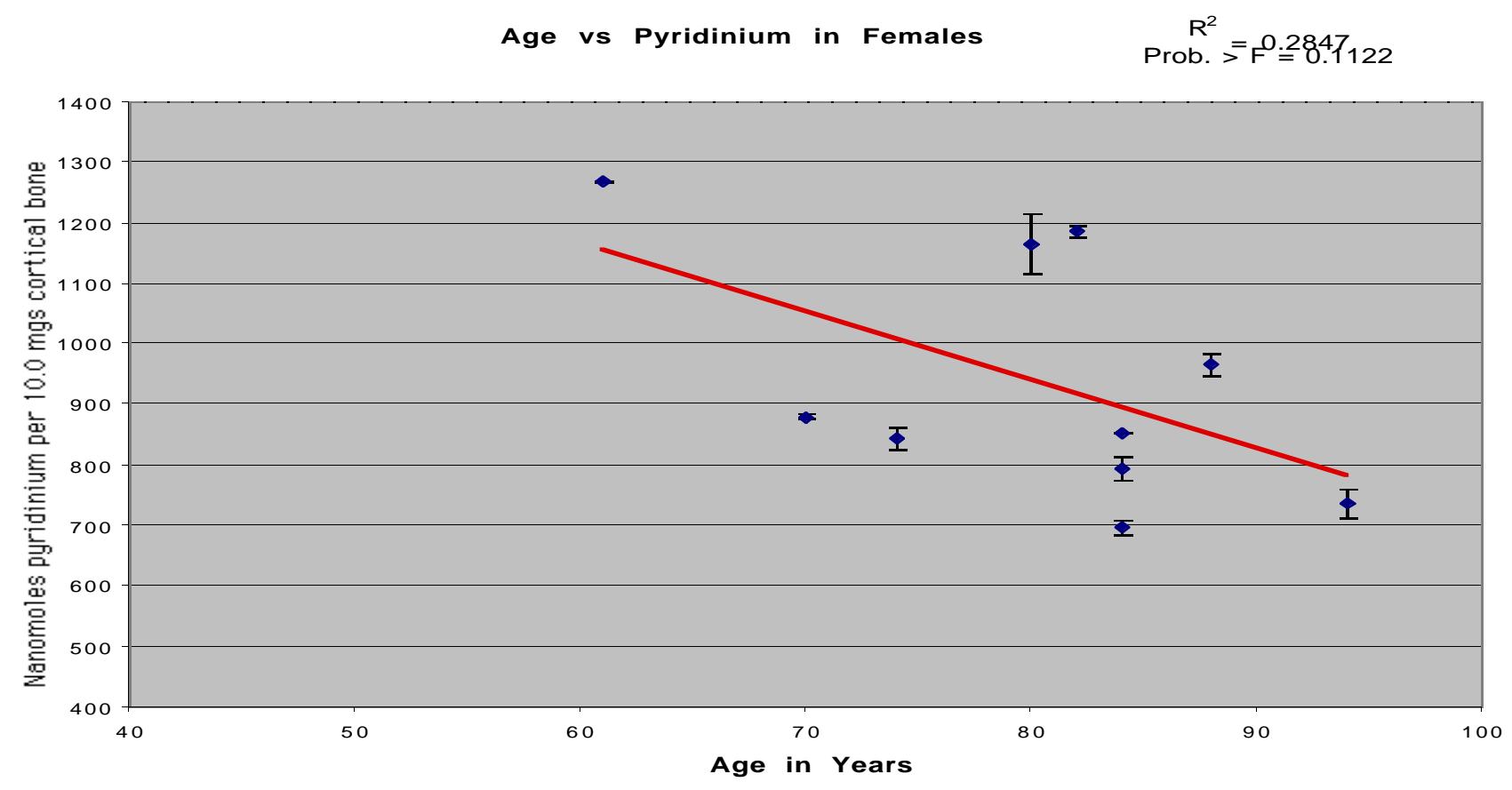

Graph 15-A. Male donors show no decrease in pyridinium content with increasing age. The data from female donors suggest a possible decrease in crosslink content with increasing age, (Graph 15-B), however these data did not reach significance $(p=0.11 ; \mathrm{N}=10)$. 


\section{Collagen Content by Crystallinity and Percent Mineral}

\section{Graph 16}

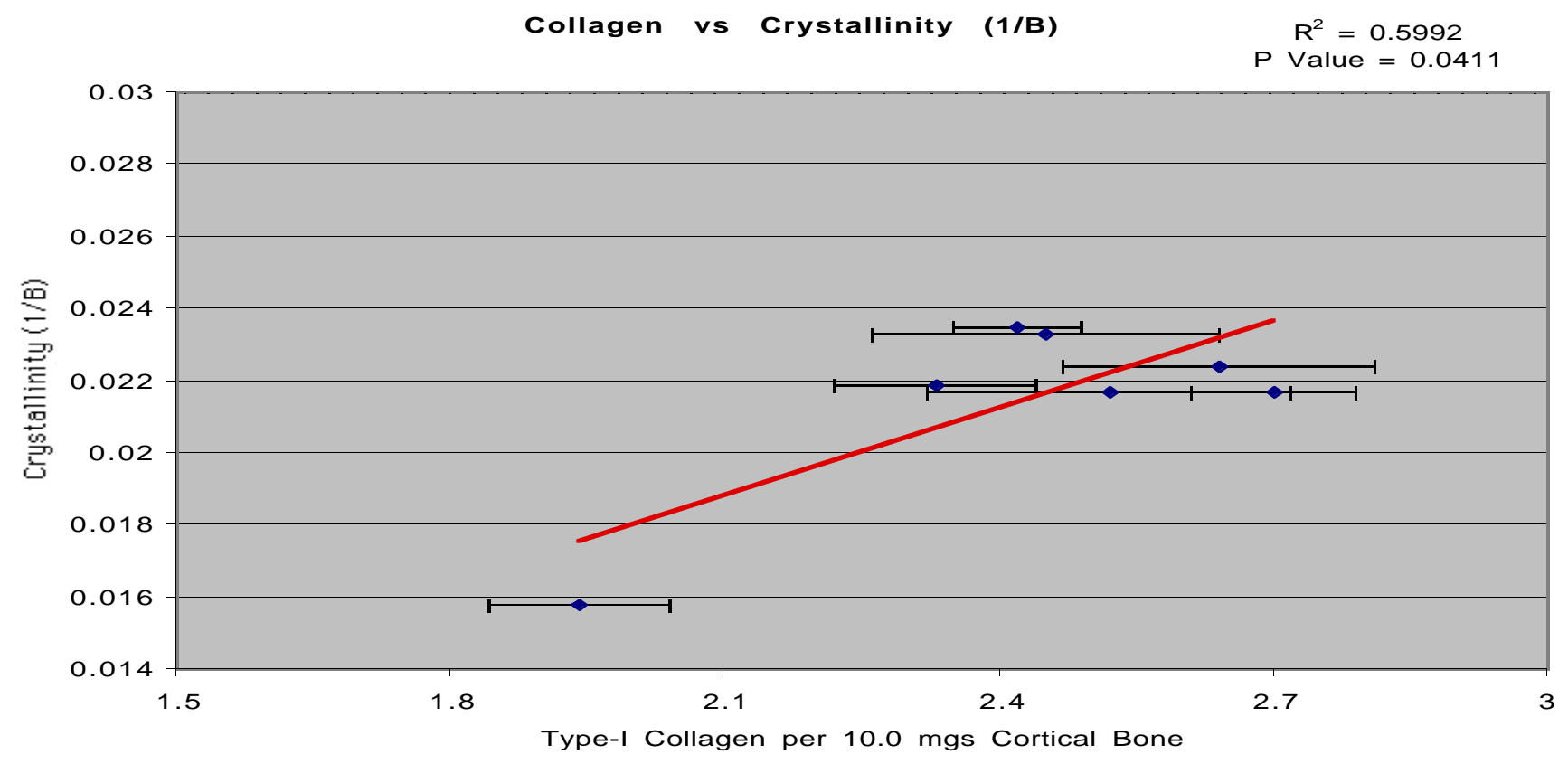

\section{Graph 17}

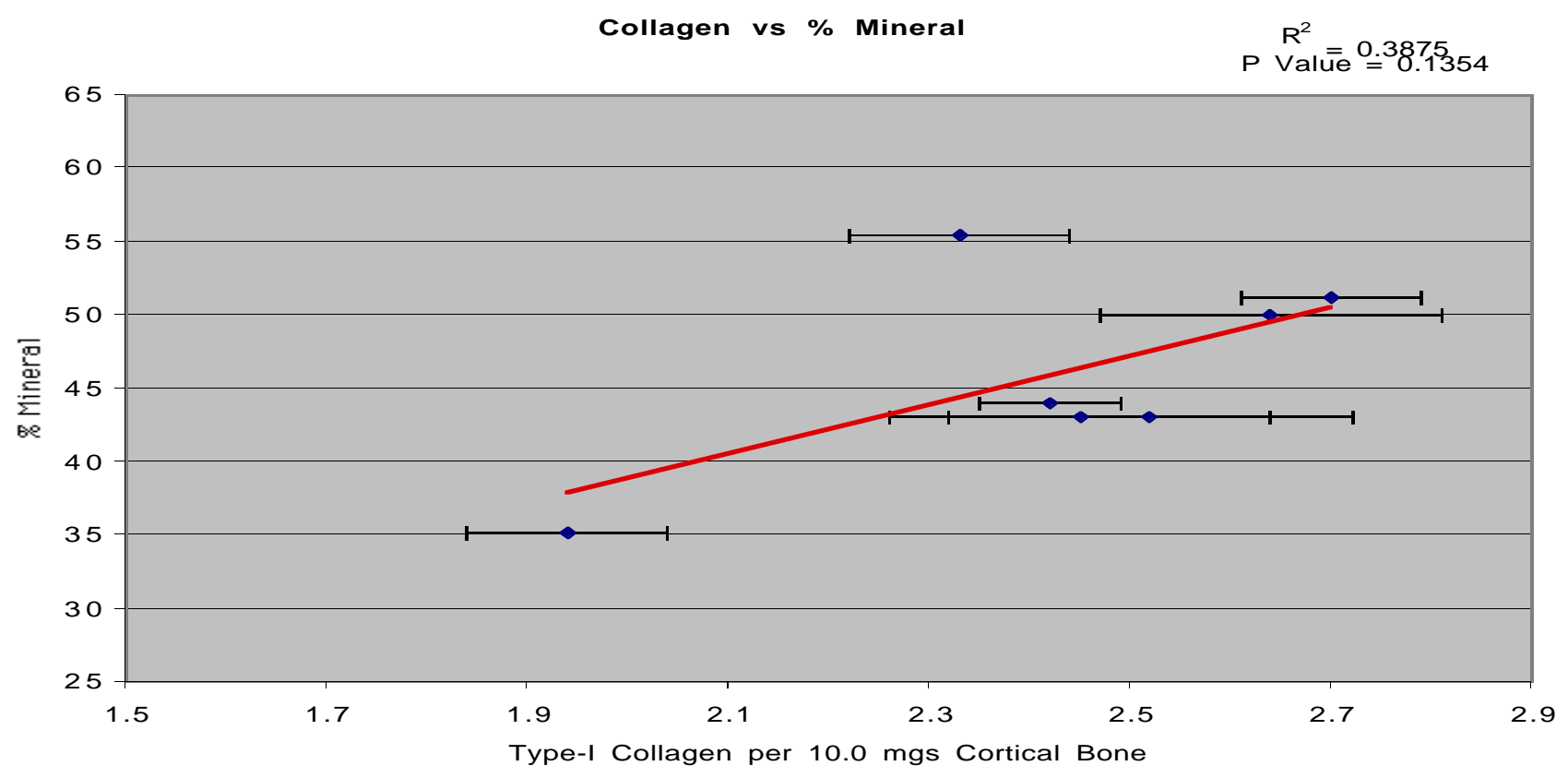

Graph 16. Collagen content showed a significant correlation to crystallinity $(p=.04 ; N=7)$.

Graph 17. Collagen content did not correlate to percent mineral content $(p=0.135 ; N=7)$. 


\section{Pyridinium Content by Crystallinity and Percent Mineral}

\section{Graph 18-A}

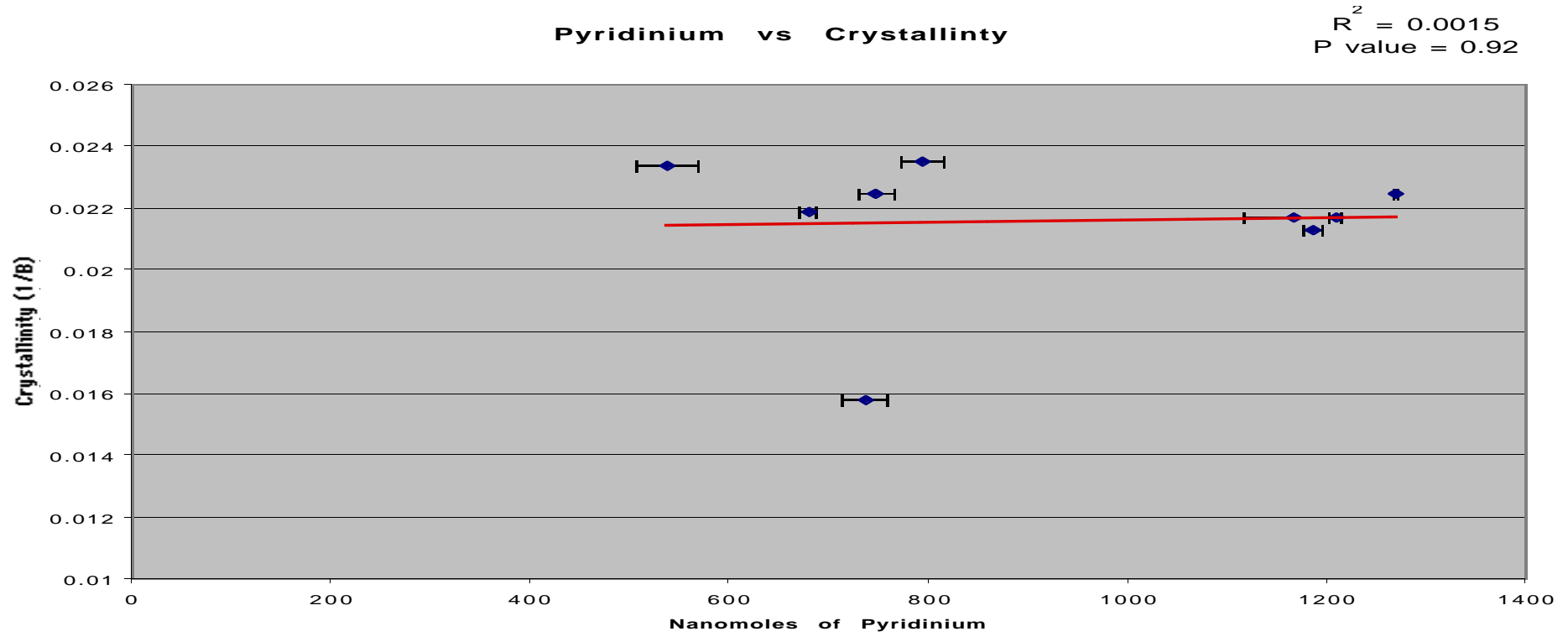

Graph 18-B

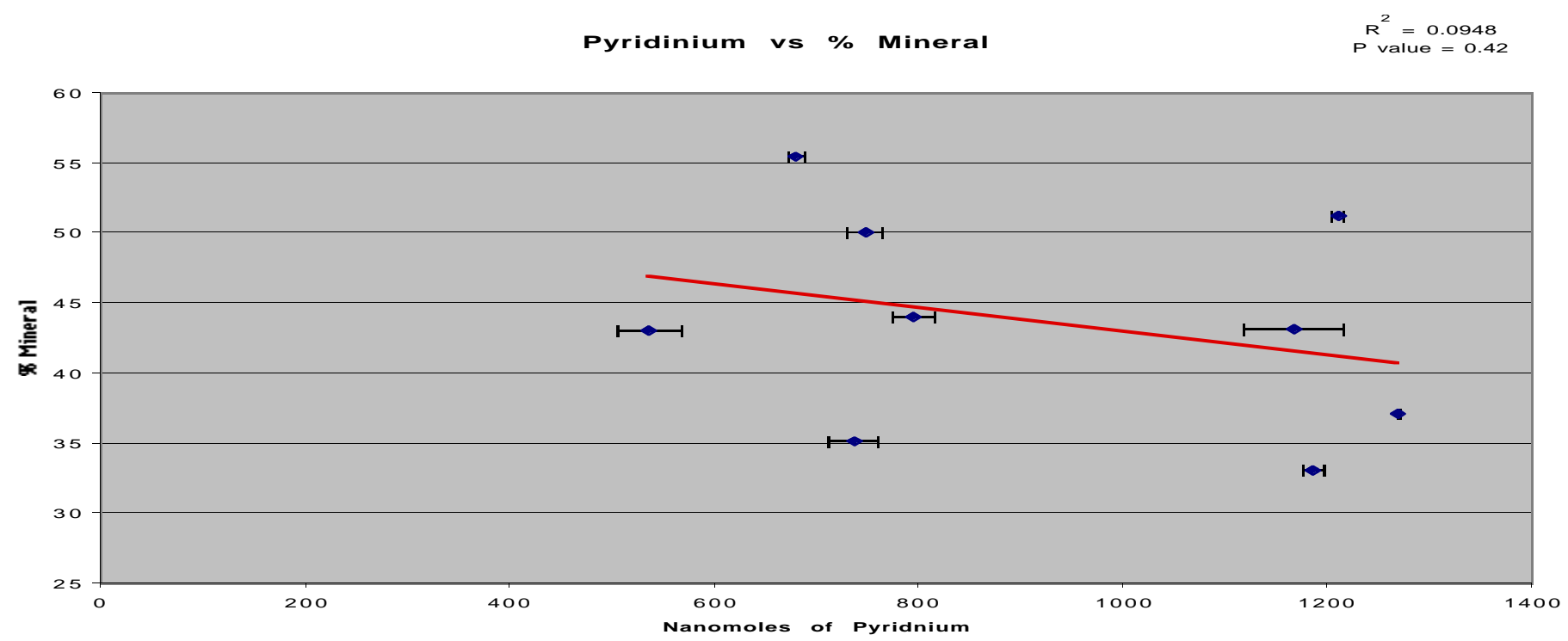

Graph 18-A. Pyridinium content did not correlate to crystallinity $(\mathrm{p}=0.92 ; \mathrm{N}=9)$ nor to percent mineral content $(\mathrm{p}=0.42 ; \mathrm{N}=9)$, (Graph 18-B). 


\section{Pyridinium:Collagen Ratio Effect on Tension and Shear Fracture}

\section{Graph 19-A}

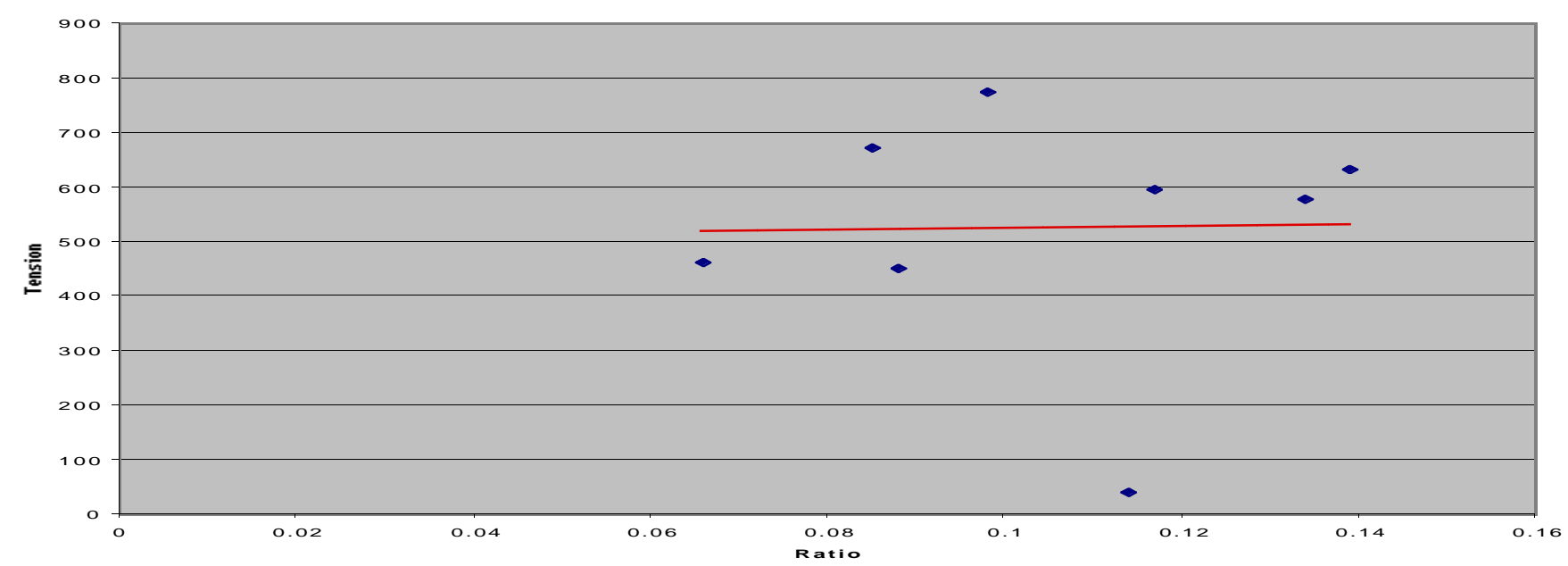

\section{Graph 19-B}

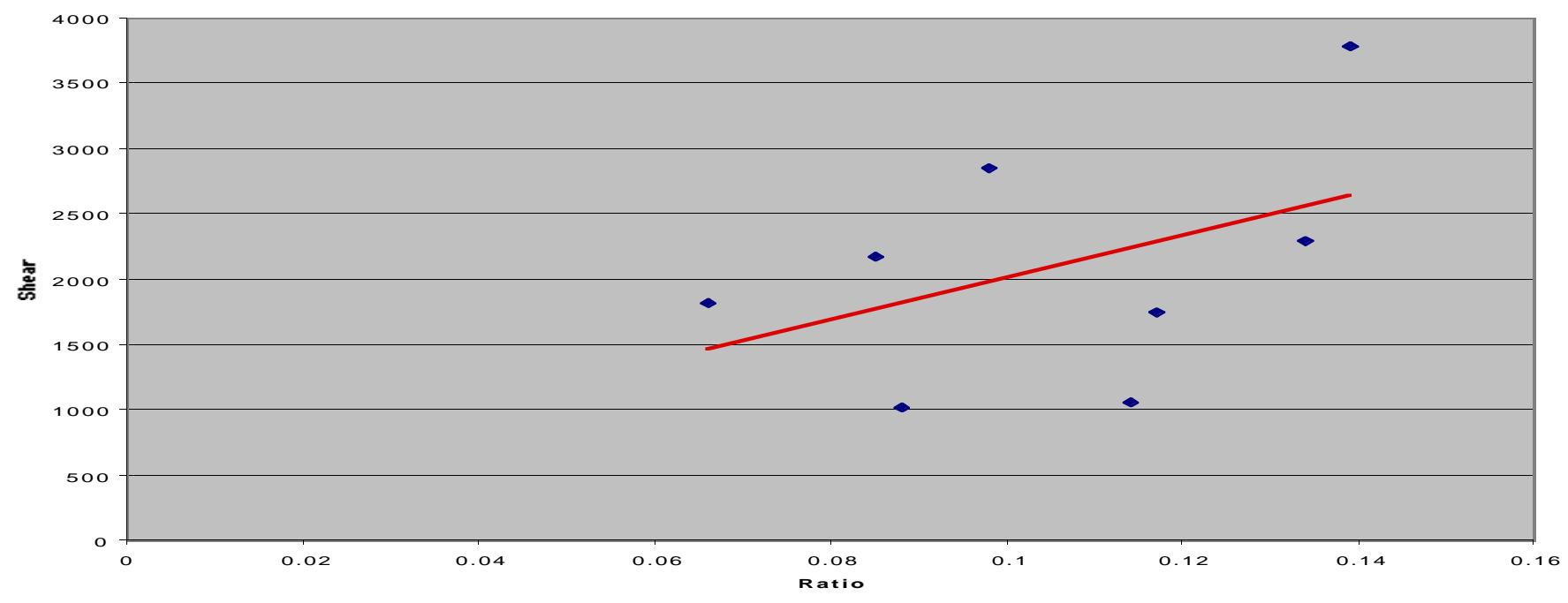

Graph 19-A. The ratio (in moles) of pyridinium to collagen showed no relationship to tension fracture $(\mathrm{p}=0.96 ; \mathrm{N}=8)$ nor to shear fracture $($ Graph 19-B) $(\mathrm{p}=0.27 ; \mathrm{N}=8)$. 


\section{Synergistic Response of Pyridinium and Collagen and their Effect on Tension Fracture}

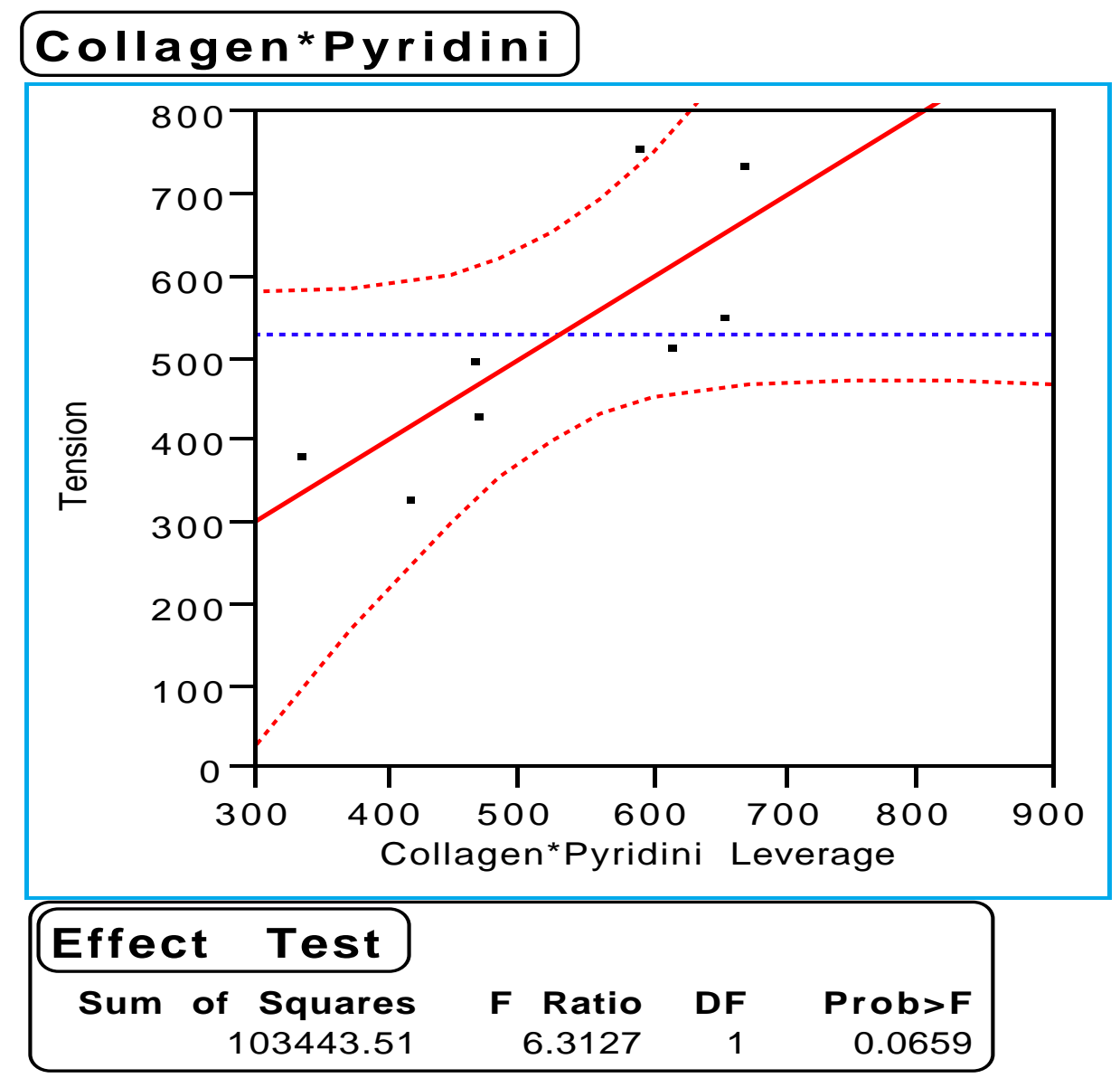

Graph 20. The synergistic response of pyridinium and collagen showed a potential correlation to tension fracture $(\mathrm{p}=0.066 ; \mathrm{N}=8)$. If this can be confirmed it will show that pyridinium does have an effect on collagen and bone strength. 


\section{Pyridinium:Collagen Ratio vs Sex of Donor}

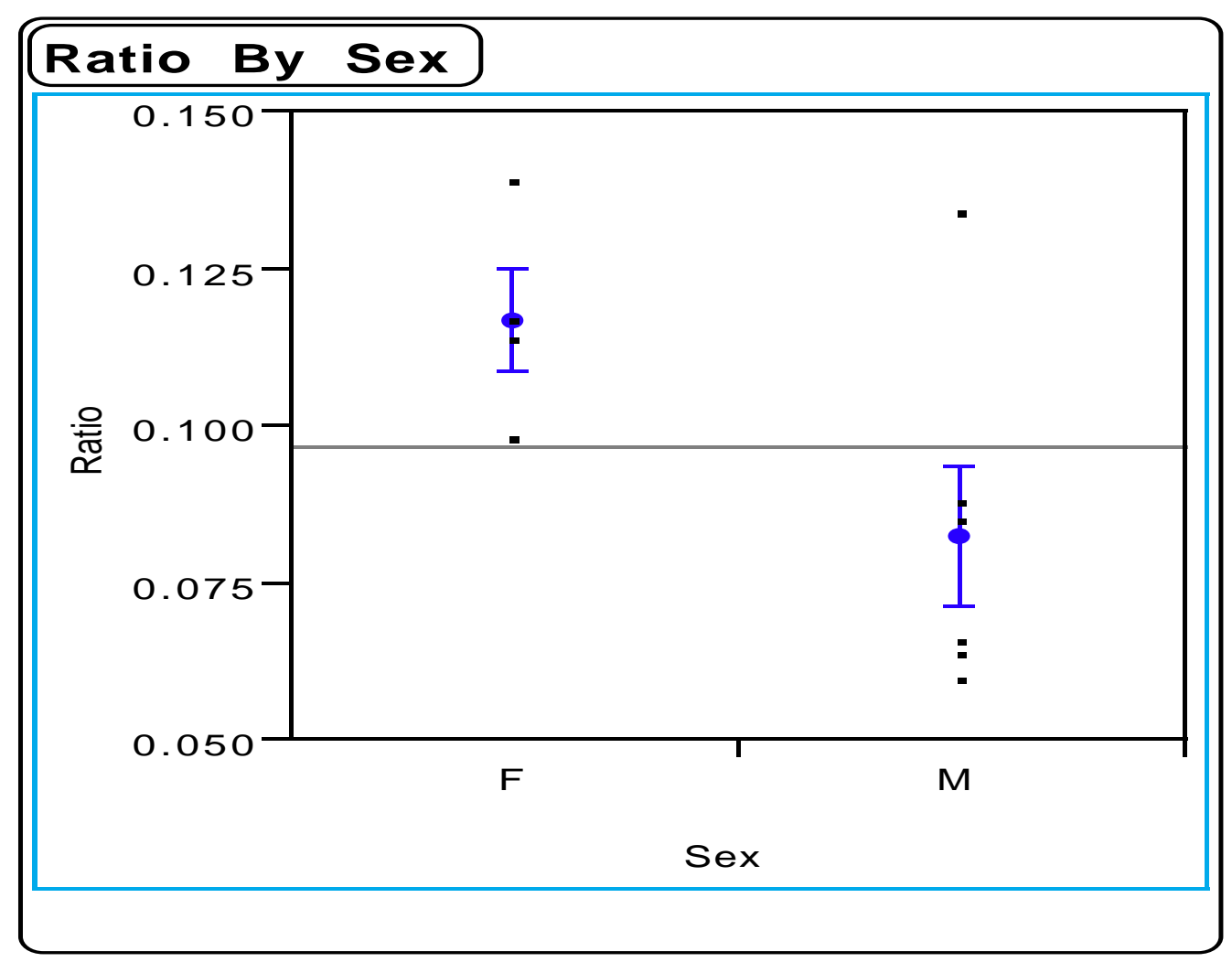

Graph 21. The ratio of pyridinium to collagen (in moles) approached significance ( $\mathrm{p}=0.06 ; \mathrm{N}=10)$. 


\section{Pyridinium:Collagen Ratio vs Age of Donor}

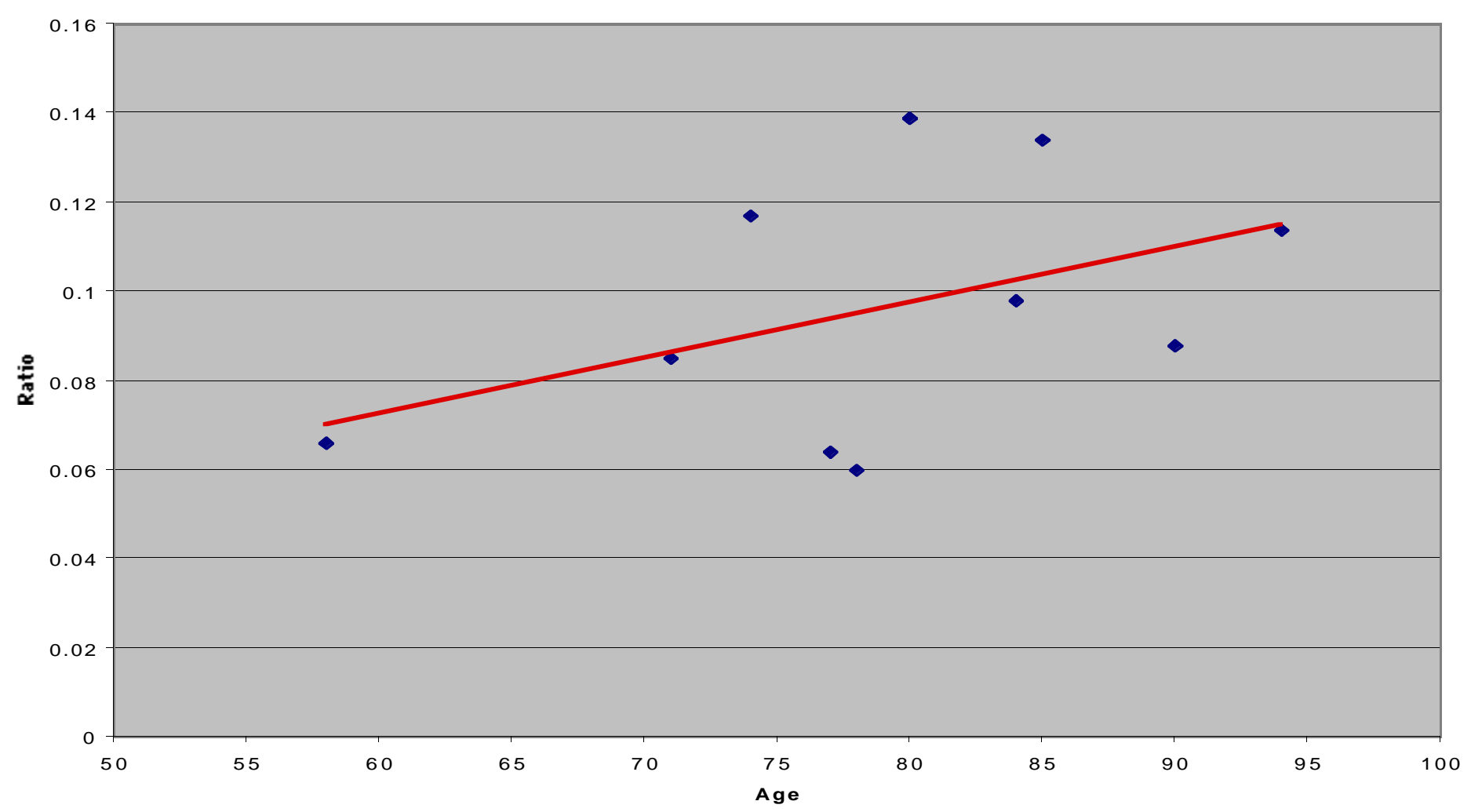

Graph 22. The ratio of pyridinium to collagen (in moles) had no relationship to the age of the donor $(\mathrm{p}=0.2 ; \mathrm{N}=10)$. 


\section{Discussion}

To effectively treat osteoporosis, the components that contribute the most to bone strength must be identified so that therapies can better target them. This study, which compliments work previously reported by Yeni, examined potential relationships between type-I collagen content and the amount of pyridinium crosslinks of collagen to bone's ability to resist fractures (72). Yeni analyzed osteon size and number, bone mineral content and quality, microdamage to bone, and the porosity of bone in an attempt to identify the important biological and morphological contributions of cortical bone to fracture resistance. These combined studies are expected to provide a better understanding of these parameter's contributions to bone's overall strength.

The amount of type-I collagen had a significant, direct relationship to tension fracture $(\mathrm{p}=0.046 ; \mathrm{N}=8)$. An estimated $90 \%$ of the protein infrastructure of bone is comprised of type-I collagen. This collagen-based infrastructure forms the framework upon which non-collagenous proteins are fixed and mineral crystals are deposited and grow. A lower percentage of collagen makes for a leaner scaffold upon which mineral crystals can be deposited, which could cause bone to have a lower bone mineral density (BMD). This defines osteopenia, representing a weakened, low BMD bone. Once a fracture occurs, this bone is termed osteoporotic. Since bone is a composite material its strength is derived from the combined properties of the protein matrix and the associated hydroxyapatite mineral. The collagen fiber framework is not completely rigid, but allows some bending and flexing which accommodates the deposition and growth of hydroxyapatite crystals. The mineral crystals normally elongate parallel to the direction of the fibers. As the crystals increase in length and width, they push against, distort, and 
embed themselves into the collagen fibers. Once the fibrous scaffold becomes mineralized, the bending and flexing of the framework is limited. In cortical bone, available space appears to be a controlling factor in mineral crystal growth. Larger hydroxyapatite crystals have been found to be more brittle than smaller crystals $(11,28,78)$. A lower amount of collagen allows more space for the mineral crystals to grow. If a low collagen content caused a lower BMD with larger, more brittle hydroxyapatite crystals the bone would be predisposed to fracture. This weakening of bone was seen in the results for tension fracture, where bone with less collagen content required less force to fracture. However, even though a significant relationship was seen, due to the relatively low number of test subjects examined, this data may yet be considered preliminary. A cautious view is appropriate since one sample from a 94-year-old donor had a much lower content of collagen than did the other specimens measured, along with the lowest amount of force required for tension fracture.

When collagen content and pyridinium amount were compared together against tension fracture, the data are suggestive of a relationship that might be confirmed through extended studies, $(\mathrm{p}=0.066 ; \mathrm{N}=8)$. The pyridinium crosslinks are thought to aid in the packing of collagen monomers into fibrils and orientating fibrils into fibers. If a relationship between collagen and pyridinium to tension fracture should be confirmed, it will indicate that pyridinium content of bone plays a role in bone strength, possibly through modulating the proper formation and correct orientation of collagen fibers in bone.

Collagen content alone did not predict shear fracture resistance $(\mathrm{p}=0.13 ; \mathrm{N}=8)$. When collagen content and pyridinium amount were compared together against shear 
fracture, the data did not predict bone strength although the data may suggest a potential relationship ( $\mathrm{p}=0.126 ; \mathrm{N}=8)$. Further study may show a significant relationship to shear fracture resistance. This putative relationship may relate to the type of fracture assessed, since the forces used in shear fracture induce a sliding along the collagen fiber length as opposed to the pulling apart of bone which is measured as tension fracture. Since the pyridinium crosslinks connect collagen monomers to each other, and connect collagen fibrils together, perhaps during shear fracture the crosslinks help maintain individual fiber position and oppose the sliding of fibers along one another.

Collagen is vital to bone strength $(10,11,27,33,36,40-42,48)$. Numerous diseases affect collagen, and as a consequence, effect bone and other collagen rich tissues. Osteogenesis Imperfecta (O.I.), first described in the eighteenth century, is one of the most studied collagen-based diseases $(11,27,36,40,41)$. Its severity can vary from mild to lethal in utero. Several mutations in the collagen genes have been identified that cause the many variants of O. I. Mutations that cause shortened procollagen sub-units, homotrimers of collagen, or bent collagen triple helices, are known. Most collagen in O. I. forms thin, abnormal fibers, possibly caused by excessive hydroxylation and glycosylation after translation of the protein. These abnormal fibers produce a malformed bone which the body attempts to remodel, often at an accelerated pace. Although the studies on O. I. have shown collagen's importance to bone formation and its inherent strength, they are all done on genetically abnormal collagen. More recent studies have looked at normal collagen and its effect on bone strength, specifically dealing with osteoporosis. Areas of inquiry have focused on collagen's orientation in bone, collagen's interaction with non-collagenous 
proteins, the association between matrix and mineral, and biochemical changes in collagen as one ages $(2,4,7,9,10,13,24,28,34,48,58)$.

From the present study, it is seen that a decrease in collagen content correlates with a decrease in bone strength. This agrees with several studies that have linked the content of collagen in bone to its resistance to fracture $(11,78)$. Bailey et al. found a strong correlation between collagen content and maximum stress levels, $\left(\sigma_{\max }\right)$, Young's modulus, $(\varepsilon)$ (which is the maximum slope of the stress-strain curve) and the energy absorption level, $\left(\omega_{\mathrm{abs}}\right)$, during mechanical compression (78).

With age, and after the menopause, bone mass decreases. The present study tested whether the percentage of type-I collagen decreased with increased age. A relationship was suggested in the present study, however this putative relationship could not yet be confirmed, $(\mathrm{p}=0.12 ; \mathrm{N}=13)$. This trend approached significance even though donors that took antiresorptive agents to aid in arresting bone loss were not excluded from the study and were not identified for these studies. This could be important because the age of the donors showed a very strong correlation to fracture resistance (tension fracture $\mathrm{p}=0.037 ; \mathrm{N}=28$; shear fracture $\mathrm{p}=0.006 ; \mathrm{N}=31$ ) (72). Age, and its likely effects on collagen content and overall bone mass, apparently correlates with bone strength. However, when age and collagen content together were compared against tension or shear fractures, no significant relationship could be demonstrated. This was unexpected and the low sample number assessed may have compromised this outcome. Since collagen amount seems to predict bone strength, as does age, together they were expected to show an even stronger correlation to bone strength. Bone's decrease in collagen content with respect to age agrees with several related studies that 
have shown similar results $(76,77,78)$. This decrease in collagen content may be due to the imbalance in the highly coupled process of resorption and formation. Beginning at around age 40 for cortical bone, a slow age-dependant loss of bone occurs. This loss of bone is believed to be a result of normal resorption, with a failure of the osteoblasts to completely refill the resorption pits. Little is known about the decrease in efficiency of the osteoblasts, though a decrease in quality and quantity of type-I collagen production is a distinct possibility. Coupled with the postmenopausal phase of rapid bone loss due to an increase in the rate of bone resorption, and the reason for the age related decrease in bone strength in women seems evident.

Significant results indicating sex-linked characteristics were obtained for type-I collagen content $(\mathrm{p}=0.038 ; \mathrm{N}=13)$. Males averaged 2.61 milligrams \pm 0.08 of collagen per 10.0 milligrams of cortical bone (average age 75), while women had a lower collagen quantity, 2.32 milligrams \pm 0.09 (average age 85 ). However these data should be considered preliminary due, again, to the low number of test subjects and the possibility that age contributed to the effect. As to the sexually dimorphic differences in collagen content of bone, there are known differences in the skeletal system between men and women, and it seems likely that collagen production could have differences also. Barring disease, males typically attain a higher peak bone mass than do females, and females, after the menopause, lose bone at a faster rate than males (17). The linear growth of bone continues till about the age of twenty, while its radial growth continues until age thirty. Growth is then followed by a slow, continual decrease in bone mass. It is estimated that the age dependant phase of bone loss begins at age thirty-five for trabecular bone, and at age forty for cortical bone, and that this bone loss continues up until death (14). It is 
believed that men and women lose similar amounts of bone during this phase. This loss has been estimated to be about twenty-five percent of both trabecular and cortical bone over a lifetime (14). Women, however, go through another rapid phase of bone loss that begins at the onset of menopause. This phase of bone loss can be ameliorated by estrogen replacement therapy. In the absence of therapy an additional ten percent loss of cortical bone and an additional twenty-five percent loss of trabecular bone in women may occur $(14,29)$. Thus, the issues raise the question of whether the differences in collagen content between males and females are an artifact due to the average age of donors sampled, or might accurately reflect a limited collagen production by females in attaining peak bone mass, or be a consequence of the increase in bone resorption in postmenopausal females which is followed by the formation of new bone whose quality and quantity may be compromised. Donor sex also had a significant relationship to tension fracture $(\mathrm{p}=0.042 ; \mathrm{N}=31)$, perhaps due to collagen content.

When pyridinium content was compared to sex, no trend was apparent $(\mathrm{p}=0.24$; $\mathrm{N}=23$ ). The female samples averaged 940 nanomoles $\pm 72 \mathrm{~nm}$ of pyridinium per 10.0 milligrams of cortical bone, giving a molar ratio of pyridinium to collagen of 0.12 . Male donors averaged 823 nanomoles $\pm 64 \mathrm{~nm}$ of pyridinium for a molar ratio of 0.08 . When taken as a molar ratio, women appear to have a more highly cross-linked collagen scaffold, with one half more pyridinium crosslinks per mole of collagen, than did men. This difference approached statistical significance $(\mathrm{p}=0.06 ; \mathrm{N}=10)$. A larger sample size may provide the data to make a definite conclusion. Although both men and women in this study have a lower molar ratio of pyridinium to collagen than that which has been previously reported, this may be due to the possible degradation of pyridinium during the 
acid hydrolysis of bone and the lack of valid comparisons between EIAs and other methods that have been used for detection $(61,75)$. Another possibility is that there is a loss of pyridinium crosslinks as a consequence of a loss of mature bone with age resulting in a decreased ratio of pyridinium to collagen.

No significant relationship was observed when comparing the pyridinium to collagen ratio against tension fracture $(\mathrm{p}=0.96 ; \mathrm{N}=8)$. When relating this ratio to shear fracture, again no significance was observed $(\mathrm{p}=0.27, \mathrm{~N}=8)$. This was surprising since it was expected that pyridinium crosslinks would help orientate and stabilize the collagen fibers, and thus strengthen bone. Also the synergistic effect of collagen and pyridinium on both tension and shear fracture approached significance. Relating the molar ratio of pyridinium to collagen to the age of the donors showed no significant correlation.

It was assumed that there would be no biochemical differences in collagen with respect to the site from which bone was removed, and indeed the non-reducible crosslink content did not statistically differ between the lateral site and medial site of the femur shaft $(\mathrm{p}=0.16 ; \mathrm{N}=23)$. When all samples of pyridinium were related to the age of the donors, no correlation was observed $(\mathrm{p}=0.625, \mathrm{~N}=23)$. Interestingly, when dividing the donors by sex, age may effect pyridinium content in females, although this effect could not be shown to be significant in the present study $(\mathrm{p}=0.11 ; \mathrm{N}=10)$. This trend was not evident in males $(\mathrm{p}=0.95 ; \mathrm{N}=13)$.

If the decrease in mature pyridinium crosslinks in females could have been demonstrated, such results would disagree with several studies that have found no change in pyridinium levels as age increases (48). However, not all studies separated the donors according to gender. An age related decrease in mature crosslinks might be a reasonable 
expectation due to the increased resorption rate seen in post-menopausal females. A decrease in older, more mature bone would correlate to a decrease in mature nonreducible pyridinium crosslinks.

When relating pyridinium content to tension fracture, no significant correlation could be attributed when all samples were pooled $(\mathrm{p}=0.32 ; \mathrm{N}=19)$. However, a gender specific response was observed. Pyridinium content directly correlated to force required for tension fracture in males $(\mathrm{p}=0.0478, \mathrm{~N}=10)$. This was not seen in female donors ( $\mathrm{p}$ $=0.91 ; \mathrm{N}=9$ ). In looking at shear fracture, similar results were apparent. With samples from both genders pyridinium content did not predict shear fracturing $(\mathrm{p}=0.1526 ; \mathrm{N}=$ 20). The female sub-group showed no significance $(\mathrm{p}=0.71 ; \mathrm{N}=10)$, but pyridinium content did correlate to force required for shear fracture in the males $(\mathrm{p}=0.029 ; \mathrm{N}=10)$. These results appear to follow previous studies on pyridinium content and its lack of effect on bone strength, but also pose new questions concerning possible gender-related differences in the non-reducible collagen crosslinks of bone.

Bailey et al. (48) studied biochemical differences in collagen from the head and neck regions of the human femur in young, old, and osteoporotic bone. They reported that the hydroxylation of lysine was similar between young and old bone, but was much higher in osteoporotic bone. The increase in lysyl hydroxylation was more apparent in the head region of the femur, although both regions were different in the osteoporotic samples. They also compared the reducible crosslink hydroxylysino-5-ketonorleucine (HLKNL) and the non-reducible crosslink, pyridinoline, between normal and osteoporotic bone. The divalent HLKNL was found to be reduced $20 \%$ in the head region, and by $44 \%$ in the neck in osteoporotic bone, while the mature trivalent 
pyridinoline remain the same between young, old, and osteoporotic bone in both the head and neck region of the femur. Bailey et al. had only thirteen donors in their study and sub-division by gender was not done, nor was any analysis of fracture resistance.

Bailey et al. continued with another study in which they found a decrease in collagen content with age (76). They also confirmed earlier studies reporting an increase in hydroxylation of lysine and a decrease in reducible crosslinks in osteoporotic bone. They failed to determine if the increase in lysyl hydroxylation caused a difference in ratio of dihydrolysinonorleucine and hydrolysinonorleucine. They also did not determine if the increase in lysyl hydroxylation changed the ratio of pyridinoline to deoxypyridinoline.

Bätge et al. (24) compared biochemical differences of collagen from trabecular bone of the vertebrae from normal and osteoporotic patient's. Bätge observed an increase in the hydroxylation of lysine on the $\alpha(2)$-I chain of collagen correlated to decreases in the vertebral bone mass and the vertebral trabecular bone volume. The hydroxylation of lysine on the $\alpha(1)$-I chain remained the same for all samples. Similar results were obtained when sub-dividing the samples by gender. No correlation could be attributed between lysyl hydroxylation and the age of the female donors; however differences were found between pre-menopausal donors, post-menopausal donors, and those with established osteoporosis. Bätge does not discuss pyridinium crosslinks, nor how they could be affected by an increase in lysyl hydroxylation on the $\alpha(2)-I$ sub-unit. The ratio of crosslinking appears to be 3.5:1.0 for $\alpha(1)$-I to $\alpha(2)$-I sub-units, so the increase in hydroxylation of the $\alpha(2)$-I sub-unit may not effect cross-linking as much as it would if it 
occurred in the $\alpha(1)$-I sub-units (50). Bätge also looked at trabecular bone from the vertebrae, thus those differences may not apply to cortical bone from the femur.

Oxlund et al. (77) studied trabecular bone from vertebral samples from normal and osteoporotic patients. They found an age-related decrease in collagen density (mg collagen $/ \mathrm{cm}^{3}$ ) but no difference between normal and osteoporotic patients. Both divalent crosslinks were found to be reduced in osteoporotic patients but not the nonreducible trivalent pyridinium crosslinks. This agrees with Bailey and Bätge, and may be explained by the fact that the divalent crosslinks outnumber the mature trivalent crosslinks 2-4 to 1 . Large decreases of these reducible crosslinks could dramatically reduce the strength of the newly formed bone, and thus the strength of the entire bone, without changes in the pyridinium crosslink concentration. Oxlund et al. also state that they found no change in pyridinium content with respect to age, but provide no data.

Lo Cascio et al. (5) found an increase in urinary GHYLin post-menopausal osteoporotic women compared to post-menopausal normal women. The increase in urinary GHYL may be a result of remodeling at the fracture sites as opposed to an overall increase during the normal production of collagen. No differences were found in the urinary excretion of pyridinium crosslinks between osteoporotic and normal women. However, this may indicate that the rate of bone resorption was similar between the two groups, and is not a measure of the actual pyridinium amounts of bone.

Köwitz et al. (58) studied osteoporotic, osteoarthritic, and normal women, and compared lysyl and prolyl hydroxylation between the three groups. They found an over hydroxylation of lysine in both alpha sub-units in the cortical and trabecular bone in the neck region of the human femur, and in trabecular bone in the femur head in the 
osteoporotic group. Lysyl hydroxylation was similar between the osteoarthritic and normal group. Prolyl hydroxylation was found to be similar in all compartments of bone between all three subgroups. Over modification of lysine in collagen is believed to occur during the repair of fractures (5). Köwitz, however, took bone within two days of fracture to eliminate any increase due to fracture healing. Because of this, they believe the observed increase in lysine hydroxylation precedes osteoporosis and is not a consequence of the disease. Whether the increase in hydroxylation causes changes in the formation of mature crosslinks is unknown. It is known that bone and cartilage contain a higher number of hydroxylysine residues in the telopeptide regions of collagen than does the collagen of skin. This predisposes the formation of pyridinium crosslinks in bone. In skin, lysyl oxidase acts more on lysine residues in the telopeptide regions, resulting in the formation of histidine crosslinks (57). Perhaps the increase in hydroxylation changes the ratio of pyridinoline to deoxypyridinoline in bone or the ratio of pyridinium to collagen. Another possibility is that the increased hydroxylation changes the amount of glycosylation to lysine residues in collagen, which in turn may effect collagen fiber formation, crosslinking, and/or mineralization.

Pyridinium crosslinks are known to be important for correct bone formation from several studies on Ehler Danlos Syndrome and lathyrism. These studies have shown that proper crosslinking is an important determinant of bone strength. Why pyridinium content predicts fracture resistance in men is unknown. Though not confirmed in this study, the possibility that males may have less pyridinium crosslinks than females can be raised. Also the ratio of pyridinium to collagen may have importance to bone strength. Perhaps these mature crosslinks only play a role in strengthening bone when they are 
present in a limited amount. This possibility may warrant continued research of this type, although this is not a finding seen in other studies $(76,77,78)$.

In conclusion, type-I collagen content appears to be a determinant of bone strength, specifically in tension fracture resistance. Collagen content may decrease with patient age, though this was not confirmed. Also shown in this study is that collagen may have gender-specific differences. These may include fractional composition of bone and the ratio of pyridinium to collagen, which may relate to bone strength. Others have shown that not only is the quantity of collagen important, but the quality of the protein also contributes to bone strength. The biochemical changes in collagen as age increases include increased hydroxylation of lysine, increased glycosylation of the sub-units, and a decrease in reducible divalent crosslinks. How these parameters may effect the mature trivalent pyridinium crosslinks remains unknown. This study also suggests that the amount of pyridinium crosslinks may have specific gender differences, and this may relate to the age of the donor and to bone strength. 


\section{References}

1. Kanis, J.A., Melton, III, L.J., Christiansen, C., Johnston, C.C., Khaltaev, N. The Diagnosis of Osteoporosis. J. Bone Miner. Res. 9-8 pp 1137-1141, 1994.

2. Parisien, M., Cosman, F., Mellish, R.W.E., Schnitzer, M., Nieves, J., Silverberg, S.J., Shane, E., Kimmel, D., Recker, R.R., Bilezikian, J.P., Lindsay, R., Dempster, D.W. Bone Structure in Postmenopausal Hyperthyroid, Osteoporotic, and Normal Women. J. Bone Miner. Res. 10-9 pp 1393-1399, 1995.

3. Marcus, R.: 'Organizational and Functional Aspects of Skeletal Health' in Osteoporosis (ed. Marcus), Blackwell Scientific Publications, Boston, Mass. pp 116, 1994.

4. Dempster, David W. The Contribution of Trabecular Architecture to Cancellous Bone Quality. J. Bone Miner. Res. 15-1 pp.20-23, 2000.

5. Lo Cascio, V., Bertoldo, F., Gambaro, G., Gasperi, E., Furlan, F., Colapietro, F., Lo Cascio, C., Campagnola, M., Urinary Galactosyl-Hydroxylysine in Postmenopausal Osteoporotic Women: A Potential Marker For Bone Fragility. J. Bone Miner. Res. 14-8 pp. 1420-1424, 1999.

6. Melton, L.J., Chrischilles, E.A., Cooper, C., Lane, A.W., Riggs, B.L. How Many Women Have Osteoporosis. J. Bone Miner. Res. 7-9 pp 1005-1010, 1992.

7. Aerssens, J., Van Audekercke, R., Geusens, P., Schot, L.P.C., Osman, A., Dequeker, J. Mechanical Properties, Bone Mineral Content, and Bone Composition (Collagen, Osteocalcin, IGF-I) of the Rat Femur: Influence of ovariectomy and Nondrolone Decanoate (Anabolic Steroid) Treatment. Calcif. Tissue Int. 53 pp. 269-277, 1993.

8. Kimmel, D. B., Recker, R. R.: 'Clinical Assessment of Bone Strength' in Osteoporosis (ed. Marcus), Blackwell Scientific Publications, Boston, Mass. pp 49-68, 1994.

9. Bell, K. L., Loveridge, N., Power, J., Garrahan, N., Stanton, M., Lunt, M., Meggitt, B. F., Reeve, J.: Structure of the Femoral Neck in Hip Fracture: Cortical Bone Loss in the Inferoanterior to Superoposterior Axis. J. Bone Miner. Res. 14-1 pp. 111-119, 1999.

10. Puustjärvi, K., Nieminen, J., Räsänen, T., Hyttinen, M., Helminen, H. J., Kröger, H., Huuskonen, J., Alhava, E., Kovanen, V.: Do More Highly Organized Collagen Fibrils Increase Bone Mechanical Strength in Loss of Mineral Density After One-Year Running Training? J. Bone Miner. Res. 14-3 pp. 321-329, 1999. 
11. Boskey, A. L., Wright, T. M., Blank, R. D.: Collagen and Bone Strength. J. Bone Miner. Res. 14-3 pp. 330-335, 1999.

12. Uzawa, K., Grzesik, W. J., Nishiura, T., Kuznetsov, S. A., Gehron Robey, P., Brenner, D. A., Yamauchi, M.: Differential Expression of Human Lysyl Hydroxylase Genes, Lysine Hydroxylation, and Cross-linking of Type I Collagen During Osteoblastic Differentiation In Vitro. J. Bone Miner. Res. 14-8 pp. 12721280, 1999.

13. Ciarelli, T. E., Fyhrie, D. P., Schaffler, M. B., Goldstein, S. A.: Variations in Three-Dimensional Cancellous Bone Architecture of the Proximal Femur in Female Hip Fractures and in Controls. J. Bone Moner. Res. 15-1 pp. 32-40, 2000.

14. Riggs, B. L.: Overview of Osteoporosis. Western Journal Med. 154 pp 63-77, 1991.

15. Arnaud, C.D. : Osteoporosis: Using "Bone Markers" for Diagnosis and Monitoring. Geriatrics 53 pp 24-30, 1996.

16. Garnero, P., Hausherr, E., Chapuy, M.-C., Marcelli, C., Grandjean, H., Muller, C., Cormier, C., Bréart, G., Meunier, P.J., Delmas, P.D.: Markers of Bone Resorption Predict Hip Fracture in Elderly Women: The EPIDOS Prospective Study. J. Bone Miner. Res. 11-10 pp 1531-1538, 1996.

17. Kohrt, W.M., Snead, D.B., Slatopolsky, E., Birge, Jr., S.J. : Additive Effects of Weight-Bearing Exercise and Estrogen on Bone Mineral Density in Older Women. J. Bone Miner. Res. 10-9 pp 1303-1311, 1995.

18. Mundy, G. R. : 'Bone Resorbing Cells' in Primer on Metabolic Bone Diseases and Disorders of Mineral Metabolism : 2nd Edition (ed. Fauvus), Am. Soc. Bone Miner. Res., Kelseyville, CA. pp 25-32, 1993.

19. Price, C.P. : Case Studies in Metabolic Bone Disease: Assessing the Potential for Biochemical Markers. LabMedica International 8-1, 1996.

20. Roodman, G.D. : Advances in Bone Biology: The Osteoclast. Endo Rev. 17-4 pp 308-332, 1996.

21. Jilka, R. L., Manolagas, S. C.: 'The Cellular and Biochemical Basis of Bone Remodeling' in Osteoporosis (ed. Marcus), Blackwell Scientific Publications, Boston, Mass. pp 17-47, 1994.

22. Öz, O. K., Zerwekh, J. E., Fisher, C., Graves, K., Nanu, L., Millsaps, R., Simpson, E. R.: Bone Has a Sexually Dimorphic Response To Aromatase Deficiency. J. Bone Miner. Res. 15-3 pp. 507-514, 2000. 
23. Faulkner, K. G.: Bone Matters: Are Density Increases Necessary to Reduce Fracture Risk? J. Bone Miner. Res. 15-2 pp. 183-187, 2000.

24. Bätge, B., Diebold, J., Stein, H., Bodo, M., Müller, P. K.: Compositional Analysis of the Collagenous Bone Matrix. A Study on Adult Normal and Osteopenic Bone Tissue. Euro. J. Clin. Invest. 22 pp. 805-812, 1992.

25. Melton III, L. J., Atkinson, E. J., O'Connor, M. K., O'Fallon, W. M., Riggs, L. B.: Determinates of Bone Loss from the Femoral Neck in Women of Different Ages. J. Bone Miner. Res. 15-1 pp. 24-31, 2000.

26. Calvo, M.S., Eyre, D.R., Gundberg, C.M. : Molecular Basis and Clinical Application of Biological Markers of Bone Turnover. Endocr. Rev. 17-4 pp. 333-368, 1996.

27. Baron, R. : 'Anatomy and Ultrastructure of Bone' in Primer on Metabolic Bone Diseases and Disorders of Mineral Metabolism : 2nd Edition (ed. Fauvus), Am. Soc. Bone Miner. Res., Kelseyville, CA. pp 3-9, 1993.

28. Landis, W. J.: The Strength of a Calcified Tissue Depends in Part on the Molecular Structure and Organization of its Constituent Mineral Crystals in their Organic Matrix. Bone 16-5 pp. 533-544, 1995.

29. Ingram, R.T., Park, Y.K., Clarke, B.L., Fitzpatrick, L.A. : Age- and Gender-Related Changes in the Distribution of Osteocalcin in the Extracellular Matrix of Normal Male and Female Bone. J. Clin. Invest. 93 pp 989-997, 1994.

30. Puzas. J.E. : 'The Osteoblast' in Primer on Metabolic Bone Diseases and Disorders of Mineral Metabolism : 2nd Edition (ed. Fauvus), Am. Soc. Bone Miner. Res., Kelseyville, CA. pp 15-21, 1993.

31. Termine, J.D. : 'Bone Matrix Proteins and the Mineralization Process' in Primer on Metabolic Bone Diseases and Disorders of Mineral Metabolism : 2nd Edition (ed. Fauvus), Am. Soc. Bone Miner. Res., Kelseyville, CA. pp 21-25, 1993.

32. Wright, G. M., Leblond, C. P. : Immunohistochemical Localization of Procollagens. J. Histochem. Cytochem. 29-7 pp 791-804, 1981.

33. Burgeson, R.E., Nimni, M.E. : Collagen Types : Molecular Structure and Tissue Distribution. Clin. Ortho. Related Res. 282 pp 250-271, 1992.

34. Hoshi, K., Kemmotsu, S., Takeuchi, Y., Amizuka, N., Ozawa, H.: The Primary Calcification of Bone Follows Removal of Decorin and Fusion of Collagen Fibrils. J. Bone Miner. Res. 14-2 pp. 273-280, 1999. 
35. Canalis, E. : 'Regulation of Bone Remodeling' in Primer on Metabolic Bone Diseases and Disorders of Mineral Metabolism : 2nd Edition (ed. Fauvus), Am. Soc. Bone Miner. Res., Kelseyville, CA. pp 33-37, 1993.

36. Prockop, D. J., Kivirikko, K. I.: Heritable Diseases of Collagen. New England J. Med. 311-6 pp 376-385, 1984.

37. Cheah, K. S. E.: Collagen Genes and Inherited Connective Tissue Disease. Biochem. J. 229 pp 287-303, 1985.

38. Prockop, D.J., Kadler, K.E., Hojima, Y., Constantinou, C.D., Dombrowski, K.E., Kuivaniemi, H., Tromp, G., Vogel, B. : Expression of Type I Procollagen Genes. Ciba Foundation Symposium 136, pp 142- 160, 1988.

39. Teitelbaum, S. L. : 'Molecular Defects in Bone Developement' in Primer on Metabolic Bone Diseases and Disorders of Mineral Metabolism : 2nd Edition (ed. Fauvus), Am. Soc. Bone Miner. Res., Kelseyville, CA. pp 10-15, 1993.

40. Bonadio, J., Goldstein, S.A.: 'Our Understanding of Inherited Skeletal Fragility and What this has Taught us about Bone Structure and Function' in Cellular and Molecular Biology of Bone (ed. Noda),

41. Prockop, D. J.: Mutations That Alter the Primary Structure of Type I Collagen. J. Biol. Chem. 265-26 pp. 15349-15352, 1990.

42. Vetter, U., Weis, M. A., Mörike, M., Eanes, E. D., Eyre, D. R.: Collagen Crosslinks and Mineral Crystallinity in Bone of Patients with Osteogenesis Imperfecta. J. Bone Miner. Res. 8-2 pp. 133-137, 1993.

43. Voet, D., Voet, J.G.: Biochemistry: 2nd Edition (ed. Ardwin), John Wiley and Sons, Inc. New York, N.Y. pp 156-163, 1007-1010, 1995.

44. Fraser, R.D.B., MacRae, T.P. Conformation in Fibrous Proteins and Related Synthetic Polypeptides Academic Press, New York, New York, 1973.

45. Vailas, A.C., Zernicke, R.F., Grindeland, R.E., Kaplansky, A., Durnova, G.N., Li, K-C., Martinez, D.A. : Effects of Spaceflight on Rat Humerous Geometry, Biomechanics, and Biochemistry. FASEB J. 4 pp 47-54, 1990.

46. Wenstrup, R. J., Murad, S., Pinnell, S. R.: Ehlers-Danlos Syndrome Type VI: Clinical Manifestations of Collagen Lysyl Hydroxylase Deficiency. J. Pedia. 115-3 pp. 405-409, 1989.

47. Prockop, D. J., Kuivaniemi, H.: Inborn Errors in Collagen. Rheumatology 10 pp 246-271, 1986. 
48. Bailey, A. J., Wotton, S. F., Thompson, P. W.: Post-Translational Modifications in the Collagen of Human Osteoporotic Femoral Head. Biochem. Biophys. Res. Comm. $185-3$ pp. 801-805, 1992.

49. Amudeswari, S., Liang, J. N., Chakrabarti, B.: Polar-Apolar Characteristics and Fibrillogenesis of Glycosylated Collagen. Collagen Rel. Res. 7 pp. 215-223, 1987.

50. Yamauchi, M., Katz, E. P., Otsubo, K., Teraoka, K., Mechanic, G. L.: CrossLinking and Stereospecific Structure of Collagen in Mineralized and Nonmineralized Skeletal Tissues. Connect. Tiss. Res. 21 pp. 159-169, 1989.

51. Hodge, A.J., Petruska, J.A., 'Recent Studies with the Electron Microscope on Ordered Aggregates of the Tropocollagen Macromolecule' in Aspects of Protein Structure. (ed. Ramachandran) Academic Press N.Y., N.Y. 1963.

52. Termine, J.D. : Non-Collagen Proteins in Bone. Ciba Foundation Symposium 136, pp 178-202, 1988.

53. Eastell, R., Mallinak, N., Weiss, S., Ettinger, M., Pettinger, M., Cain, D., Flessland, K., Chesnut III, C.: Biological Variability of Serum and Urinary NTelopeptides of Type-I Collagen in Postmenopausal Women. J. Bone Miner. Res. 15-3 pp. 594-598, 2000.

54. Colwell, A., Russel, G.G., Eastell, R. : Factors Affecting the Assay of Urinary 3Hydroxypyridinium Crosslinks of Collagen as Markers of Bone Resorption. Eur. J. Clin. Invest 23 pp 341-349, 1993.

55. Egger, C.D., Muhlbauer, R.F., Delmas, P.D., Marks, S.C., Fleisch, H. : Evaluation of Urinary Pyridinium Crosslink Excretion as a Marker of Bone Resorption in the Rat. J. Bone Miner. Res. 9-8 pp 1211-1219, 1994.

56. Eyre, D.R., Koob, T.J., Van Ness, K.P. : Quantification of Hydroxypyridinium Crosslinks in Collagen by High Performance Liquid Chromatography. Anal. Biochem. 137 pp 380-388, 1984.

57. Seibel, Markus J., Robins, Simon P., Bilezikian, John P.: Urinary Pyridinium Crosslinks of Collagen, Specific Markers of Bone Resorption in Metabolic Bone Disease. Trends Endocrinol. Metab. 3 pp 263-270, 1992.

58. Köwitz, J., Knippel, M., Schuhr, T., Mach, J.: Alteration in the Extent of Collagen I Hydroxylation, Isolated from Femoral Heads of Women with a Femoral Neck Fracture Caused by Osteoporosis. Calcif. Tissue Int. 60 pp. 501-505, 1997. 
59. Delmas, Pierre, D., Gineyts, Evelyne., Bertholin, Annick., Garnero, Patrick., Marchand, François.: Immunoassay of Pyridinoline Crosslink Excretion in Normal Adult and in Pagets Disease. J. Bone Miner. Res. 8 pp 643-648, 1993.

60. McPherson, J. M., Wallace, D. G., Sawamura, S. J., Conti, A., Condell, R. A., Wade, S., Piez, K.A.: Collagen Fibrillogenesis In Vitro : A Characterization of Fibril Quality as a Function of Assembly Conditions. Collagen Rel. Res. 5 pp. 119-135, 1985.

61. Gertz, B.J., Shao, P., Hanson, D.A., Quan, H., Harris, S.T., Genant, H.K., Chesnut, III, C.H., Eyre, D.R. : Monitoring Bone Resorption in Early Postmenopausal Women by an Immunoassay for Cross-Linked Collagen Peptides in Urine. J. Bone Miner. Res. 9-2 pp 135-142, 1994.

62. Kamel, S., Brazier, M., Neri, V., Picard, C., Samsom, L., Desmet, G., Sebert, J.L. Multiple Molecular Forms of Pyridinolines Cross-Links Excreted in Human Urine Evaluated by Chromatographic and Immunoassay Methods. J. Bone Miner. Res. 10-9 pp 1385-1392, 1995.

63. Robins, S.P., Black, D., Paterson, C. R., Reid, D. M., Duncan, A., Seibels, M. J.: Evaluation of Urinary Hydroxypyridinium Crosslink Measurements as Resorption Markers in Metabolic Bone Diseases. Euro. J. Clin. Invest. 21 pp 310-315, 1991.

64. Smith, J.W. Molecular Pattern of Native Collagen. Nature 219 pp 157-158, 1968.

65. Katz, E. P., Wachtel, E., Yamauchi, M., Mechanic, G. L.: The Structure of Mineralized Collagen Fibrils. Conn. Tis. Res. 21 pp. 149-158, 1989.

66. Vailas, A.C., Vanderby, R., Martinez, D.A., Ashman, R.B., Ulm, M.J., Grindeland, R.E., Durnova, G.N., Kaplansky, A. : Adaptations of Young Adult Rat Cortical Bone to 14 Days of Spaceflight. J. Appl. Physiol. 73(2), Suppl.: 4S9S, 1992.

67. Veis, A.: Phosphoproteins From Teeth and Bone. Ciba Foundation Symposium 136, pp161-177, 1988.

68. Vermeer, C., Jie, K-S. G., Knapen, M.H.J.: Role of Vitamin K in Bone Metabolism. Annu. Rev. Nutr. 15 pp 1-22, 1995.

69. Young, M. F., Ibaraki, K., Kerr, J. M., Heegaard, A.M.: 'Molecular and Cellular Biology of the Major Non-Collagenous Proteins in Bone' in Cellular and Molecular Biology of Bone (ed. Noda), Academic Press, Inc. San Diego, Ca. pp 191-234, 1993. 
70. Hodge, A. J.: Molecular Molecules Illustrating the Possible Distributions of 'Holes' in Simple Systematically Staggered Arrays of Type-I Collagen Molecules in Native-Type Fibrils. Conn. Tis. Res. 21 pp. 137-147, 1989.

71. Van Den Bos, T., Beertsen, W.: Bound Phosphoproteins Enhance Mineralization of Alkaline Phosphatase-Collagen Complexes In Vivo. J. Bone Miner. Res. 9-8 pp 1205-1209, 1994.

72. Yeni, Y.: Fracture Mechanics of Human Cortical Bone: The Relationship of Geometry, Microstructure and Composition with the Fracture of the Tibia, Femoral Shaft and the Femoral Neck. 1998.

73. Dunphy, M.J., Bhide, M. V., Smith, D. J.: Determination of Hydroxyproline in Tissue Collagen. J. Chromotog. 420 pp. 394-397, 1987.

74. Bidlingmeyer, B. A., Cohen, S. A., Tarvin, T. L.: Rapid Analysis of Amino Acids Using Precolumn Derivitization. J. Chromotog. 336 pp. 93-104, 1984.

75. Eyre, D.: New Biomarkers of Bone Resorption. J. Clin. Endo. \& Metab. 74 pp. 470A-B, 1992.

76. Bailey, A.J., Wotton, S.F., Sims, T.J., Thompson, P.W.: Biochemical Changes in the Collagen of Human Osteoporotic Bone Matrix. Connective Tissue Research, 29 pp. 119-132, 1993.

77. Oxlund, H., Mosekilde, Li, Ørtoft, G.: Reduced Concentration of Collagen Reducible Crosslinks in Human Trabecular Bone with Respect to Age and Osteoporosis. Bone, 19-5, pp. 479-484, 1996.

78. Bailey, A.J., Sims, T.J., Ebbesen, E.N., Mansell, J.P., Thompson, J.S., Mosekilde, Li.: Age-Related Changes in the Biochemical Properties of Human Cancellous Bone Collagen: Relationship to Bone Strength. Calcif. Tissue Int. 65, pp.203$210,1999$.

79. Holland, E.F. Nigel, Studd, John W.W., Mansell, Jason P., Leather, Andrew T., Bailey, Allen J.: Changes in Collagen Composition and Cross-links in Bone and Skin of Osteoporotic Postmenopausal Women Treated with Percutaneous Estradiol Implants. Obstetrics and Gynecology 83-2, pp. 180-183, 1994.

80. Sanada, H., Shikata, J., Hamamoto, H., Ueba, Y., Yamamuro, T., Takeda, T.: Changes in Collagen Cross-linking and Lysyl Oxidase by Estrogen. Biochim. et Biophys. Acta 541, pp. 408-413, 1978.

81. Ernst, M., Schmid, Ch., Froesch, E.R.: Enhanced Osteoblast Proliferation and Collagen Gene Expression by Estradiol. Proc. Natl. Acad. Sci. 85, pp. 23072310, 1988. 
82. Knott, L., Bailey, A.J.: Collagen Cross-links in Mineralizing Tissues: A Review of Their Chemistry, Function, and Clinical Relevance. Bone 22, pp. 181-187, 1998.

83. Danielsen, C.C., Mosekilde, L., Svenstrup, B.: Cortical Bone Mass, Composition, and Mechanical Properties in Female Rats in Relation to Age, Long-term Ovariectomy, and Estrogen Substitution. Calcif. Tissue Int. 52, pp. 26-33, 1993.

84. Lian, J.B., Stein, G.S., Canalis, E., Robey, P.G., Boskey, A.L.: 'Bone Formation: Osteoblast Lineage Cell, Growth Factors, Matrix Proteins, and the Mineralization Process' in Primer on Metabolic Bone Diseases and Disorders of Mineral Metabolism: 4th Edition (ed. Fauvus), Am. Soc. Bone Miner. Res., Kelseyville, CA. pp.14-29, 1999. 